\title{
WestVirginiaUniversity
}

THE RESEARCH REPOSITORY @ WVU

Graduate Theses, Dissertations, and Problem Reports

2017

\section{Effect of Chamber Pressure on the Quality of Breech Face and Firing Pin Impressions}

James A. Hamilton

Follow this and additional works at: https://researchrepository.wvu.edu/etd

\section{Recommended Citation}

Hamilton, James A., "Effect of Chamber Pressure on the Quality of Breech Face and Firing Pin Impressions" (2017). Graduate Theses, Dissertations, and Problem Reports. 5743.

https://researchrepository.wvu.edu/etd/5743

This Thesis is protected by copyright and/or related rights. It has been brought to you by the The Research Repository @ WVU with permission from the rights-holder(s). You are free to use this Thesis in any way that is permitted by the copyright and related rights legislation that applies to your use. For other uses you must obtain permission from the rights-holder(s) directly, unless additional rights are indicated by a Creative Commons license in the record and/ or on the work itself. This Thesis has been accepted for inclusion in WVU Graduate Theses, Dissertations, and Problem Reports collection by an authorized administrator of The Research Repository @ WVU. For more information, please contact researchrepository@mail.wvu.edu. 


\title{
Effect of Chamber Pressure on the Quality of Breech Face and Firing Pin Impressions
}

\author{
James A. Hamilton \\ Thesis submitted \\ to the Eberly College of Arts and Sciences \\ at West Virginia University \\ in partial fulfillment of the requirements for the degree of \\ Master of Science in \\ Forensic \& Investigative Science \\ Dr. Keith Morris, Ph.D., Chair \\ Dr. Afzel Noore, Ph.D. \\ Mr. Dwight Deskins, B.S. \\ Department of Forensic \& Investigative Science \\ Morgantown, West Virginia \\ 2017
}

Keywords: IBIS, Match Scores, Firing Pin, Breech Face, Chamber Pressure Copyright 2017 James A. Hamilton 


\title{
ABSTRACT \\ Effect of Chamber Pressure on the Quality of Breech Face and Firing Pin Impressions
}

\author{
James A. Hamilton
}

Firearms evidence plays a vital role in shooting incident investigations. Included in this type of evidence is the comparison of fired cartridge casings. The evaluation of cartridge casings can inform investigators as to the number of firearms used in an incident. This evaluation includes the observation of breech face impressions imparted on the cartridge casing by the firearm. The comparison of these markings can assist investigators in the apprehension of perpetrators. However, the quality of these impressions may vary based on the pressures generated within a single system during firing leading to the appearance of multiple firearms being involved. Investigation into the differences in breech face markings generated by a single firearm should be conducted.

In this study, .38 Special and .357 Magnum ammunition was reloaded to ensure variables were kept constant between each cartridge while varying the powder type and weight. Pressures generated during firing were recorded using the PressureTrace II system and muzzle velocities were measured using a MagnetoSpeed ballistic chronograph. The PressureTrace II system allowed for plots of pressure against time to be generated for each firing repetition. Each fired cartridge case was then entered into the Integrated Ballistic Identification System (IBIS) allowing breech face and firing pin correlation scores between the cartridge cases to be generated. Correlation scores between the cartridge cases were then assessed for differences in breech face and firing pin scores. It was found that cartridges with greater differences between their charge weights and pressure gave lower average breech face scores while cartridges with more similar charge weights and pressures gave higher average breech face scores. However, firing pin scores showed less variation based on these variables. 


\section{Contents}

1 Introduction 1

1.1 Value of Breech Face Markings . . . . . . . . . . . . . . . . . . . 1

2 Literature Review 3

2.1 Factors Affecting Chamber Pressure . . . . . . . . . . . . . . . . 3

2.2 Volume Inside the Casing . . . . . . . . . . . . . . . . . . . . . 3

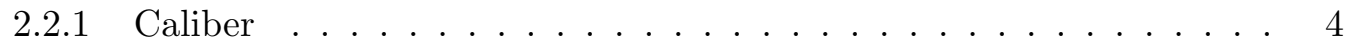

2.2 .2 Seating Depth . . . . . . . . . . . . . . . . . 4

2.3 Diameter, Weight, and Neck Tension of the Projectile . . . . . . . . 4

2.4 Powder Temperature . . . . . . . . . . . . . . . . . 5

2.5 Powder Characteristics . . . . . . . . . . . . . . . . . . 5

2.6 Position of Powder in the Chamber . . . . . . . . . . . . . . . . 8

2.7 Calculating Breech Pressure . . . . . . . . . . . . . . . . 8

2.8 Measuring Chamber Pressure . . . . . . . . . . . . . . . . . . 9

2.8.1 Copper Crusher Gauge. . . . . . . . . . . . . . . . . . . . . 9

2.8.2 Piezoelectric Gauge . . . . . . . . . . . . . . . . . . . . . 10

2.8.3 Strain Gauge . . . . . . . . . . . . . . . . . . . . . 11

2.9 Measuring Projectile Velocity . . . . . . . . . . . . . . . . . . . 12

2.10 Theoretical Evaluation of Internal Ballistics . . . . . . . . . . . . . 13

$2.11 \mathrm{IBIS}^{\circledR} \ldots \ldots \ldots \ldots \ldots \ldots \ldots \ldots \ldots \ldots$

2.12 Pressure Trace $\mathrm{II}^{\mathrm{TM}} \ldots \ldots \ldots \ldots \ldots \ldots \ldots \ldots \ldots$

2.13 Quickload $^{\mathrm{TM}} \ldots \ldots \ldots \ldots \ldots \ldots \ldots \ldots$

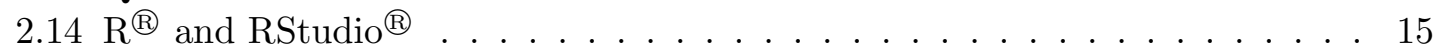

$\begin{array}{llr}3 & \text { Studies } & 16\end{array}$

4 Methods $\quad 18$

$4.1 \mathrm{R}^{\circledR}$ Script Development . . . . . . . . . . . . . . . . . . . . . 18

4.2 Ammunition and Reloading . . . . . . . . . . . . . . . . . 20

4.3 Firing and Data Collection . . . . . . . . . . . . . . . 21

4.4 IBIS ${ }^{\circledR}$ Data Collection . . . . . . . . . . . . . . . . . . . . . . . 21

4.5 Pressure Trace $\mathrm{II}^{\mathrm{TM}} \ldots \ldots \ldots \ldots \ldots \ldots \ldots . \ldots \ldots \ldots$ 
4.6 MagnetoSpeed $^{\mathrm{TM}}$ Data Collection ................... . . 24

4.7 Data Analysis . . . . . . . . . . . . . . . . . . . . 24

5 Results and Discussion $\quad 25$

6 Conclusions $\quad 41$

7 Appendix A: Pressure Trace Comparisons 44

8 Appendix B: R Scripts $\quad 56$

8.1 Handbook on Weaponry Internal Ballistics Estimation . . . . . . . . . . 56

8.2 Plot Exported Quickload ${ }^{\mathrm{TM}}$ Data . . . . . . . . . . . . . . . . 60

8.3 Process Exported IBIS ${ }^{\circledR}$ data . . . . . . . . . . . . . . . . . . . . 62

8.4 Process Pressure Trace $\mathrm{II}^{\mathrm{TM}}$ Shot Data . . . . . . . . . . . . . . . 76

8.5 Adjust Pressure Trace $\mathrm{II}^{\mathrm{TM}}$ Trace Data . . . . . . . . . . . . . . . . . 80

8.6 Compile Pressure Trace $\mathrm{II}^{\mathrm{TM}}$, Quickload ${ }^{\mathrm{TM}}$, and R Script Traces . . . . . . 81

8.7 Evaluation of $\mathrm{IBIS}^{\circledR}$ Data . . . . . . . . . . . . . . . . . . 83 


\section{Introduction}

\subsection{Value of Breech Face Markings}

Breech face markings vary between each firearm based upon the manufacturing process. The process by which the breech face is manufactured can vary between manufacturers and can be done by filing or rotary milling which will create linear striation marks or concentric circular markings respectively. The markings vary within the same method as well based upon the amount of filing, polishing, and application of finish to the breech (1). During the firing of a firearm, the pressure build-up inside the chamber works on the base of the bullet to force it down the barrel, but also on the inner walls of the cartridge casing, forcing it against the walls of the chamber as well as forcing the primer out of its pocket and against the breech face. This results in the creation of breech face markings on the primer and head stamp area of the cartridge casing $(2)$.

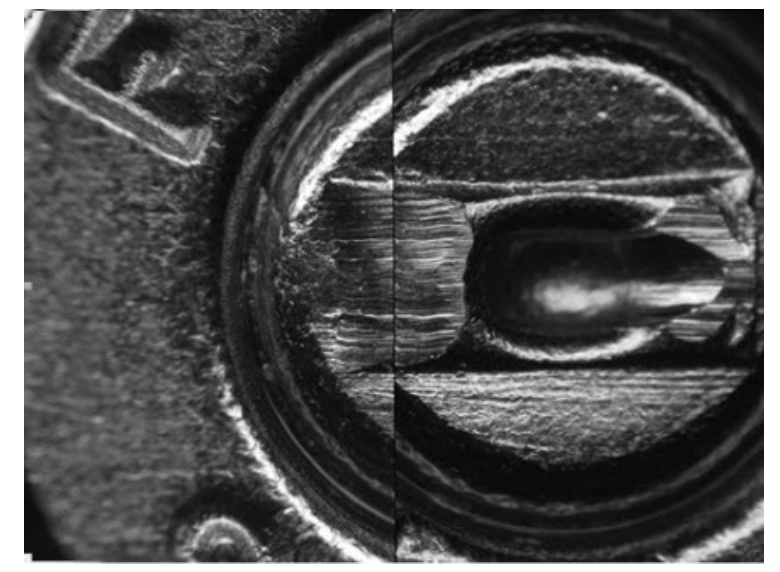

Figure 1.1: Image depicting the comparison of two fired cartridge casings using a comparison microscope.(3).

An example of a comparison of markings on the primer of a firearm can be seen in Figure 1.1. Variations in these markings can occur predominantly due to wear imparted on the breech from use over time. However, breech face markings are less vulnerable to this 
depreciation, which makes their comparison a reliable method for forensic identification purposes (1). A second possible source of variation in markings is the force with which the primer is forced against the breech face. This is dependent upon the chamber pressure generated during the firing process. Lower chamber pressures are less likely to develop similar breech face impressions repeatedly leading to the possible inability of an analyst to identify that two cartridges were fired from the same firearm (4).

This thesis assesses the effect on conclusions drawn during the comparison of two fired cartridge casings originating from the same firearm based on the differences in breech face markings due to variances in chamber pressure. This will assist investigators in determining a possible set of firearms used in an investigation as well as show whether incorrect conclusions may be drawn by using incorrect ammunition types when comparing test fires to cartridges collected at a scene depending on differences in pressure between them. Additionally, if various types of ammunition were used in separate shooting incidents utilizing the same firearm, this thesis may show that, although the breech face markings may seem sufficiently dissimilar to conclude separate firearms were used, the same firearm was used in multiple incidents. 


\section{Literature Review}

\subsection{Factors Affecting Chamber Pressure}

Variances in chamber pressure can be attributed to a multitude of factors. Bullet characteristics, powder load, bullet seating depth, position of powder before firing, powder temperature, powder characteristics, casing volume, and headspace are all variables that may affect the chamber pressure during firing. In order to determine chamber pressure, measurements are generally taken from the breech of the firearm, particularly when examining the propellants burning characteristics (5). This pressure can be manipulated by changing the aforementioned variables.

\subsection{Volume Inside the Casing}

The pressure inside a closed system filled with gas is dependent upon the number of collisions of the molecules of that gas with the walls of the system. The more constrained the molecules are within the system, the higher the number of collisions will occur leading to higher pressures (5). This constraint can be affected by the volume of empty space inside the casing. The volume inside a cartridge casing can be affected by multiple variables and the primary concern for the volume is the area in which the evolving gases have to expand. The seating depth of the bullet and load density both play role in determining how much open space is available for the gases to expand. The more volume the gases have to expand in, the lower the pressure will be and vice versa. Since the gases developed during the firing of a firearm are not ideal gases, the Nobel-Abel equation of state is best used due to its ability to account for the physical size of the gas molecules and the intermolecular forces created by their position in relation to one another. This equation can be stated as:

$$
p\left(V-m_{g} b\right)=m_{g} R T
$$

where $p$ is the pressure of the gas, $V$ is the volume the gas occupies, $m_{g}$ is the mass of the gas, $R$ is the specific gas constant, $T$ is the absolute temperature, and $b$ is the covolume of the gas which accounts for the size of the gas molecules and their interactions with one another (5). This equation shows how the volume affects the theoretical pressure and, after solving for $\mathrm{p}$, results in the equation: 


$$
p=\frac{m_{g} R T}{\left(V-m_{g} b\right)}
$$

Equation 2.2 shows the inverse relationship between the pressure developed inside the casing and the volume the gas occupies. This volume is affected by other factors as well.

\subsubsection{Caliber}

As the caliber of a firearm increases, the amount of powder, and pressure, needed to project the bullet down the barrel will increase leading to the need for larger casings. As the casings increase in size, so does the internal volume. However, the load density of the powder within the casing may vary. Load density can be described as the ratio of empty case volume to volume of the casing that the powder fills. The less space the powder fills, the larger the ratio becomes leading to a slower initial rate of combustion of the propellant (6). The second factor that affects the volume inside the casing is the seating depth of the bullet.

\subsubsection{Seating Depth}

The seating depth of the bullet can affect the pressure in two ways, the first of which is by affecting the volume inside the casing. By seating the bullet shallower, the volume inside the casing increases leading to a decrease in pressure generated during firing. The opposite applies when seating the bullet deeper. Deep bullet seating depths result in decreased cartridge case volume and higher pressures (7). The second way in which the seating depth of the bullet affects pressure is through the amount of friction required for it to begin its travel. When seated deeper, the bullet usually rests off of the lands of the barrel resulting in the neck tension being the only mechanical friction being imposed upon it. When it is seated shallower, to the extent that it contacts the lands of the barrel, it now has to overcome the neck tension as well as the static friction imposed upon it by the lands. This extra friction requires the development of higher pressures in order to unseat the bullet and project it down the barrel (8).

\subsection{Diameter, Weight, and Neck Tension of the Projectile}

Projectile characteristics can also play a role in the amount of pressure generated during firing. The weight and diameter of the bullet as well as the neck tension placed upon it by the cartridge can vary how much pressure must be generated in order for it to be projected down the barrel (9). This relationship between force and caliber can be shown by the formula:

$$
F=P A
$$

where $F$ is the force, $P$ is the pressure, and $A$ is the area upon which the pressure is exerted. This area is a function of the caliber (10). The weight of the projectile will also 
play a role in the pressure generated. The heavier the weight of the projectile, the more pressure needed to project it down the barrel. Neck tension placed on the projectile by the casing will also vary the pressure needed to project it down the barrel. As the neck tension increases, the amount of static friction increases requiring more pressure to unseat the bullet from the casing. This leads to an increase in pressure in the chamber (11).

\subsection{Powder Temperature}

The temperature of the powder before firing has been found to affect the muzzle velocity and ultimately pressure inside a firearm. For any given powder, the higher the temperature of the powder before firing, the higher the muzzle velocity (12). The relationship of the muzzle velocity to pressure via Newton's laws of motion, it can be ascertained that the pressure inside the chamber will also increase with temperature. The relationship between muzzle velocity and pressure can be ascertained by utilizing Newton's second law which can be written as:

$$
F=m a
$$

Where $F$ is the force, $m$ is the mass of the object, and $a$ is acceleration of the object (13). This can then be used in the equation:

$$
v=\frac{F t}{m}
$$

where $v$ is the velocity, $F$ is the force, $t$ is the time during which the force acts, and $\mathrm{m}$ is the mass of the projectile (6). The force in this equation originates from the pressure developed during the evolution of expanding gases in the chamber. The maximum force exerted on the base of the projectile can be determined by using Equation 2.6 above.

Figure 2.1 shows the relationship between the temperature of the powder and the resulting muzzle velocity.

\subsection{Powder Characteristics}

The powders used in modern cartridge casings have multiple characteristics, most of which affect the burn rate of the powder. The burn rate, in turn, affects the pressure generated inside the chamber. These characteristics include: grain shape, size, burning time, burning action, coatings, additives, and the type of powder.

The combustion of the powder is dependent upon its size and shape. The rate at which gases are evolved depends on the surface area of the powder (5). However, the surface area can also be dependent upon the shape which also plays a role in the type of burning the powder undergoes. If the powder grains contain a central core, it usually undergoes neutral burning, if it is cylindrical, spherical, or cubical in shape, it is commonly regressive burning, and if it has multiple perforations within it, it is usually progressive burning 


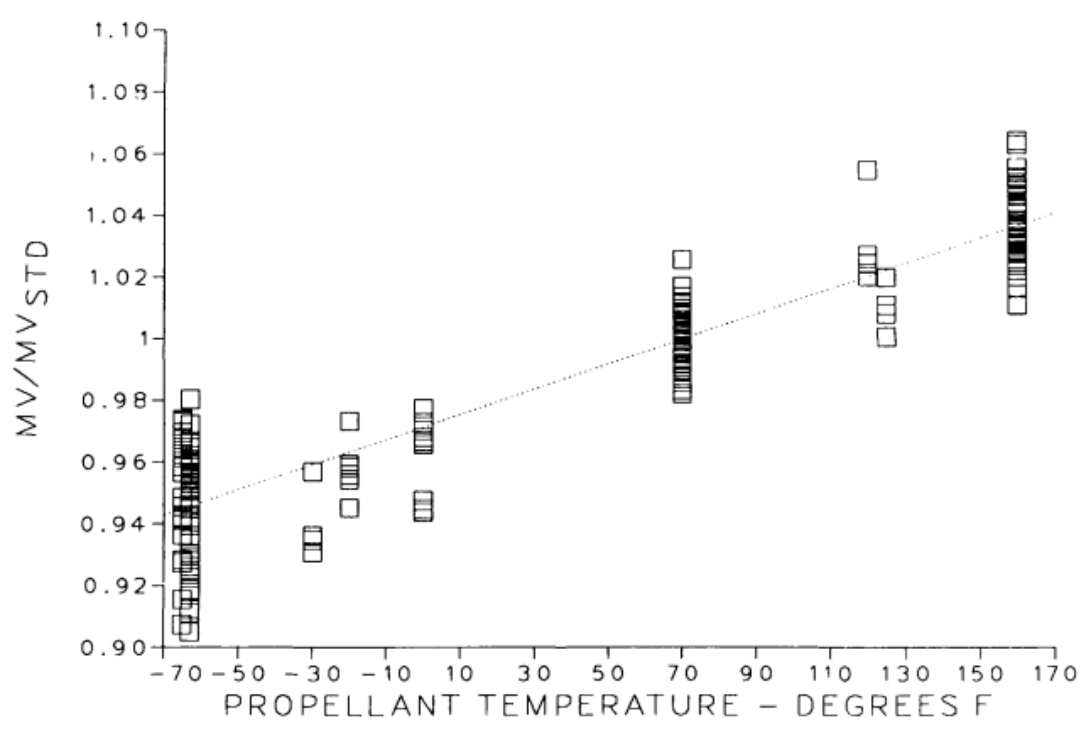

Figure 2.1: Graph showing the relationship between propellant temperature in degrees Fahrenheit with muzzle velocity propellant temperature coefficient. (12).

(14). Regressive burning refers to powders whose surface area decreases as combustion continues, neutral burning refers to powders whose outer surface are decreases while its inner surface area increases during combustion resulting in the net burning surface area remaining the same, and progressive burning refers to powders whose surface area increases as combustion continues since it burns from both its inner and outer surfaces (15). One common characteristic of propellant combustion is that it proceeds in the form of layers with the burn front parallel to the surface of the propellant (16).

The burn rate, and in relation to it, the rate of evolution of the gas affects the pressure generated greatly. Fast burning powders combust quickly resulting in the rapid rise of pressure in the chamber over a short period of time giving the bullet little time to move before maximum pressures are reached. Slow burning powders combust more slowly giving the projectile more time to travel before maximum pressures are reached (17). Due to this, it is common for pressures and forces generated from slow burning powders to be less than those generated from fast burning powders inducing the need for larger powder charges for slow burning powders in order to maintain sufficient pressures. Powders with smaller grains tend to be fast burning powders and are utilized mainly in firearms with shorter barrels such as handguns whereas slower burning powders are more commonly used in rifle cartridges (18). These burn rates are also affected by the chemical composition of the powder and the pressure inside the chamber. 

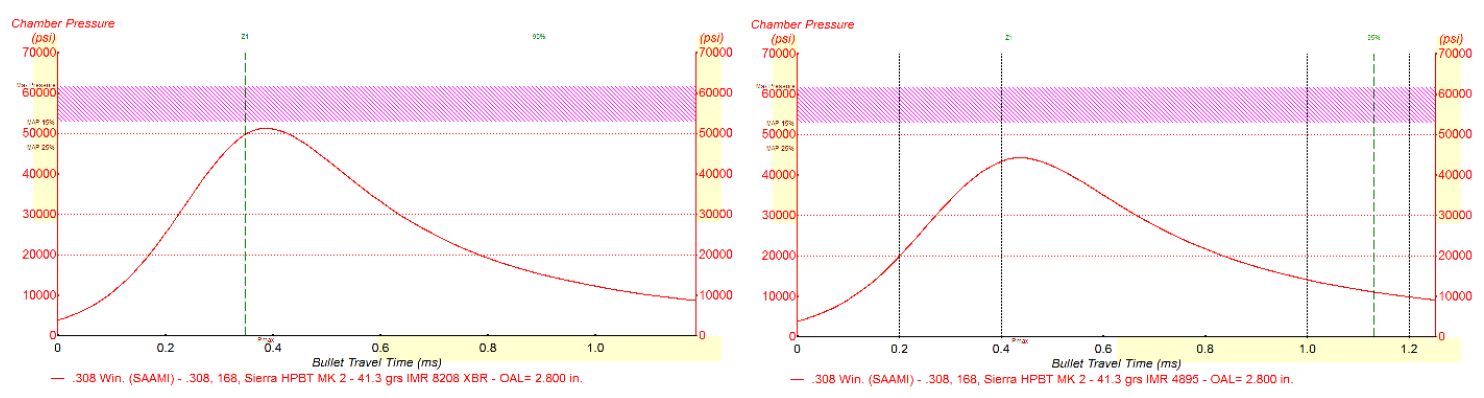

Figure 2.2: Illustration of the difference of pressure-time curves for fast (left) and slow (right) burning powders generated using QuickLOAD program

Figure 2.2 illustrates the difference in pressure-time curves for fast and slow burning powders with the fast burning powder on the left and the slow burning on the right. It can be seen that the slow burning powder has a broader curve with higher pressure at the muzzle where the fast burning powder has a steeper slope both before and after maximum pressure with lower pressure at the muzzle.

The combustion of the powder is dependent upon the pressure inside the chamber during the burning process and, as the pressure rises, the burn rate increases (5). During the burning process, solid propellant is being converted to gas resulting in an increase in volume inside the casing resulting in slight decreases in pressure. However, the evolving gases are developing into a closed environment resulting in an increase in pressure. This relationship can be written as:

$$
D \frac{d f}{d t}=-\beta p_{B}(t)
$$

where $D d f / d t$ is the burn rate, $\beta$ is a burn rate coefficient, and $p_{B}$ is the pressure at time $t(5)$. Separate from the pressure inside the chamber, the chemical composition of the powder also affects the burn rate.

Powders can be comprised of various components depending on the way they are meant to act. Most current day powders are either single or double base propellants. Single base propellants are comprised mostly of nitrocellulose and can be comprised of other additives that can help regulate the burning characteristics, amount of flash produced, or how stable the powder is. Diphenylamine is commonly added to powder to help stabilize it and coatings can be added to control burn rate (2). Coating the outside portion of the powder grains can result in more progressive burning powder. The coating is a slow burning substance but, as it burns off, the burn rate increases resulting in a more uniform pressure during the firing process (18). Single base propellants generally produce 1000 calories and 900 cubic centimeters of gas per grain and have a burn rate of .1 to 18 centimeters per second producing pressure up to 60,000 pounds per square inch (15). Another major 
ingredient that must be contained within the powder is fuel and an oxidizer. This is due to the lack of oxygen present in the casing to fuel combustion. Although there is a small amount present in the unfilled portion of the casing, it is often insufficient to fuel complete combustion which requires that the powder have its own fuel source (5). Double base powders are similar to single base powders with nitroglycerin added. This increases the potential energy of the powder as well as the stability. These powders are also more sensitive and can produce higher potential energy and heat as well as burn faster but can still be controlled with additives similar to single base propellants (15).

\subsection{Position of Powder in the Chamber}

The physical positioning of the powder in the chamber prior to firing can also affect the pressure generated. The more uniform the distribution of the powder within the chamber before firing, the more uniform the increase in chamber pressure will be (9). If the powder is located near the breech, the pressure will rise quickly near the breech and create a pressure wave which propagates in the direction of the base of the projectile. The same is true when the powder is located at the base of the projectile, the pressure wave will propagate toward the breech (9). This will result in the appearance of oscillations in the pressure as the pressure wave is rebounded off of the object it travels toward and returns to where it originated. In the case of the pressure wave originating at the breech, this results in higher breech pressures. In the case of the pressure wave originating at the base of the projectile, this results in faster projectile movement and lower mean chamber pressures as well as a reduced intensity in the pressure wave. This is due to the immediate rise in pressure at the base of the projectile. The area in which the pressure originates will have an immediate rise in pressure whereas the opposite location will have a slight delay in the increase in pressure (9). However, uniform distribution of powder results in a uniform rise in pressure throughout the chamber.

Figure 2.3 shows the phenomena previously described where the pressure waves generated can be observed as well as the lack of pressure waves in the instance of uniform distribution of propellant in the chamber.

\subsection{Calculating Breech Pressure}

Breech pressure, or backward thrust, can be calculated as the product of the area of the cartridge head and the chamber pressure (11). This allows for the calculation of the maximum breech pressure. In order to calculate breech pressures at various points in the firing process, concepts from fluid mechanics and the Lagrangian approach can be utilized. The Lagrangian approach includes the concept of the Lagrange gradient that states that a gradient of pressure exists within the system in which the pressure at the breech is always higher than the pressure at the base of the projectile (5). 


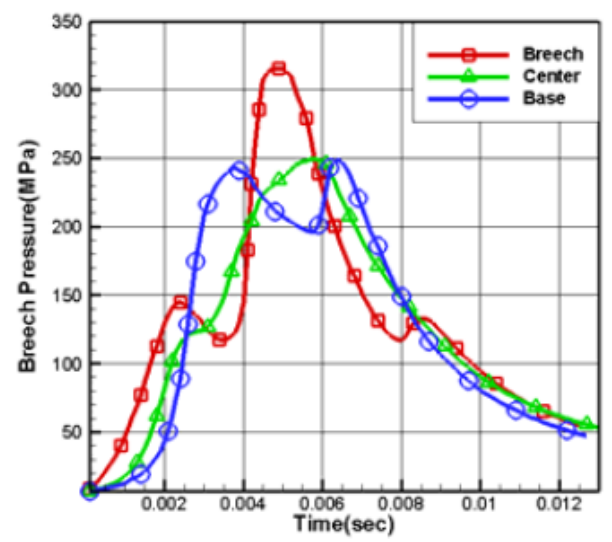

(a)

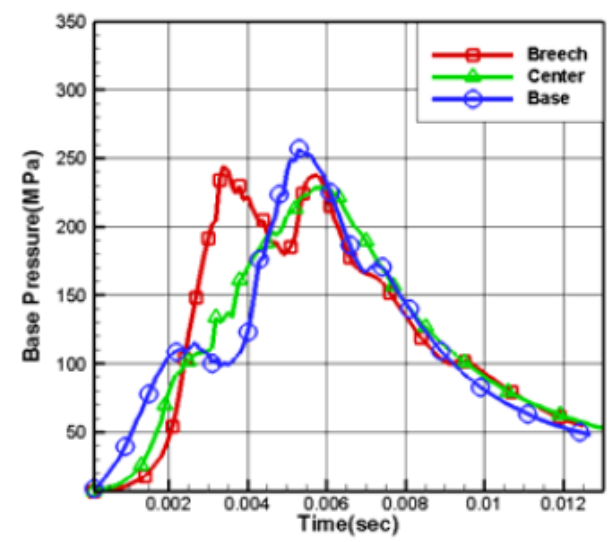

(b)

Figure 2.3: : Graphs showing the rise in breech and base pressure relative to propellant position in the cartridge casing before firing. Adapted from Jang 2011 (9).

\subsection{Measuring Chamber Pressure}

There are three ways of measuring the pressure generated during the firing of a firearm which come in two different forms: those that measure the maximum pressure only and those that measure the pressure as a function of time (15). Crusher gauges are of the first form with strain and piezoelectric gauges being the second.

\subsubsection{Copper Crusher Gauge}

The first method of pressure measurement is that of copper crusher gauges. This is the only form of pressure measurement that determines the maximum pressure rather than the pressure as a function of time. This method utilizes a gas check cap and steel piston that, when placed under pressure, compacts a copper or lead cylinder. The length of compression of the cylinder is then measured with a micrometer and the pressure is determined by reading a table relating the compression to the pressure that produces it (15). However, these pressures are produced by using a hydraulic press to crush cylinders at various pressures and must be corrected. Comparison to other pressure measurement techniques have shown that the results obtained by this measurement method should be multiplied by 1.2 to obtain a more accurate chamber pressure (15). 


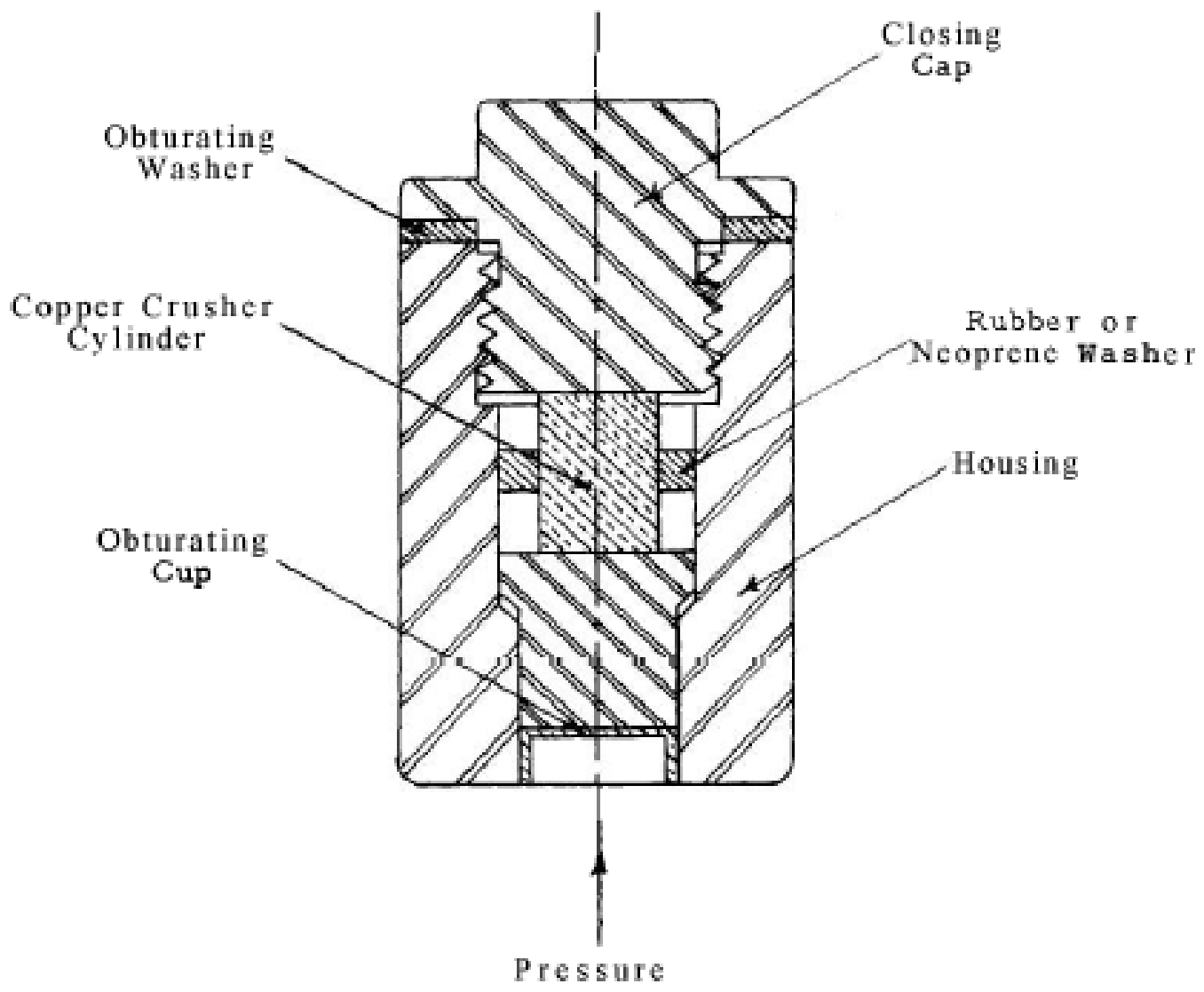

Figure 2.4: Image showing the internal construction of copper crusher gauge. (15).

\subsubsection{Piezoelectric Gauge}

The second method of measuring the pressure generated during firing is by use of a piezoelectric gauge. Piezoelectric gauges operate on the premise that certain types of crystals generate an electrical charge when placed under pressure. For this method, quartz crystals are the most common due to their availability and high breaking stress above alternative crystals (15). However, the response generated by quartz is dependent upon their structure. Due to this, this method is sensitive to the orientation of the crystals within the system and they require the pressure be applied via an anvil or piston type object (15). It is also possible to adjust the sensitivity of piezoelectric gauges by varying the number of crystals used. However, the crystals are subject to cracking if the force place upon them exceeds 13,000 pounds per square inch. The working pressure placed upon them can be adjusted by increasing the piston area which applies pressure to the crystals, but if the area is lowered below a given threshold, the piston can be deformed. This imposes 
a limit on the pressures that can be measured with the upper limit being 70,000 pounds per square inch and the lower limit being 15,000 pounds per square inch (15).

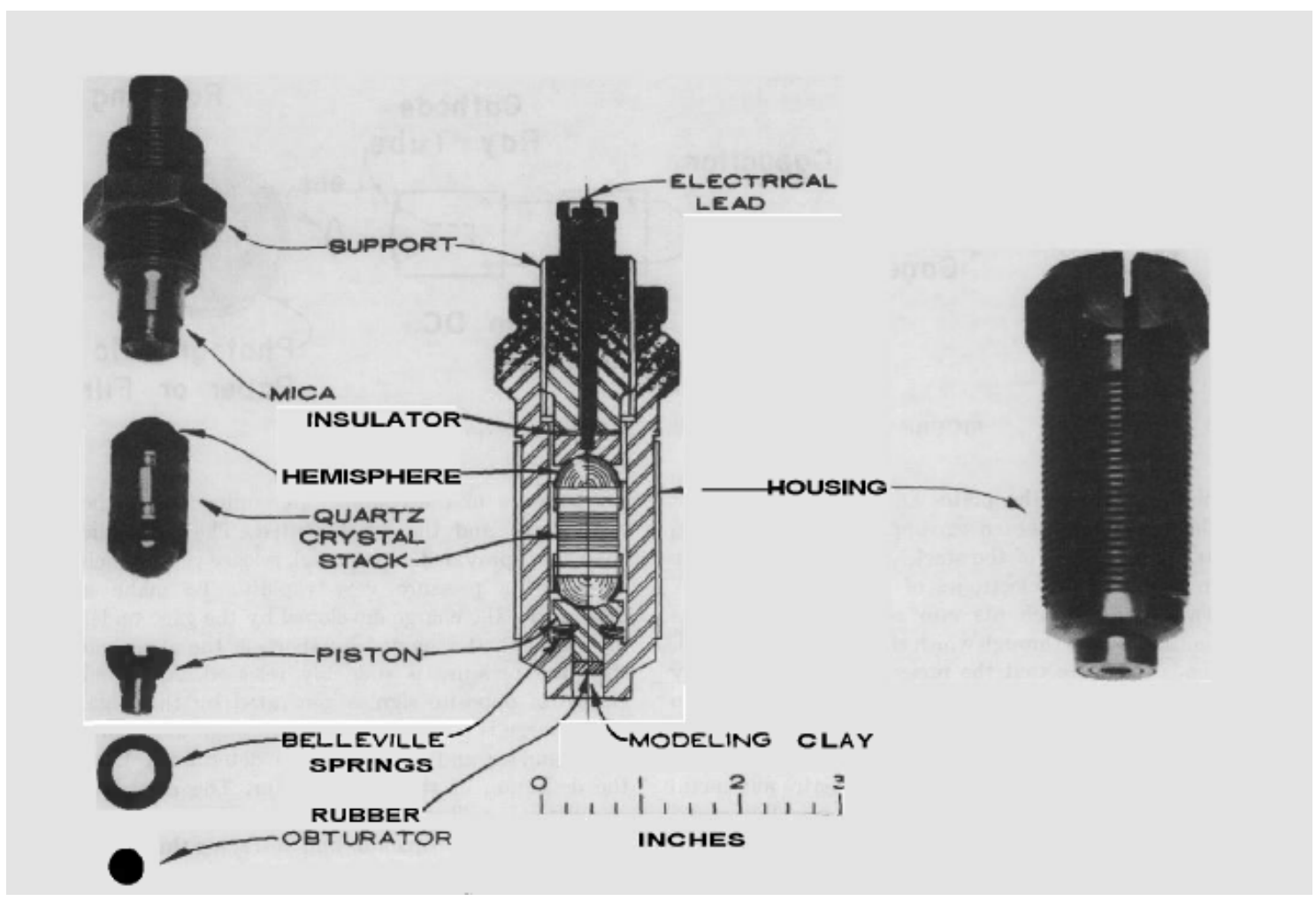

Figure 2.5: Image showing the construction of a Quartz Piezoelectric Gauge. (15).

\subsubsection{Strain Gauge}

The third type of pressure measurement system is that of strain gauges. Strain gauges operate by utilizing a short tube or ferrule on one end that will be subject to the pressure. A strain wire is wound around the ferrule and applied to the surface. This wire forms one part of a bridge circuit and, when pressure is applied, the bridge becomes unbalanced as a function of the pressure. This results in the development of an electromagnetic field which is fed to an amplifier and fed to a computer controller which records the data (15). The pressure range of these gauges can be varied by utilizing different ferrules and are inherently better at measuring lower pressures than piezoelectric gauges. 


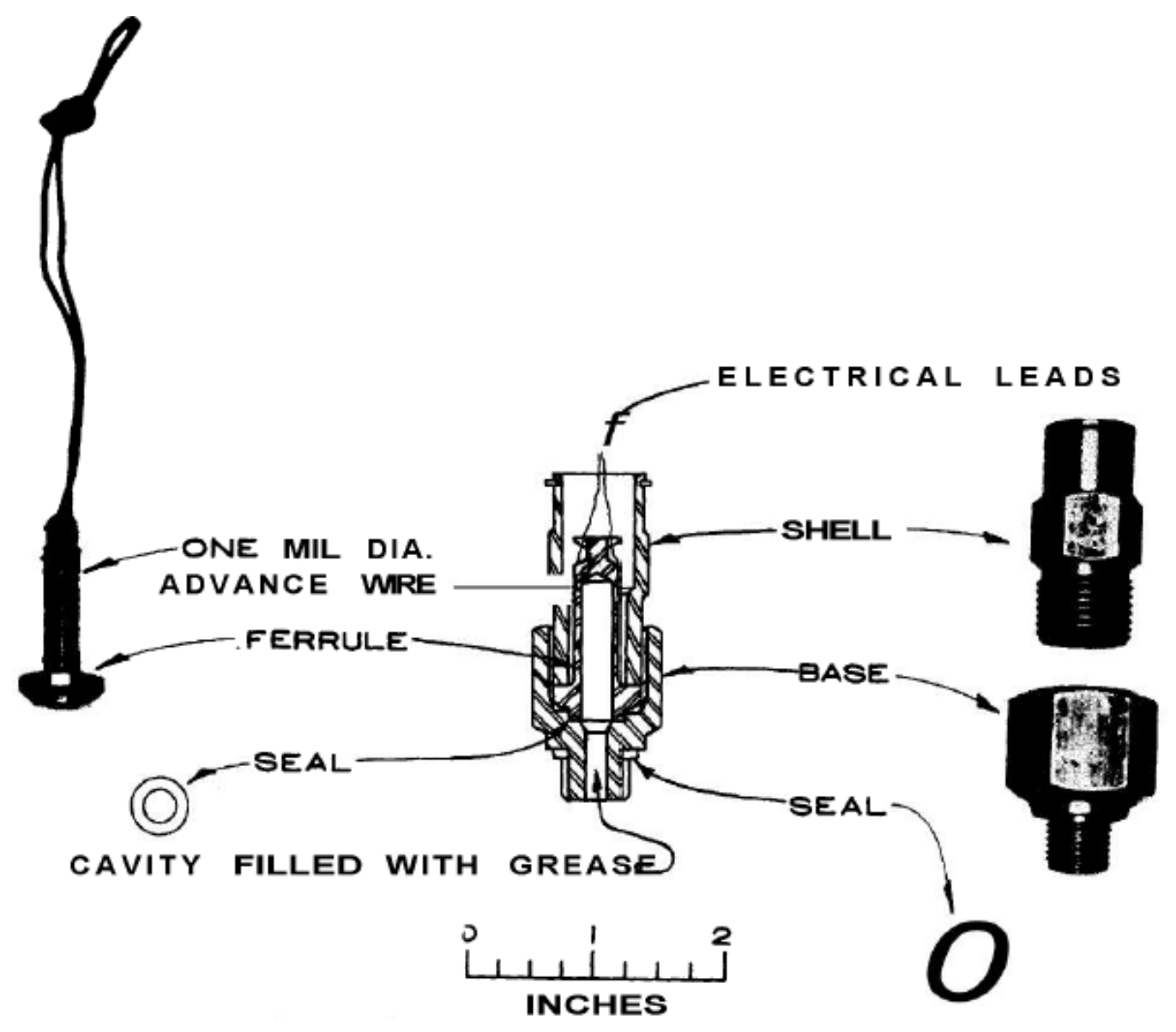

Figure 2.6: Image depicting the design of a Strain Gauge. Adapted from Jones 1965 (15).

\subsection{Measuring Projectile Velocity}

The easiest and most common way of measuring projectile velocity is by utilizing a chronograph. Chronographs allow for the measurement of projectile velocity as it passes through the screens. Optical sensors within the screens detect the projectile as it passes. The projectile will pass through two screens and the time it takes to travel between them is measured. This can be used to determine projectile velocity a short distance from the muzzle and muzzle velocity can be calculated. 


\subsection{Theoretical Evaluation of Internal Ballistics}

The firing process of a firearm can be evaluated theoretically by mathematical methods from a variety of fields such as physics and thermodynamics. Multiple methods have been developed for evaluation of the firing process and include the Vallier-Heydenreich method as well as the lumped parameters method (19). The former is based on data tables and is good for quick and approximate estimation of interior ballistics but work best in conjunction with experimental processes in which the maximum pressure and muzzle velocity are determined (19). The latter method utilizes projectile dynamics and thermodynamics of gases to compute theoretical evaluation of internal ballistics. Of these two methods, the lumped parameters method is preferred.

The lumped parameters method asserts that propellant combustion follows Roberts Law which states:

$$
\frac{d f}{d t}=\left(\frac{-B}{W e b}\right) p^{a}
$$

where $d f / d t$ is the web fraction, $B$ is the burn rate constant, $a$ is the pressure index, and $W e b$ is the web thickness (19). In this instance, the web represents the fraction of unburnt powder. In order to incorporate the geometry of the powder, following formula is used:

$$
z=(1-f)(1+k f)
$$

where $z$ represents the form function and $k$ represents the form function coefficient which describes the geometric identity of the powder (19). Utilizing these equations, the mass of the gas produced from combustion can be calculated using:

$$
m_{g}=m_{p} z
$$

where $m_{g}$ is the mass of the gas evolved and $m_{p}$ is the mass of the propellant (19). Continuing from this equation, the Nobel-Abel equation of state can be utilized from equation 2.1. The pressure can then be calculated by beginning with the first law of thermodynamics which states:

$$
Q=\Delta U+W+E_{l} \text { ost }
$$

where $Q$ is the energy produced by the combustion of the powder, $W$ is the kinetic energy of the projectile, $U$ is the internal energy of the gas, and $E_{l}$ ost is the energy lost (19). These terms can then be expressed in the following equations:

$$
\begin{gathered}
Q=\frac{m_{g} F}{\gamma-1}+E_{i} \\
W=0.5 M V^{2}
\end{gathered}
$$




$$
\begin{gathered}
\Delta U=\frac{P}{\gamma-1}\left(V o l_{g}-m_{g} c\right) \\
E_{l} \text { ost }=0.26 \frac{m_{g} F}{\gamma-1}
\end{gathered}
$$

For each of the above equations, $F$ is the impetus of the propellant, is the ratio of the heats of the gas and $E_{i}$ is the energy of the igniter (19). The volume of gas evolved, $V_{g}$, can be found using the equation:

$$
V_{g}=V_{c c}-\frac{m_{p}}{\rho}+\pi \frac{D^{2}}{4} S+\frac{m_{g}}{\rho}
$$

where $V_{c c}$ is the volume of the cartridge case, $\rho$ is the density of the propellant, $D$ is the projectile diameter, and $S$ is the projectile position (19). The pressure can then be written as:

$$
P=\frac{(\gamma-1)\left(Q-W-E_{l} \text { ost }\right)}{V_{g}-m_{g} c}
$$

By utilizing the above equations, many of the factors regarding internal ballistics can be calculated. Aside from these, the relationship between muzzle velocity and chamber pressure is of great interest and the beginnings of determining this relationship stems from the following equations:

$$
\begin{gathered}
V_{p}=2\left(\frac{2 P_{m} A L}{C_{f} m}\right)^{1 / 2} \\
P_{m}=\frac{m V_{p}^{2}}{8 A L}
\end{gathered}
$$

where $V_{p}$ is the projectile velocity, $P_{m}$ is the peak pressure, $A$ is the Cross-Sectional area of the bore, $L$ is the barrel length, $C_{f}$ is a correction factor to account for variables such as friction and rotational energy, and $\mathrm{m}$ is the bullet mass (20). These equations are basic examples of the relationship between muzzle velocity and pressure and will be expanded upon.

\subsection{IBIS $^{\circledR}$}

The Integrated Ballistic Identification System (IBIS $\left.{ }^{\circledR}\right)$ produced by Ultra Electronics Forensic Technology is a computerized imaging system used in the comparison of fired bullets and cartridge casings. This automated system takes photographs of the suspect bullets or casings and inputs them into the National Integrated Ballistic Information Network (NIBIN) program which is a database of fired cartridge casings and bullets (21). The photographs are then compared to others throughout the database. The system then 
computes a match score indicating which other bullets or casings match the questioned one and how similar the two are. This can be used to link a single firearm to multiple incidents in a short amount of time.

\subsection{Pressure Trace II $^{\mathrm{TM}}$}

To measure the change in chamber pressure during firing, the PressureTrace $\mathrm{II}^{\mathrm{TM}}$ system may be utilized. Pressure trace is a high-speed physical event data acquisition system that records the pressure change inside the chamber of a firearm in real time (22). It works by utilizing a strain gauge and PC software the simulates an oscilloscope to record data which is sent to the $\mathrm{PC}$ via a Bluetooth wireless connection. This data is the presented in the form of a pressure curve detailing the total energy of the shot and time to peak pressure. This allows for the comparison of variances between pressure curves between shots and can allow one to investigate how variables such as seating depth and neck tension affect pressure.

\subsection{Quickload ${ }^{\mathrm{TM}}$}

The Quickload ${ }^{\mathrm{TM}}$ program developed by NECO is a load development software the allows the user to input the specifications of the load they wish to develop and will return data regarding velocity, pressure, and ballistic data for that set of specifications. It contains over 1200 cartridges, 250 powders, and 2500 bullets that the user can utilize to develop their load (23). This will allow the user to gather theoretical ballistic data for a given load that can then be compared to experimental data. This will allow for comparison of ballistic variables depending upon the accuracy with which the program may estimate the ballistic parameters.

\section{$2.14 \mathrm{R}^{\circledR}$ and RStudio ${ }^{\circledR}$}

To help develop statistical models for theoretical evaluation of variables such as the chamber pressure and muzzle velocity as well as evaluate the markings left on the casings by the breech of the firearm, the $\mathrm{R}^{\circledR}$ and RStudio ${ }^{\circledR}$ programs may be used. These programs are free programs used for statistical computing and graphics $(24)$. RStudio ${ }^{\circledR}$ expands upon the utility of the $\mathrm{R}$ program by offering a console, syntax-highlighting editor with direct code execution, and tools for plotting, debugging, and workspace management (25). These program will be utilized to develop code that will allow for the theoretical calculation of chamber pressure and muzzle velocity as well as determining the relationship between the two. 


\section{Studies}

Carlucci and Jacobson discussed the theories, laws, and mathematics involved within the examination of internal ballistics. Their discussion included the use of the Lagrange Gradient and Chambrage Gradient. They produced equations for both methods and described the assumptions taken by both. The Lagrange Gradient measures the pressure at the breech of the firearm by relating it to the base pressure but neglects bore resistance and assumes that the chamber and bore diameters are the same (5). This becomes more problematic in short, large diameter chambers such as those found in handguns. This is compensated for in their discussion of the Chambrage Gradient which describes a method that accounts for the difference in barrel and chamber dimensions.

Cronemberger et al. discussed and studied multiple methods of determining the internal ballistics. They utilized the Vallier-Heydenreich method based on empirical data tables, the lumped parameters method based on a system of equations accounting for propellant combustion, thermodynamics of evolving gases, and bullet dynamics, and a commercial software known as PRODAS (Arrow Tech). They discussed and showed the equations of the first two methods which were later used to theoretically calculated the parameters found in their study. They then utilized the PRODAS software to gather experimental data. They then compared their experimental data gathered using the PRODAS software to their theoretical results using the other two methods and found that the lumped parameters method resulted in the best results for the maximum pressure and muzzle pressure (19).

Wagoner studied the effect of propellant temperature on muzzle velocity (12). It was found that the propellant temperature before firing has a significant effect on muzzle velocity. This was done by using improved military rifle (extruded single-perforated tube) and ball type propellants to determine a muzzle velocity propellant temperature coefficient via a least squares fitting technique. Although the two propellants showed variation in reaction due to the change in temperature, it was determined that they followed similar trends and a single coefficient could be used to calculate the change in muzzle velocity due to temperature (12).

Jang et al. studied the effects of propellant position inside the cartridge in relation to the pressure produced during firing (9). They observed three different instances, one in which the powder was positioned at the breech, one in which it was positioned at the base of the projectile, and one in which it was distributed evenly throughout the cartridge. They found that various phenomenon would occur depending on the position of 
the powder. When the powder was positioned near the breech, the breech pressure would ride immediately with the pressure at the base of the projectile rising shortly after. This also produced a pressure wave that propagated toward the projectile base and back toward the breech resulting in oscillations in the pressure on a graph of pressure vs. time and the pressure tends to be higher in this instance. With the powder uniformly distributed, the pressure rises uniformly and no oscillations were observed. Finally, with the powder located near the base of the projectile, the pressure at the base increases immediately and results in the projectile moving more quickly. This leads to a decrease in the mean pressure in the chamber and intensity of the pressure wave oscillations (9).

Degirmenci studied the effects of the size of powder grains and their temperature on the internal ballistics in a firearm (14). This paper discussed the factors that affect burn rate and found that temperature of the chamber, pressure, propellant composition, and grain size play a role in determining it. It was also noted that the burn rate increases as pressure and temperature increases. For the study, the propellant was separated base on diameter and the temperature of the propellant was varied from $-60 \mathrm{o} C$ to $60 \mathrm{o}$ C. It was concluded that an increase in propellant temperature and decrease in grain size leads to an increase in burn rate and pressure (14). 


\section{Methods}

For this study, two calibers were used and ammunition was reloaded to ensure known and consistent parameters of the cartridges. The calibers consisted of .38 Special and .357 Magnum. The were fired out of a T/C Contender G2 fitted with a .357 Magnum caliber barrel. This firearm was chosed due to the ability to change the barrels while using the same receiver. This allows multiple calibers to be fired while using the same breech face. The barrel was outfitted with a strain gauge to measure the chamber pressure during firing. The muzzle velocity was also recorded for each shot by utilizing a MagnetoSpeed Ballistic chronograph. After gathering the data using the Contender, an attempt to establish a relationship between muzzle velocity and chamber pressure was undertaken.

\section{1 $\mathrm{R}^{\circledR}$ Script Development}

For the purposes of modeling the internal ballistics within the firearm system, equations from the Handbook on Weaponry (26) were utilized. These consisted of the following:

$$
A=\frac{\pi}{4} D^{2}+n_{z} b_{z} t_{z}
$$

where $D$ is the caliber in meters, $n_{z}$ is the number of rifling grooves, $b_{z}$ is the width of the rifling grooves, and $t_{z}$ is the depth of the rifling grooves in meters.

$$
V_{B}^{\prime}=V_{B}-\frac{m_{c}}{\rho_{c}}
$$

where $V_{B}^{\prime}$ is the volume inside the empty casing volume, $m_{c}$ is the charge weight in kilograms, and $/ r h o_{c}$ is the density of the solid propellant.

$$
\xi_{e}=\frac{A}{V_{B}^{\prime}} x_{e}
$$

where $\xi_{e}$ is a dimensinless variable relating to projectile travel and $x_{e}$ is the projectile travel.

$$
m^{\prime}=m_{p}+\epsilon m_{c}
$$

where $m_{p}$ is the projectile mass and $\epsilon$ is a weighting factor set to 0.5 . 
The above equations are used to calculate the general quantities within the firearm system are required for calculating the internal ballistics of the shot. The following equations were used to estimate the variables up to powder burnout:

$$
\vartheta=1-\frac{1}{(1+\xi)^{\frac{k-1}{2}}}
$$

where $k$ is the ratio of specific heats of the gas.

$$
\vartheta_{b}=\frac{p_{o}^{2} A^{2}}{2 B_{a}^{2} m^{\prime} m_{c} Q_{e x}}
$$

where $B_{a}$ is the burning coefficient, $p_{o}$ is the normal pressure, $B_{a}$ is the burning coefficient, and $Q_{e x}$ is the specific heat of explosion.

$$
x^{\prime}=\frac{2 B_{a} m_{c} Q_{e x}}{p_{o} A} \vartheta
$$

where $x^{\prime}$ is the velocity of the projectile.

$$
z=\frac{B_{a} m^{\prime}}{p_{o} A} x^{\prime}
$$

where $z$ is the amount of propellant burnt.

The effective kinetic energy of the projectile and amount of energy released by the propellant can the be calculated using:

$$
\begin{aligned}
& K E=\frac{m^{\prime}}{2} x^{\prime 2} \\
& E=m_{c} Q_{e x} z
\end{aligned}
$$

The difference between these two variables divided by k-1 and the gas volume gives the pressure

$$
\begin{gathered}
G V=\frac{V_{b}^{2}+A x}{k-1} \\
P=\frac{E-K E}{G V}
\end{gathered}
$$

After burnout, the equations change as there is no longer any charge being converted in gas:

$$
\begin{gathered}
\zeta=\frac{m^{\prime}}{2 m_{c} Q_{e} x} x^{\prime 2} \\
\psi=1-\frac{1}{(1+\xi)^{k-1}}
\end{gathered}
$$




$$
\vartheta_{b}=\frac{p_{o}^{2} A^{2}}{2 B_{a}^{2} m^{\prime} m_{c} Q_{e x}}
$$

These equations are then used to calculate the gas pressure and velocity within the barrel between burnout and muzzle.

This set of equations was compared to the pressure-time curves generated by the Quickload $^{\mathrm{TM}}$ program as well as experimental data collected during firing. The $\mathrm{R}$ script used can be found in Appendix B.

\subsection{Ammunition and Reloading}

Ammunition was reloaded to similar specifications with variations in powder type and weight as well as primer type. For any given powder and primer combination, three different charge weights were used ranging from the minimum suggested charge weight from the manufacturer to the maximum charge weight suggestion. One charge weight between these two extremes was chosen as well. All charge weights were found in a Sierra Reloading Handbook and consisted of 3.4, 4.1, and 4.8 grains of Alliant Bullseye as well as 7.4, 7.8, and 8.2 grains of Alliant Blue Dot for 38 Speical. Charge weights for the .357 Magnum cartridges were 6.2, 6.7, and 7.2 grains of Alliant Bullseye and 11.6, 12.0, and 12.6 grains of Alliant Blue Dot. Powder charges were dispensed using a RCBS ChargeMaster Combo and weighed on a Denver Instrument APX-323 to ensure consistency between charges. This was repeated ten times for each primer/charge weight combination. All cartridges were reloaded on a RCBS Summit Single Stage Press. The primer types used consisted of: Sellier and Bellot, Remingtion, Tulammo, and Federal. The case over-all length (OAL) and cartridge over-all lengths (COAL) were set to SAAMI specifications and were 1.150 OAL and 1.500 COAL for 38 Speical and 1.280 OAL and 1.580 COAL for 357 Magnum. Each casing and cartridge was measured with calipers to ensure consistent OAL and COAL. Since no charge weights were duplicated between any of the casings, differentiation between caliber-powder-charge weight was made simpler. The program Quickload $^{\mathrm{TM}}$ was used to help estimate the ballistics for each load. 
Table 1: Variables for cartridges needed

\begin{tabular}{|c|c|l|}
\hline Variable & Number of Categories per Variable & Values \\
\hline Caliber & 2 & .38 Special, .357 Magnum \\
\hline Firearms per Caliber & 1 & T/C Contender \\
\hline Powder & 2 & $\begin{array}{l}\text { Alliant Bullseye, Alliant Blue } \\
\text { Dot }\end{array}$ \\
\hline Primer Type & 4 & $\begin{array}{l}\text { Sellier and Bellot, Remington, } \\
\text { TulAmmo, Federal }\end{array}$ \\
\hline Charge Weight & 3 & $\begin{array}{l}\text { Three differing charge weights } \\
\text { for each powder type per cal- } \\
\text { iber }\end{array}$ \\
\hline Shots & 10 & $\begin{array}{l}\text { Ten repetitions for each } \\
\text { charge weight }\end{array}$ \\
\hline Cartridges Needed & 480 & \multicolumn{2}{|}{} \\
\hline
\end{tabular}

\subsection{Firing and Data Collection}

During firing, chamber pressure readings inside the T/C Contender chamber were taken with a strain gauge and recorded using the PressureTrace $\mathrm{II}^{\mathrm{TM}}$ software. The muzzle velocity was recorded using a MagnetoSpeed Ballistic chronograph. The fired cartridge casings were collected and input into the IBIS system for comparison. Correlation scores between and within each charge weight/primer type combination were obtained to screen for variability based on the pressure generated during firing. During the firing process of the first round of cartridges, the MagnetoSpeed chronograph failed to save the muzzle velocities requiring a second round of cartridges to be loaded and fired. These were loaded to the same specifications as the first round. A Lee Load-Master Progressive Press was used for cartridge re-sizing, trimming, and beveling. All other loading steps were conducted in the same manner as the first round resulting in a total of 960 shots.

\subsection{IBIS $^{\circledR}$ Data Collection}

After firing, each casing was entered into the the IBIS ${ }^{\circledR}$ system and correlations between the casings were run. Since the IBIS ${ }^{\circledR}$ system runs correlations in a pairwise fashion and does not compare casings already in the database to those entered after them, the correlations were deleted and re-run to ensure all casings were compared to one another. After completion of the re-correlations, the data was extracted into a Microsoft ${ }^{\circledR}$ Excel ${ }^{\circledR}$ file. The files were then run through an $\mathrm{R}$ script to remove any duplicate comparisons that may have been present. A second $\mathrm{R}$ script was then run to combine all individual data files into one. 


\subsection{Pressure Trace $\mathrm{II}^{\mathrm{TM}}$}

The data collected by the Pressure Trace $\mathrm{II}^{\mathrm{TM}}$ system consisted of plots representing the pressure as a function of time during firing. These were converted to a Microsoft ${ }^{\circledR}$ Excel ${ }^{\circledR}$ file and an $\mathrm{R}$ script was created to combine the data for each ten shot string into separate files for each shot. Due to variances in the time it took for the pressure to rise, some traces had to be adjusted so all curves aligned with one another.

Figure 4.1 shows an example of the pressure traces collected during the firing process. It can be seen that some traces begin to increase in pressure sooner than others. It was found that this is due to ignition delay of the powder and is not uncommon. For this reason, an $\mathrm{R}$ script was used to adjust all traces so they aligned with one another allowing for easier comparison.

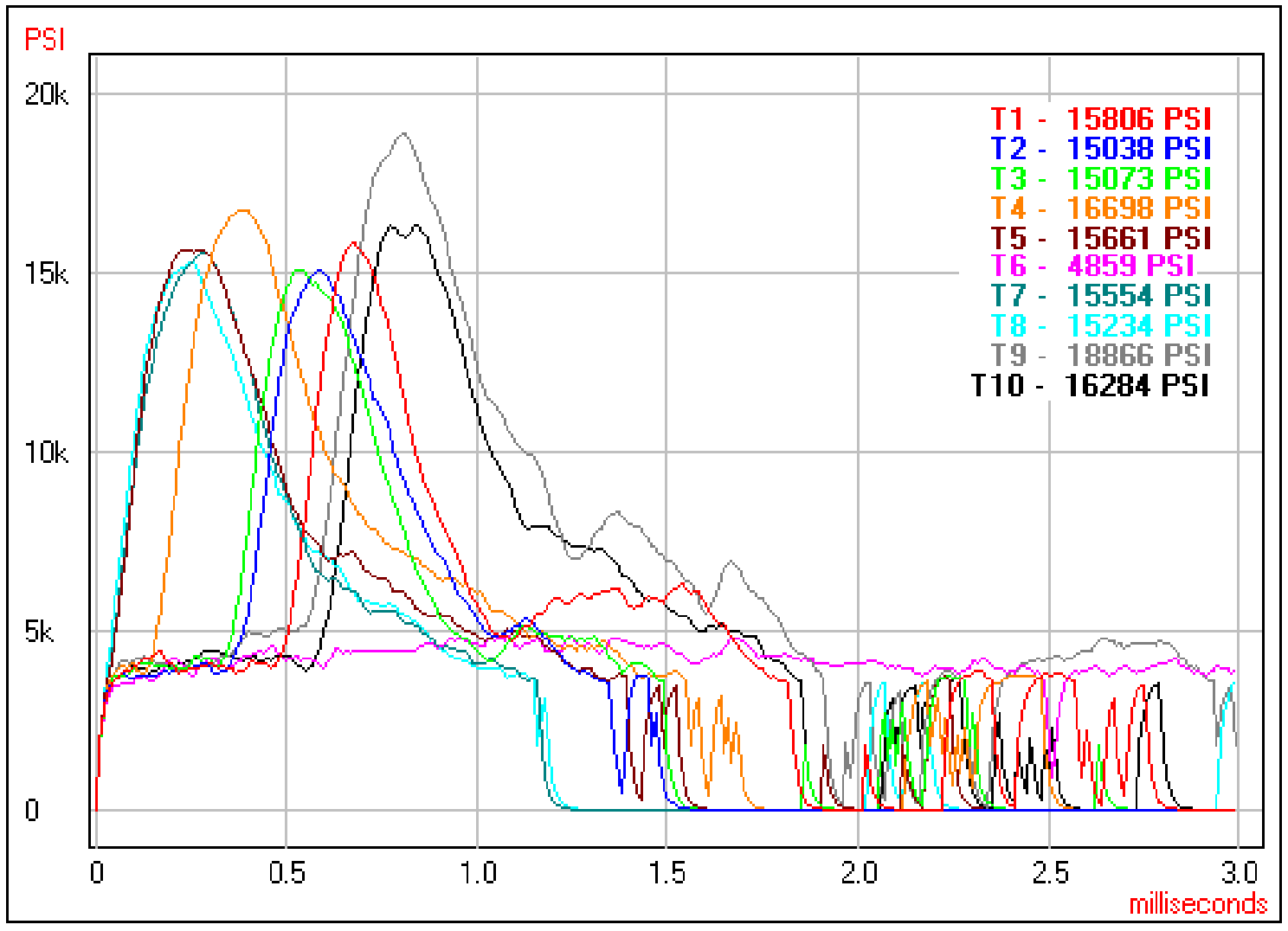

Figure 4.1: Image showing a ten shot string captured by Pressure Trace II $^{\mathrm{TM}}$ during data collection. This trace was captured using a .357 Magnum load comprised of 6.2 grains of Alliant Bullseye powder. 
Figure 4.2 shows the pressure trace data after adjustment for ignition delay. The maximum pressure for each shot was then taken from this data and added to the IBIS excel file.

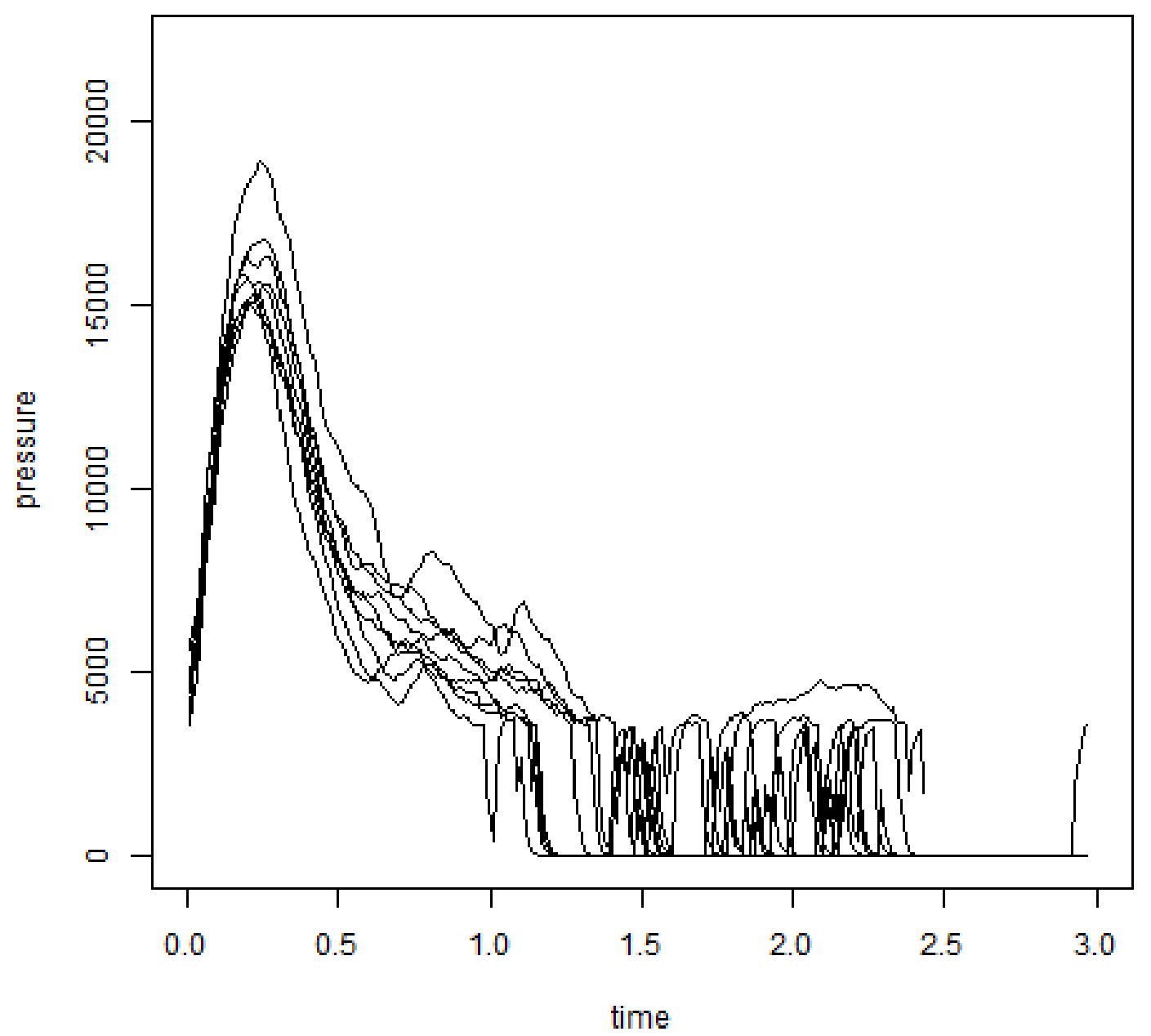

Figure 4.2: Image showing a ten shot string captured by Pressure Trace II $^{\mathrm{TM}}$ during data collection after adjustment. This trace consists of the same shots as those found in 4.1. 


\subsection{MagnetoSpeed ${ }^{\mathrm{TM}}$ Data Collection}

During firing, the MagnetoSpeed ${ }^{\mathrm{TM}}$ automatically collects muzzle velocity data as the projectile exits the muzzle. The data is saved to a microSD card and is stored in a Microsoft ${ }^{\circledR}$ Excel ${ }^{\circledR}$ file format. Muzzle velocity data for each shot was collected using this method.

\subsection{Data Analysis}

After the collection of all data, it was compiled into Microsoft ${ }^{\circledR}$ Excel ${ }^{\circledR}$ files. The maximum chamber pressure measured by the Pressure Trace $\mathrm{II}^{\mathrm{TM}}$ program was added to the IBIS file and the difference in pressure for each shot-database combination was calculated as well as average pressure for each charge weight. The same was done with muzzle velocity data collected from the MagnetoSpeed ${ }^{\mathrm{TM}}$. A R script was then created to plot the various shot parameters against each other as well as the breech face and firing pin scores returned by IBIS. The data was subset by caliber, .38 Special and .357 Magnum, and the breech face and firing pin scores were plotted against the database charge weight. These were further divided based on the sample powder type and charge weight. This allowed for the observation of breech face and firing pin scores for each powder type and charge weight compared to all other powder types and charge weights. The breech face and firing pin scores were also plotted against the delta pressure between the sample and database exhibits. Finally, the breech face and firing pin scores were plotted against each other and observed based on powder charge weight combination. All $\mathrm{R}$ scripts used can be found in Appendix B. 


\section{Results and Discussion}

During the input of casings into the IBIS ${ }^{\circledR}$ system, photographs of the breech face and firing pin impressions were taken. Visual examination of these photographs showed differences in the impressions generated by low-pressure and high-pressure casings. It was noticed that low-pressure casings showed less impression development and the impressions that were developed appeared shallower than those developed from high-pressure cartridges. This can be observed in Figures 5.1 and 5.2. 


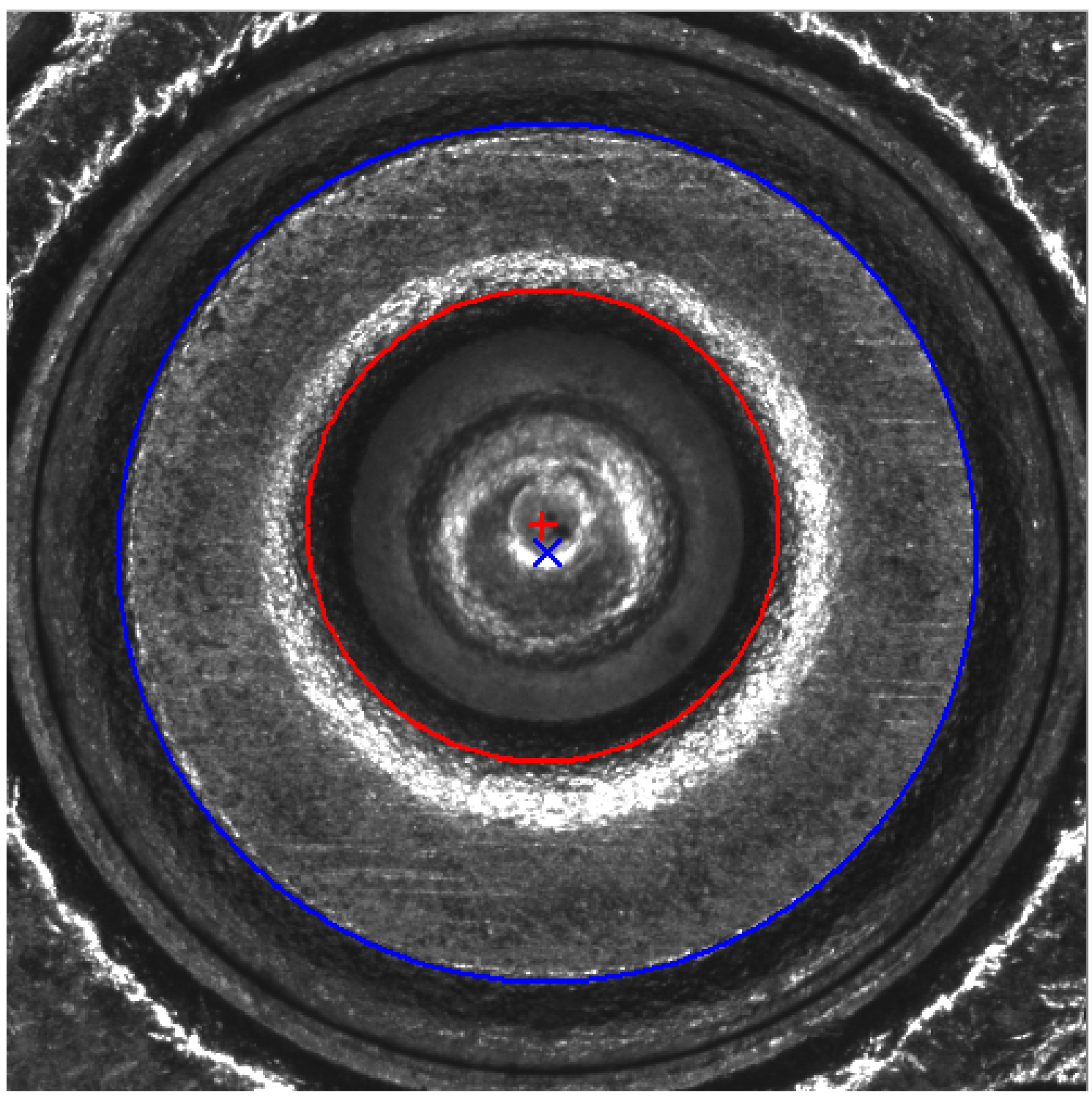

Figure 5.1: IBIS ${ }^{\circledR}$ photograph of .38 Special casing fired using 3.4 grains of Bullseye powder. 


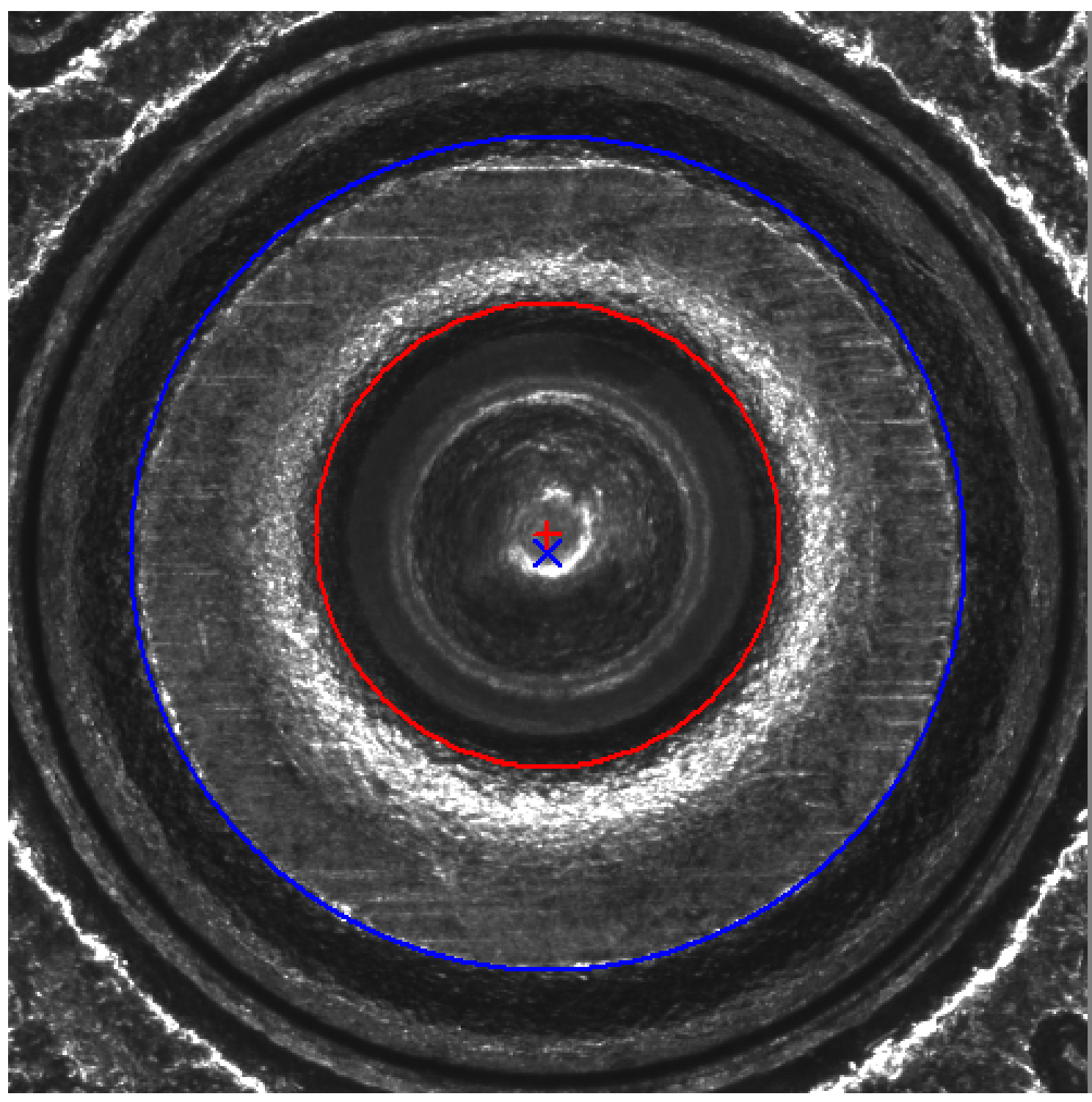

Figure 5.2: IBIS $^{\circledR}$ photograph of .38 Special casing fired using 4.8 grains of Bullseye powder.

To evaluate how accurately the Quickload ${ }^{\mathrm{TM}}$ program and $\mathrm{R}$ script estimated the internal ballistics of the system, the graphs generated by both methods were plotted with the adjusted pressure traces collected by the Pressure Trace $\mathrm{II}^{\mathrm{TM}}$ system during firing.

Figure 5.3 shows this trace for a .38 Special cartridge loaded with 3.2 grains of Bullseye 
powder. It can be seen that the Quickload ${ }^{\mathrm{TM}}$ and $\mathrm{R}$ script traces estimate the pressure to be higher than that of the experimental traces.

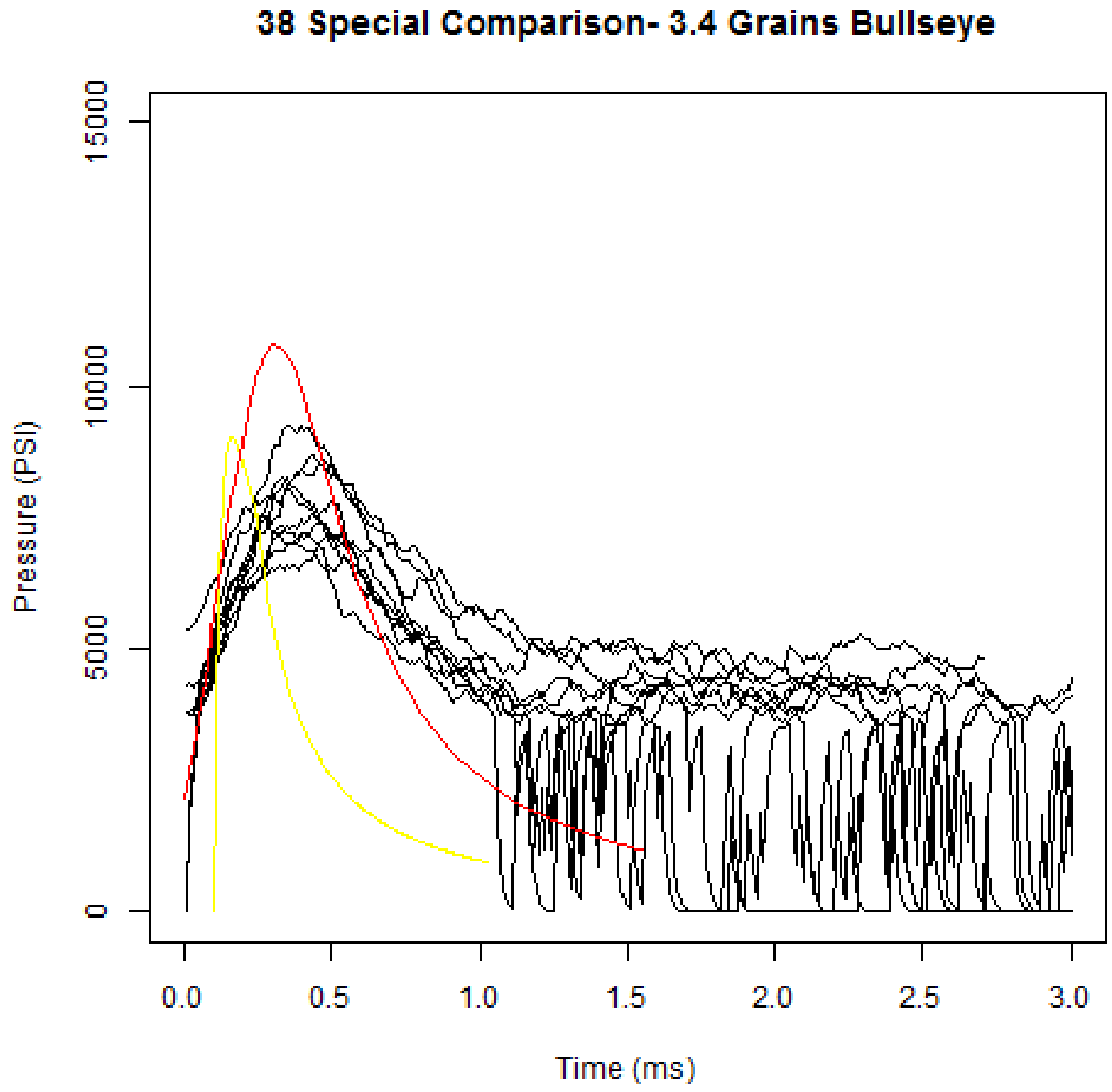

Figure 5.3: Pressure Trace graph showing the comparison of Quickload(red) trace, $\mathrm{R}$ script (yellow) trace, and traces obtained from firing (black)

This trend is observed for all powder-charge weight combinations. This may be attributed to the Quickload ${ }^{\mathrm{TM}}$ using estimation parameters based on the copper crusher 
method of pressure measurement when the experimental data was collected using a strain gage. The other figures can be viewed in Appendix A. The extreme rise in pressure seen in the $\mathrm{R}$ script estimation is believed to be attributed to the equations used not taking into account the progressive/regressive buring of the propellant. As the powder's surface area begins to diminish, the burn rate should slow which would account for the difference between the $\mathrm{R}$ script estimation, the Quickload $^{\mathrm{TM}}$ estimation, and the collected data. It should be noted that the loads for these cartridges were taken from a published reloading handbook and maximum pressure from SAAMI for .357 Magnum is 35,000 PSI. The Quickload $^{\mathrm{TM}}$ program estimates the powder and charge combinations used in this study to be well above this threshold indicating that there is a discrepancy in measurement method.

By observing Figures 5.4, 5.5, 5.6, 5.7 which show breech face and firing pin scores based on charge weight and comparing them to the average pressure per powder charge in Figure 5.1, it can be seen that as the average pressure between the charge weights increases, the breech face scores decrease.

By observing Figure 5.4, it can be seen that higher median breech face scores were observed for casings loaded with charge weights similar to those being observed. An example of this can be seen by observing data corresponding to the $3.4,4.1$, and 4.8 grain charge weights. These charge weights gave higher median breech face scores for both the Bullseye and Blue Dot loads when compared to the 11.6, 12, and 12.6 grain charge weights. This indicates that the .38 special cartridges had a higher breech face score when compared with other .38 special cartridges and stay similar as the charge weight is varied. When compared to the .357 Magnum cartridges, it can be observed that the median breech face scores are lower and decrease as the charge weight increases. This can be seen by observing the 11.6, 12, and 12.6 grain charge weights. These charges gave lower median breech faces scores when compared to the previously mentioned charge weights. It is also noted that, as the charge weight within this group increases, the median breech face score decreases. When observed in conjunction with Table 5.1, this shows that, cartridges with similar maximum pressures give higher median breech face scores than cartridges where the maximum pressure varies. Using the previous examples, the 3.4, 4.1, and 4.8 charge weights have a similar average maximum pressure when compared to one another with a difference of 3,530.6 pounds per square inch between the highest and lowest loads in this group. When compared to the 11.6, 12, and 12.6 charge weight group, the average maximum pressure varies by at least 10,068.2 psi when comparing the 11.6 charge weight to the 4.8 grain charge weight. However, this trend is not observed for the 357 Magnum data. By observing Figure 5.4 it is noticed that the median breech face scores stay relatively consistent between caliber and charge weight, the exception to this being the .357 Magnum cartridges which gave a lower average breech face score. 


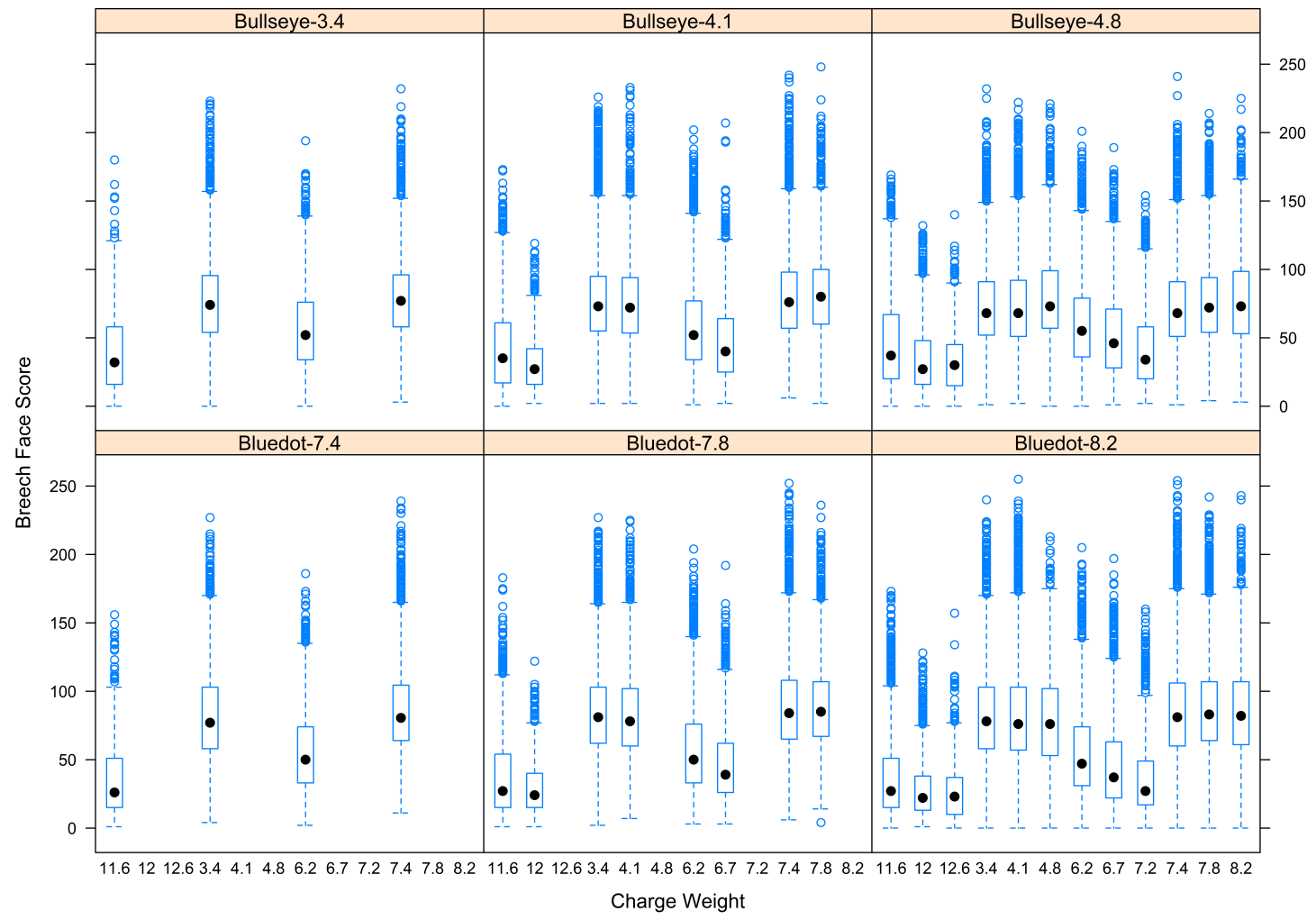

Figure 5.4: Plots showing the comparison of charge weight to breech face score for a given powder-charge combination for 38 Special. 


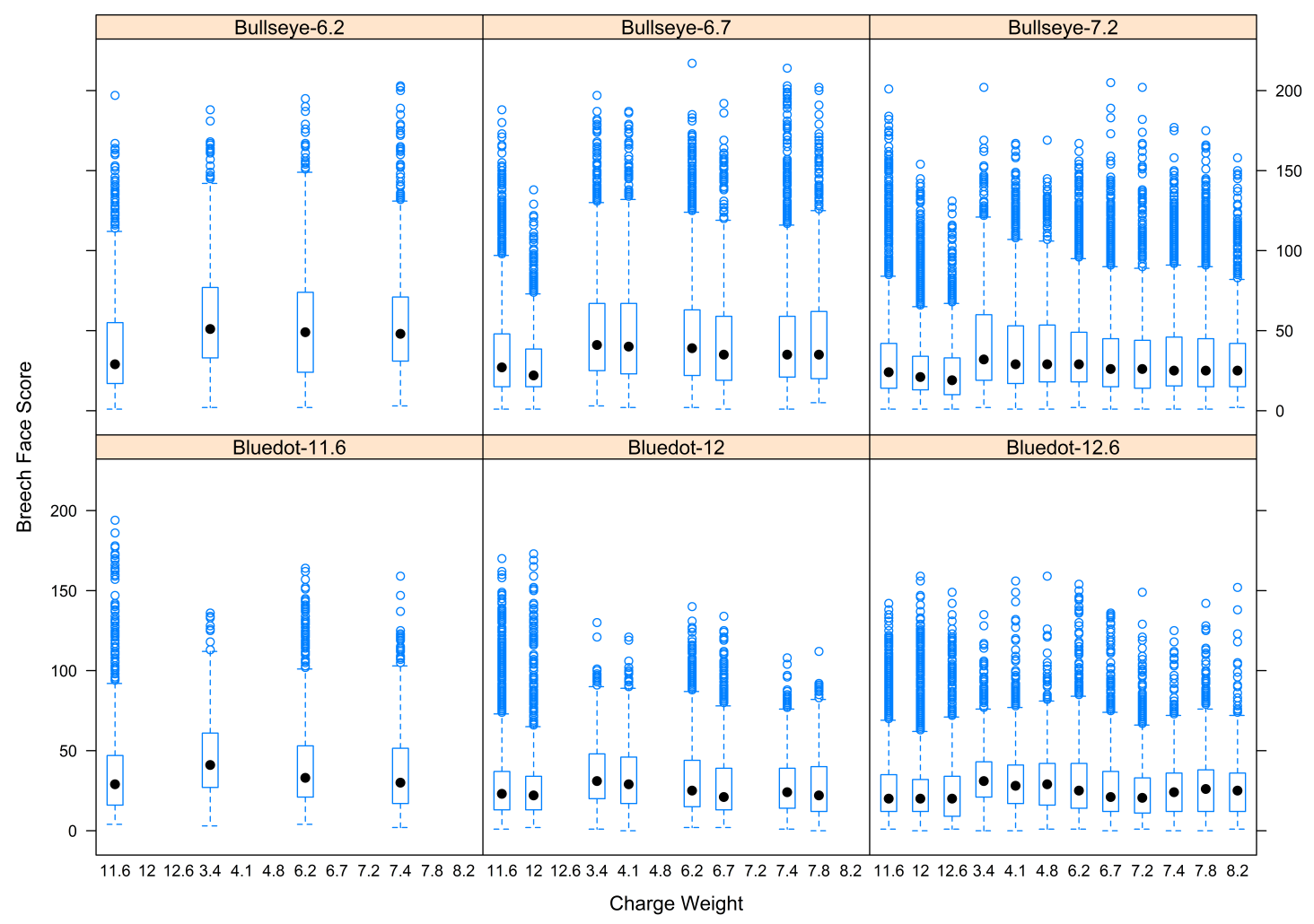

Figure 5.5: Plots showing the comparison of charge weight to breech face score for a given powder-charge combination for 357 Magnum.

By observing Figures 5.6 and 5.7, it can be seen that the median firing pin scores increased as the charge weight increases within a powder type. The median firing pin scores obtained from the .38 Special cartridges loaded with Bullseye powder increased as the charge weight increased but appear to remain relatively constant across the other powder type and charge weights. The .38 Special cartridges loaded with Blue Dot powder shows the same trend but it appears to be more pronounced than with the Bullseye poweder. However, this trend is most pronounced in the .357 Magnum data loaded with Bullseye powder which shows increasing median firing pin scores when compared to the .38 Special cartridges but relatively constant scores when compared to other .357 Magnum cartridges. When observing these trends in conjunction with table 5.1, it can be seen that the higher pressure cartridges give lower median firing pins scores when compared against lower pressure cartridges but, as the average maximum pressure difference between charge weights decreased, the firing pin scores increased. 


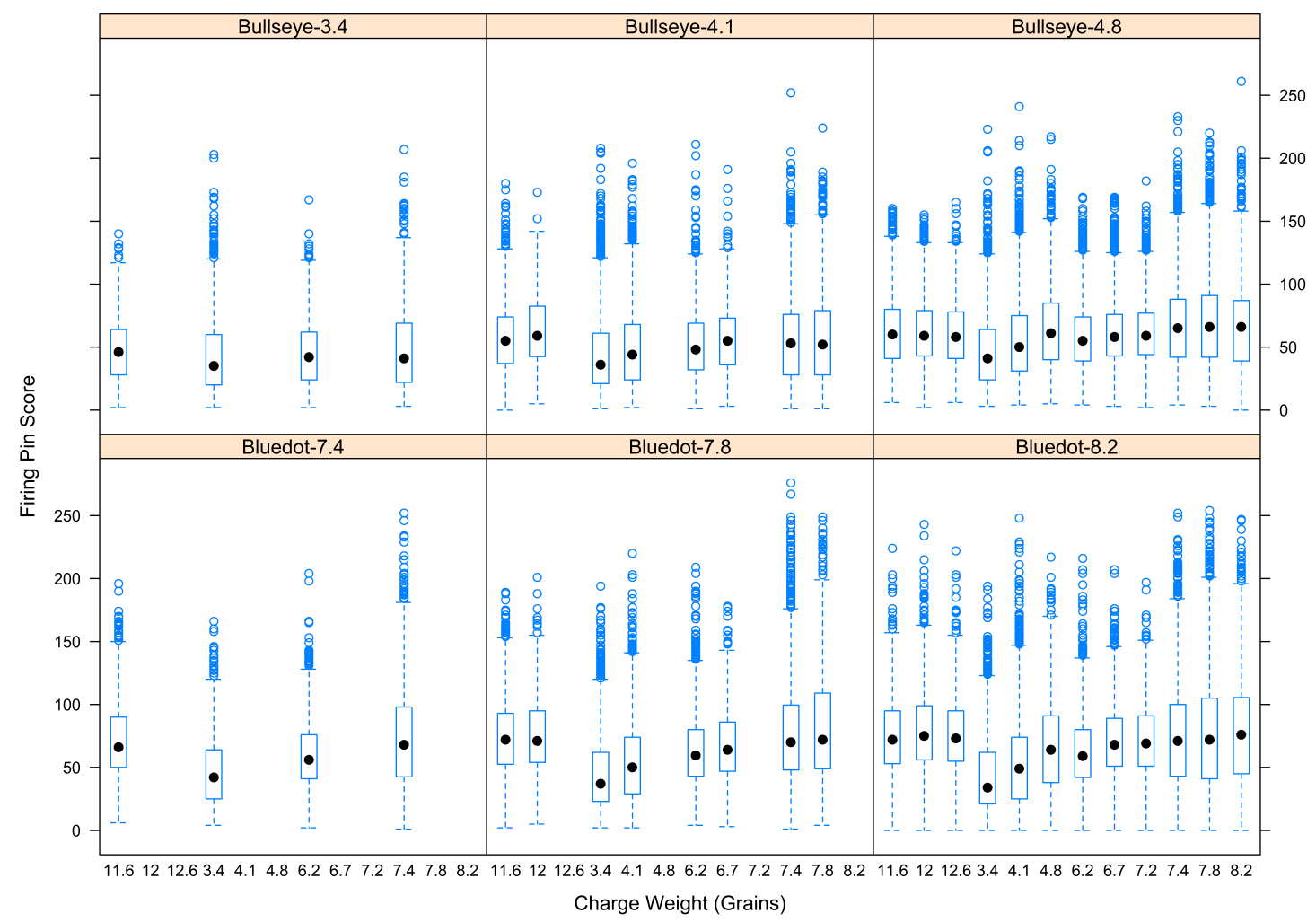

Figure 5.6: Plots showing the comparison of charge weight to firing pin score for a given powder-charge combination for 38 Special. 


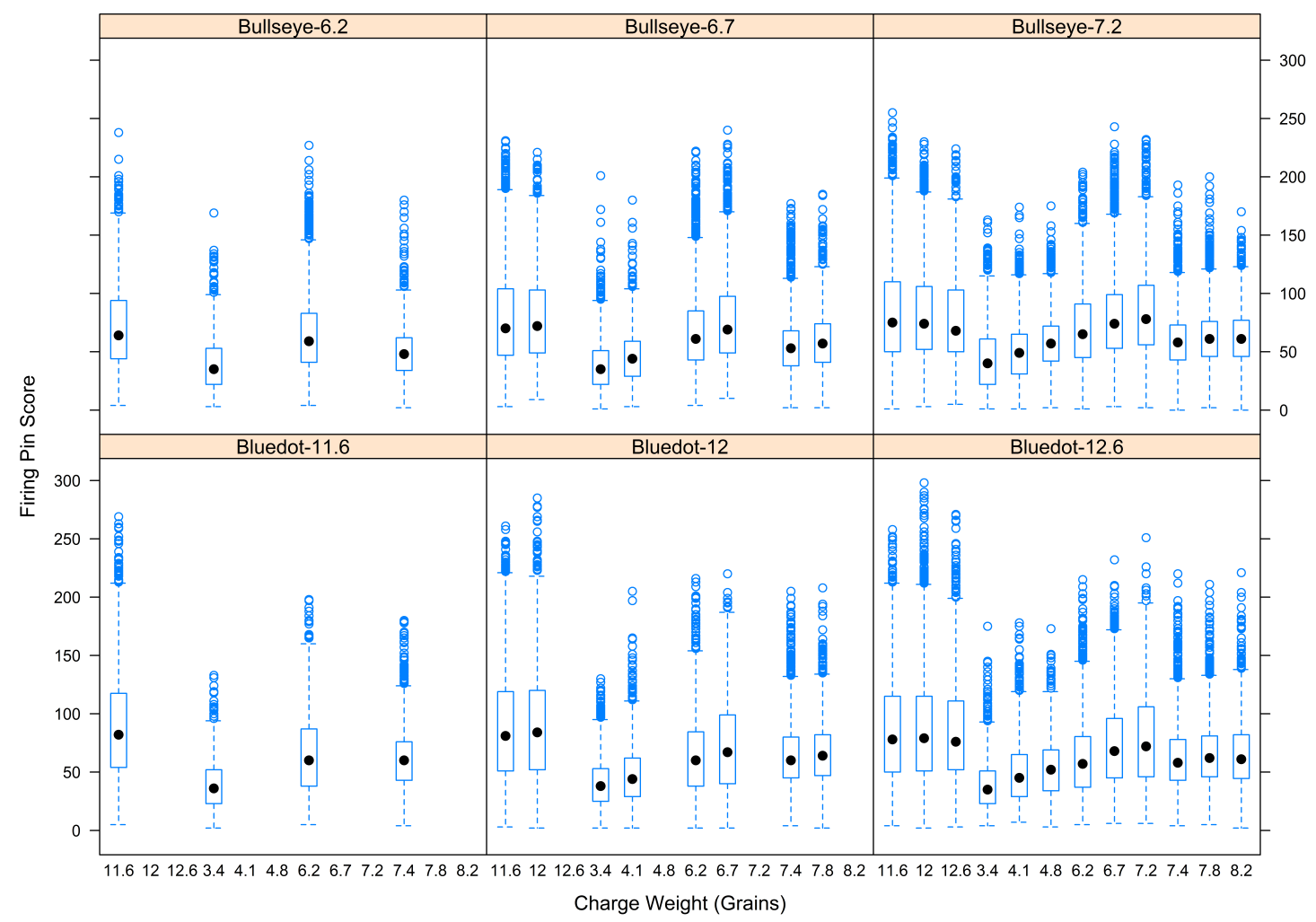

Figure 5.7: Plots showing the comparison of charge weight to firing pin score for a given powder-charge combination for 357 Magnum. 
Table 5.1: Table 2: Average maximum pressure per powder type and charge weight.

\begin{tabular}{|c|c|c|c|}
\hline Caliber & Powder Type & Charge Weight & Average Maximum Pressure (PSI) \\
\hline \multirow{3}{*}{ 357 Magnum } & \multirow{2}{*}{ Bullseye } & 6.2 & $15,634.8$ \\
& & 6.7 & $17,633.3$ \\
& & 7.2 & $19,703.2$ \\
\hline \multirow{3}{*}{ 357 Magnum } & \multirow{2}{*}{ Blue Dot } & 11.6 & $21,231.5$ \\
& & 12.6 & $22,979.4$ \\
\hline \multirow{3}{*}{ 38 Special } & \multirow{2}{*}{ Bullseye } & 3.4 & $25,273.7$ \\
& & 4.1 & $7,632.7$ \\
& & 4.8 & $8,884.9$ \\
38 Special & \multirow{2}{*}{ Blue Dot } & 7.4 & $8,851.8$ \\
& & 8.2 & $9,731.1$ \\
& & & $10,531.8$ \\
\hline
\end{tabular}

By observing Figures 5.8, 5.9, 5.10, and 5.11, it can be seen how differences in pressure between cartridges may effect the breech face and firing pin match scores. Figure 5.8 shows three groups that appear to form around different ranges of delta pressures. The first group can be found between zero and approximately 5,000 psi, the second between 5,000 and 15,000 psi, and the third from 15,000 psi upward. Within each grouping, it can be seen that the match scores increase as the variance in pressure within each group decreases. 


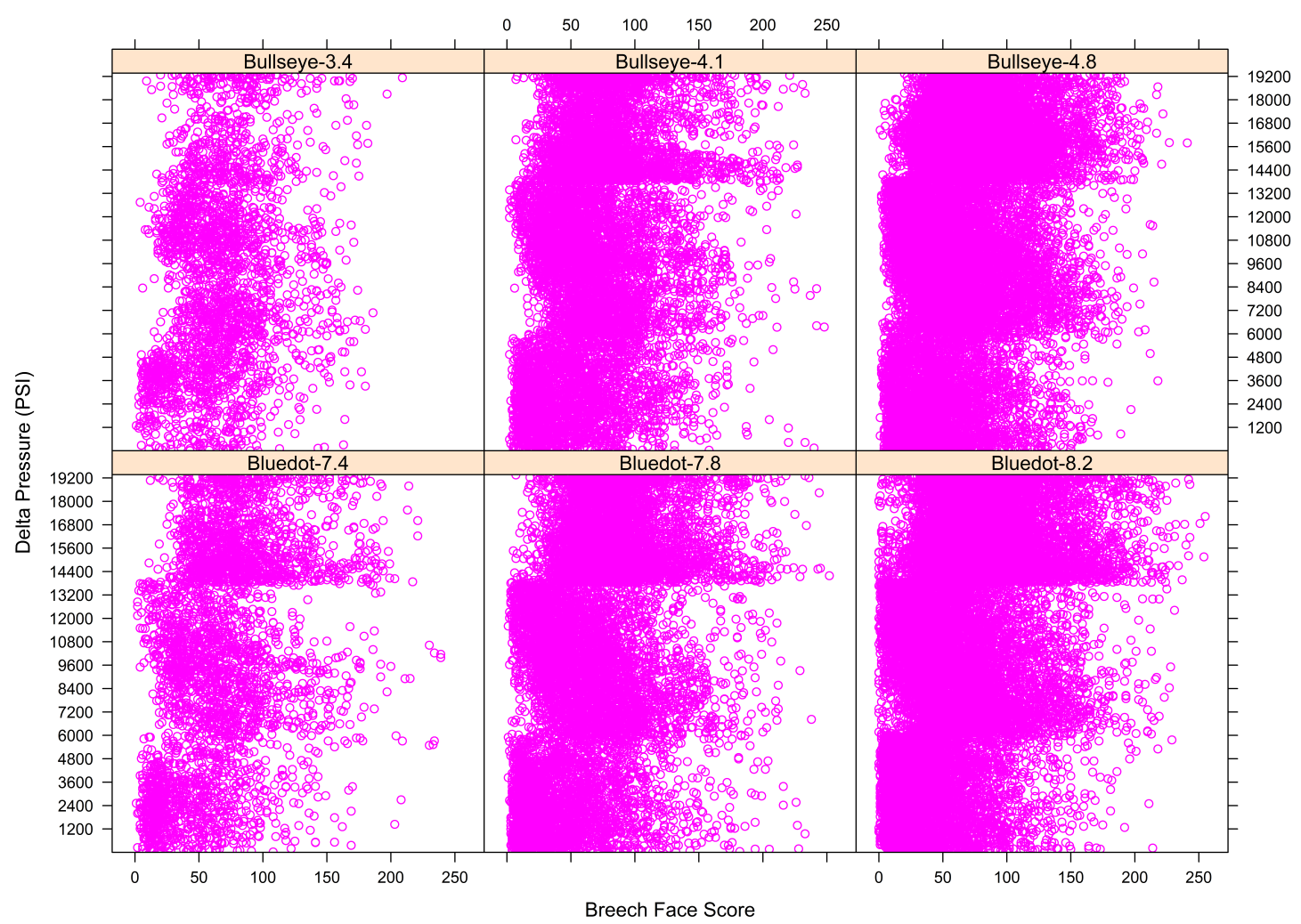

Figure 5.8: Plots showing the comparison of delta pressure to breech face score for a given powder-charge combination for 38 Special.

This trend can also be seen in Figure 5.9 for 357 Magnum. 


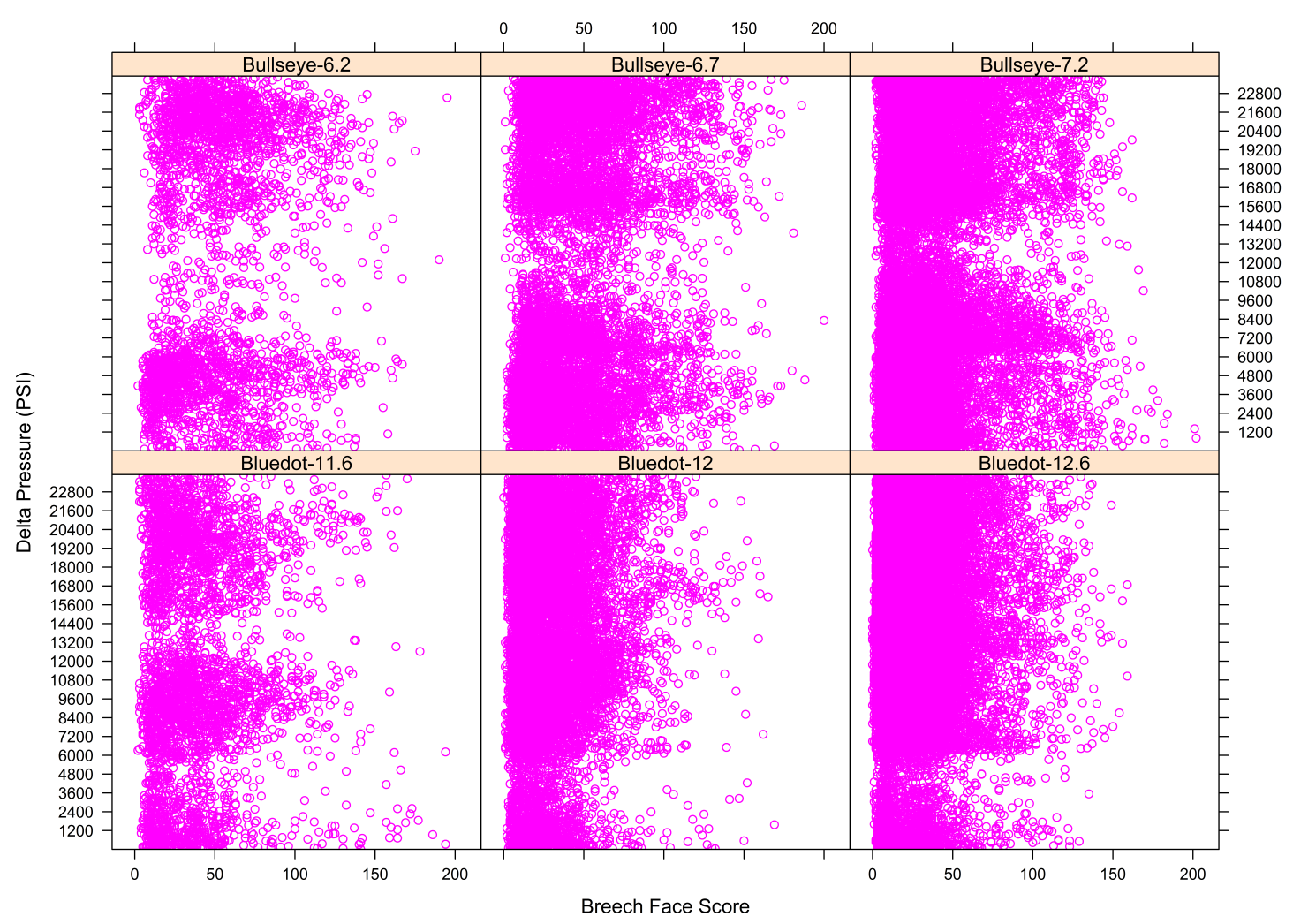

Figure 5.9: Plots showing the comparison of delta pressure to breech face score for a given powder-charge combination for 357 Magnum.

Figures 5.10 and 5.11 show how the variances in pressure between casings can effect firing pin scores. These show that the firing pin scores have less variation due to differences in pressure. However, Figure 5.10 and the bottom row of Figure 5.11 show a slight increase in firing pin scores when the difference in pressure between casings is around 15,000 psi. The top row of Figure 5.11 also shows a larger abundance of higher firing pin scores as the pressure difference decrease, but this trend does not seem consistent across all data. It is believed that these groupings appear due to the ranges within which the pressure differences may be. Since only select groups of charge weights were selected, similar groupings should result as a difference of their pressures as seen in the previously mentioned figures. 


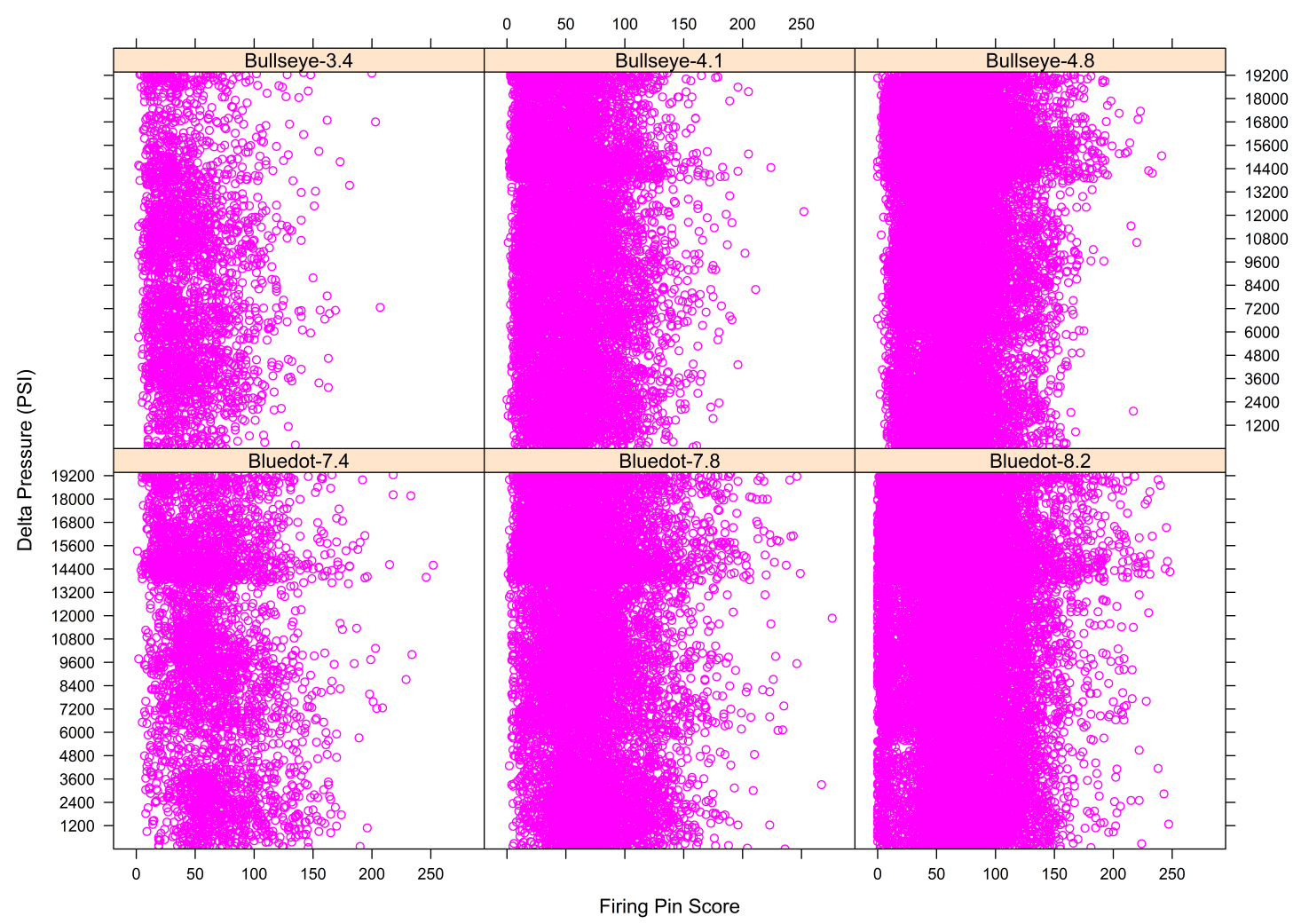

Figure 5.10: Plots showing the comparison of delta pressure to firing pin score for a given powder-charge combination for 38 Special. 


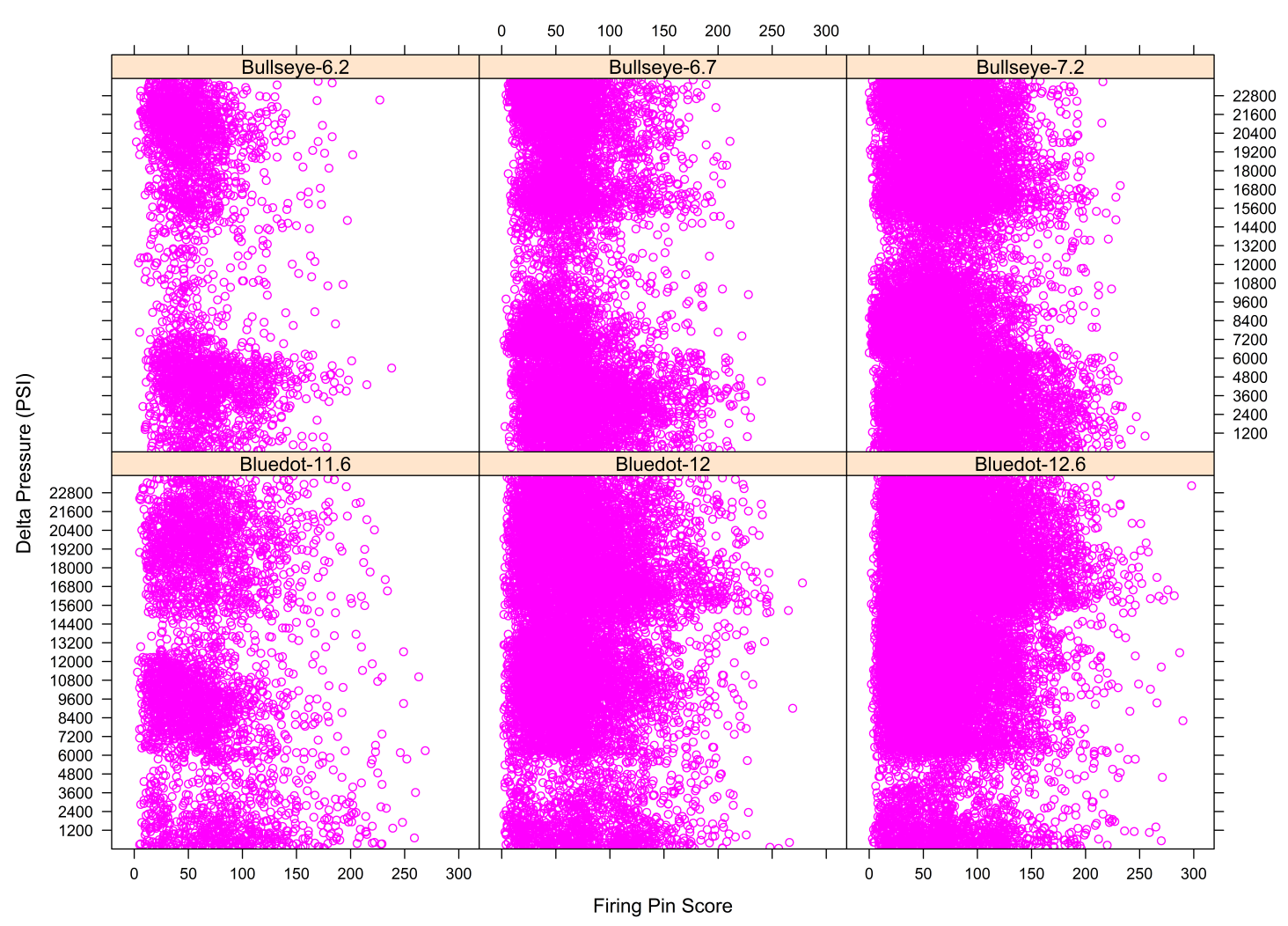

Figure 5.11: Plots showing the comparison of delta pressure to firing pin score for a given powder-charge combination for 357 Magnum.

By observing Figures 5.12 and 5.13, the effect that the difference in charge weights, and ultimately pressure, plays on the results returned by IBIS ${ }^{\circledR}$. Figure 5.12 shows the breech face and firing pin scores for a given charge load based on the results returned for .38 Special. It can be seen that, as the difference in the charge weight increases, the breech face scores decrease. By observing the plots in which the charge weight is compared to itself or one close to it such as 7.4-7.4, 8.2-7.8, or 4.1-3.4, the breech face and, in some cases, firing pin scores are greater in comparison to the scores returned when compared to a charge combination with a great difference between them. When the charge weight combination has a greater difference between them, such as 7.4-11.6, the breech face scores are lower. The firing pin scores appear to have much less variation within them compared to breech face scores. The lowest firing pin scores are observed with comparing low charge weight with high charge weights such as 3.4-11.6 or 4.1-12. However, other charge weight combinations show much less variation within the firing pin scores as compared to the 
variation in breech face scores. These trends can also be observed for .357 Magnum in Figure 5.13.

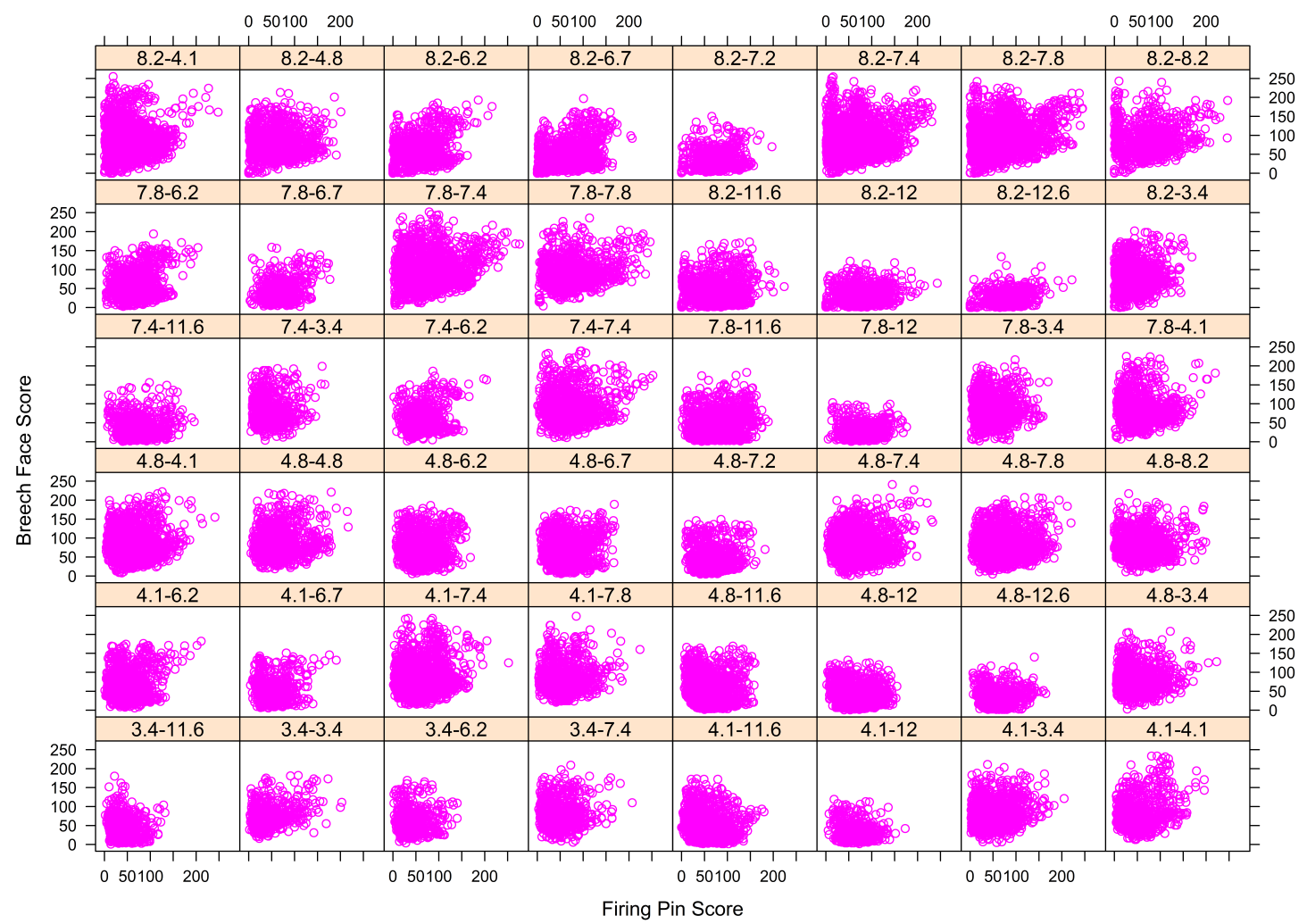

Figure 5.12: Plots showing the comparison of breech face to firing pin score for charge weight combinations for 38 Special. 


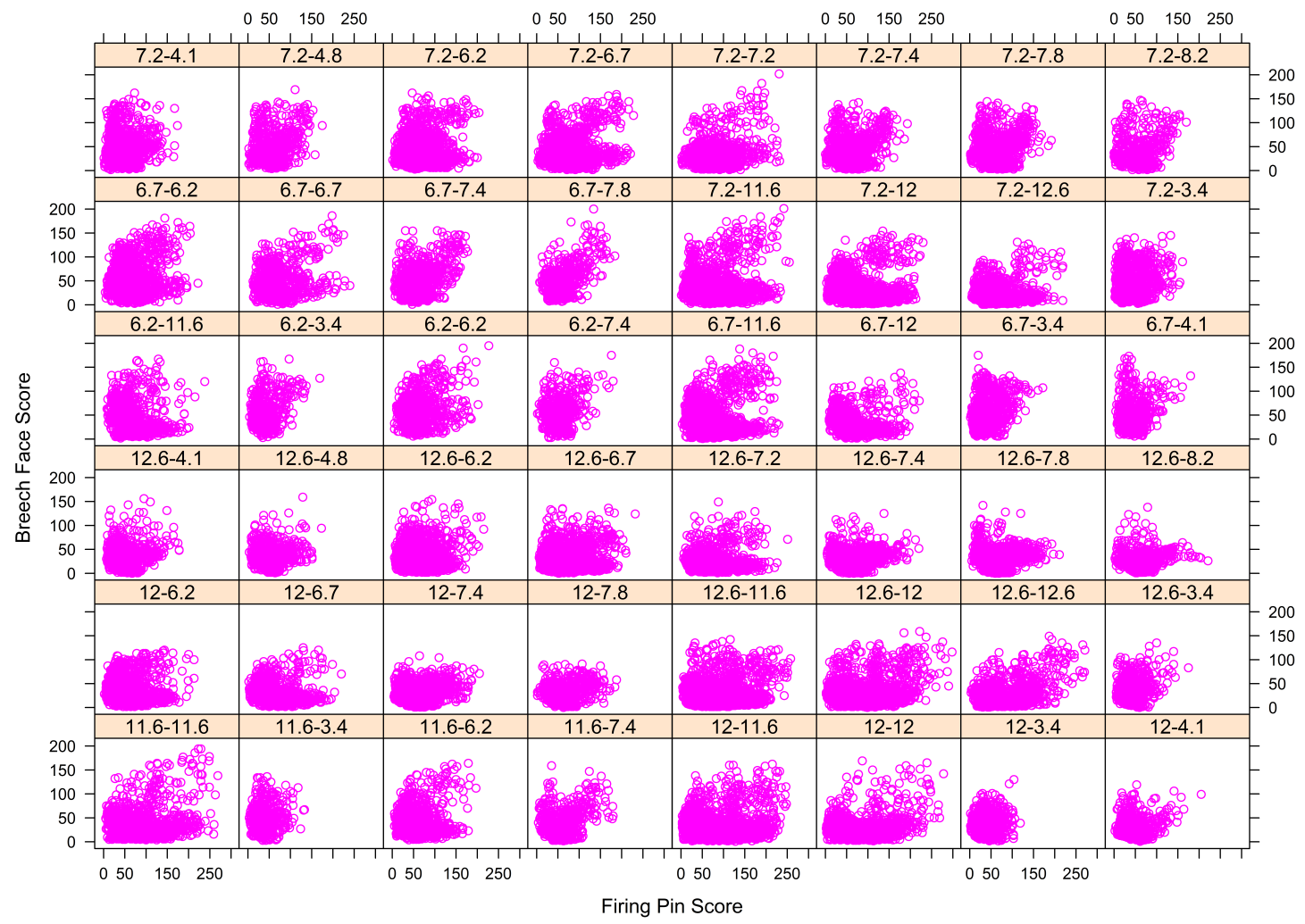

Figure 5.13: Plots showing the comparison of breech face to firing pin score for charge weight combinations for 357 Magnum. 


\section{Conclusions}

Based on the data collected during this study, it can be determined that differences in pressure between two fired cartridge casings can result in lower average match scores from IBIS $^{\circledR}$, particularly when observing breech face scores. It was observed that cartridges which had similar charge weights resulted in higher average breech face scores while cartridges with greater differences between their charge weights resulted in lower average scores. Comparing the charge weights with the pressure generated from them during firing, the greater the difference in the pressure between cartridges, the lower the scores. It was also observed that plotting delta pressure against breech face impressions gave groupings of data within which, as the difference in pressure decreased, the breech face scores appeared to increase. However, firing pin scores appeared to remain relatively constant across all comparisons with exception to the most extreme differences in pressure indicating that firing pin impressions are less susceptible to variation based on pressure than breech face impressions.

The results of this study show the importance of using similar brands and types of ammunition when conducting test fires for comparison to casings collected at a crime scene. Should an examiner use cartridges which develop pressures which vary greatly from those used in a crime, it is possible for IBIS ${ }^{\circledR}$ to return lower breech face and firing pin scores. This may result in possible identifications being overlooked or disregarded during casework. The use of similar pressure cartridges will give the examiner the most accurate results and should be utilized whenever possible. In the case of reloaded ammunition, it would be best for the examiner to load multiple cartridges with varying charge weights so they encompass more pressure ranges when comparing to crime scene casings rather than relying on a single cartridge with may not give the most accurate results.

Future research directions include the continued investigation into a theoretical method for evaluating the interior ballistics of a firearm system. Continued research should also be focused on evaluating the importance of pressure within a caliber that may have more variability to the pressure generated during firing. Investigation into whether a firearm with interchangeable barrels, such as the T/C Contender G2 pistol used in this study, will result in higher or lower breech face and firing pin scores if various calibers which generate greater differences in pressure are used and compared against one another. Finally, since there were visual differences in the cartridges which generated different pressures, it may be beneficial to investigate the possibility of determining the pressure based on the visual examination of the fired cartridge. 


\section{Bibliography}

[1] Cork DL, ebrary I, National Research Council (U S ) Committee to Assess the Feasibility A, of a National Ballistics Database TC. Ballistic imaging. Washington, DC: National Academies Press; 2008.

[2] Warlow TA, ebrary I. Firearms, the law and forensic ballistics. Bristol, PA;London;: Taylor \& Francis; 1996;2002;.

[3] Bunch SG, Smith ED, Giroux BN, Murphy DP. Is a match really a match? A primer on the procedures and validity of firearm and toolmark identification. Forensic Science Communication. 2009;

[4] Tulleners FA. Technical evaluation: feasibility of a ballistics imaging database for all new handgun sales. CALDOJ Publication. 2001;

[5] Carlucci DE, Jacobson SS. Ballistics: theory and design of guns and ammunition. Boca Raton: CRC Press/Taylor \& Francis Group; 2008.

[6] Heard BJ. Handbook of firearms and ballistics: examining and interpreting forensic evidence. vol. 1. John Wiley \& Sons; 2011.

[7] Litz B. Effects of cartridge over all legth (COAL) and cartridge base to Ogive (CBTO). Berger. 2013;.

[8] Internal Ballistics. Hornady Manufacturing. 2015;.

[9] Jang JS, Sung HG, Roh TS, Choi DW. Numerical study on properties of interior ballistics according to solid propellant positions in chamber. Proceedings of the 26th International Symposium on Ballistics. 2011;.

[10] House JE. The Gun Digest Book of .22 Rimfire: Rifles·Pistols·Ammunition. F+W Media; 2005. Available from: https://books.google.com/books?id=8Stm1d8_P6QC.

[11] Rinker RA. Understanding Firearm Ballistics: Basic to Advanced Ballistics, Simplified, Illustrated and Explained. Mulberry House Publishing Company; 2005. Available from: https://books.google.com/books?id=bgfoAAAACAAJ.

[12] Wagoner BA, MD ABRLAPG. Change in Muzzle Velocity Due to a Change in Propellant Temperature for Small Arms Ammunition. Defense Technical Information Center; 1990. Available from: https://books.google.com/books?id=lBasNwAACAAJ. 
[13] Cengel YA. Thermodynamics: An Engineering Approach. McGraw-Hill Science/Engineering/Math; 2004.

[14] Degirmenci E. Effects of grain size and temperature of dole base solid propellants on internal ballistics performance. Fuel. 2015;.

[15] Jones RN, Hitchcock HP, Villegas DR. Interior Ballistics of Guns. United States Army Material Command; 1965.

[16] Associates NAS. Propellant Properties;

[17] Longridge JA. Internal Ballistics. E. \& F.N. Spon; 1889. Available from: https://books.google.com/books?id=c9U3AAAAMAAJ.

[18] Hatcher JS. Hatcher's Notebook. Stackpole Books; 1962. Available from: https://books.google.com/books?id=yESNUKSg5aMC.

[19] Cronemberger PO, Lima Junior EP, Gois JAM, Caldeira AB. Theoretical and Experimental Study of the Interior Ballistics of a Rifle 7.62. Thermal Engineering. $2014 ;$.

[20] Calculating Barrel Pressure and Projectile Velocity in Gun Systems. Close Focus Research. 2012;.

[21] of the Inspector General O. The Bureau of Alcohol, Tobacco, Firearms, and Explosives National Integrated Ballistic Information Network Program. Audit Report. 2005;

[22] Recreational Software I. Rifle Chamber Pressure Testing Hardware \& Software. 2015;

[23] Neco. QuickLOAD Version 3.9. 2015;.

[24] Foundation TR. The R Project for Statistical Computing. 2015;

[25] RStudio. Take Control of Your R Code. 2015;.

[26] Backstein G, GmbH R. Handbook on weaponry. Dsseldorf: Rheinmetall; 1982. 


\section{Appendix A: Pressure Trace Comparisons}




\section{Special Comparison- 4.1 Grains Bullseye}

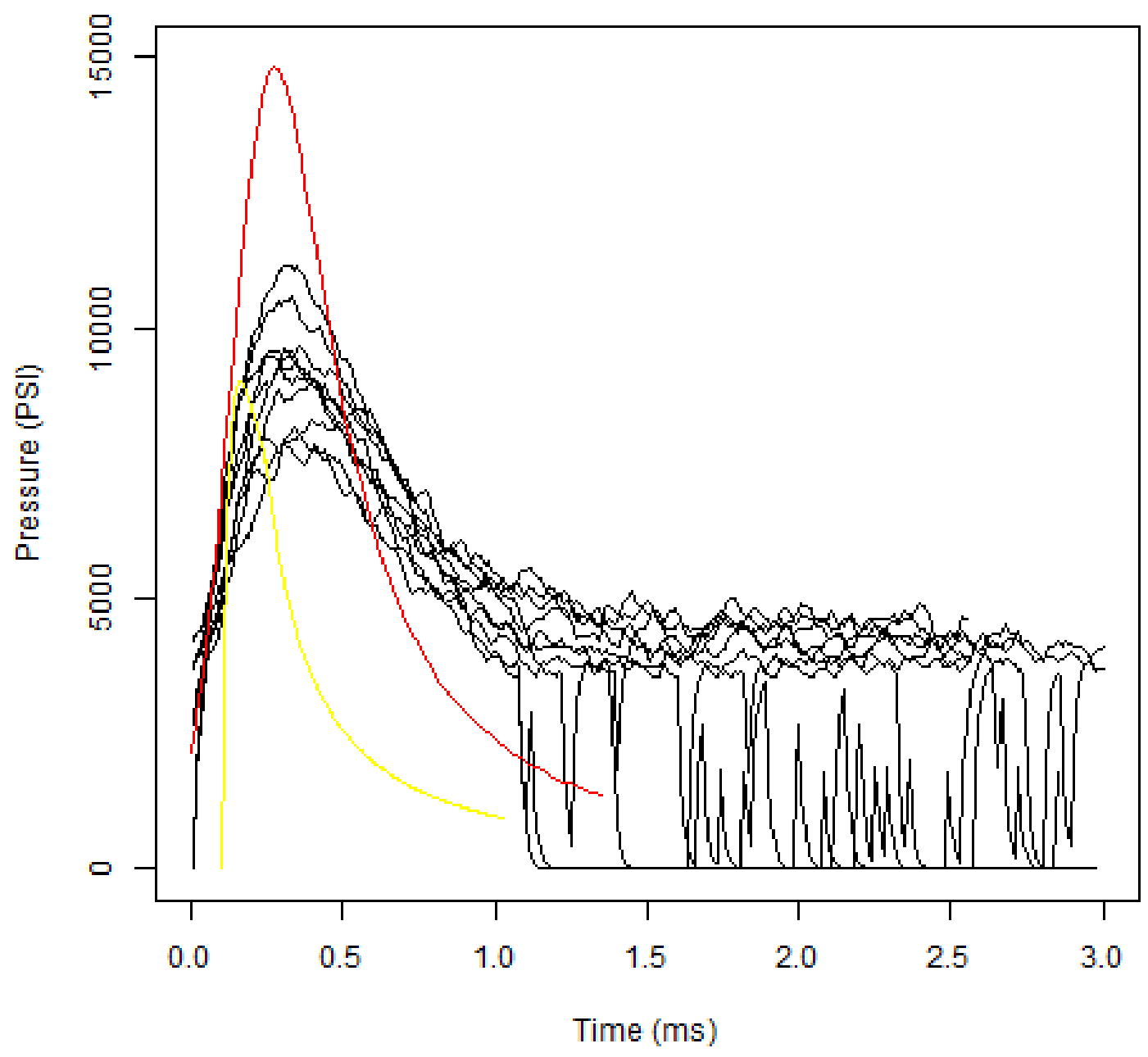

Figure 7.1: Pressure Trace graph showing the comparison of Quickload(red) trace, $\mathrm{R}$ script (yellow) trace, and traces obtained from firing (black) for .38 Special loaded with 4.1 grains of Bullseye powder. 
38 Special Comparison- 4.8 Grains Bullseye

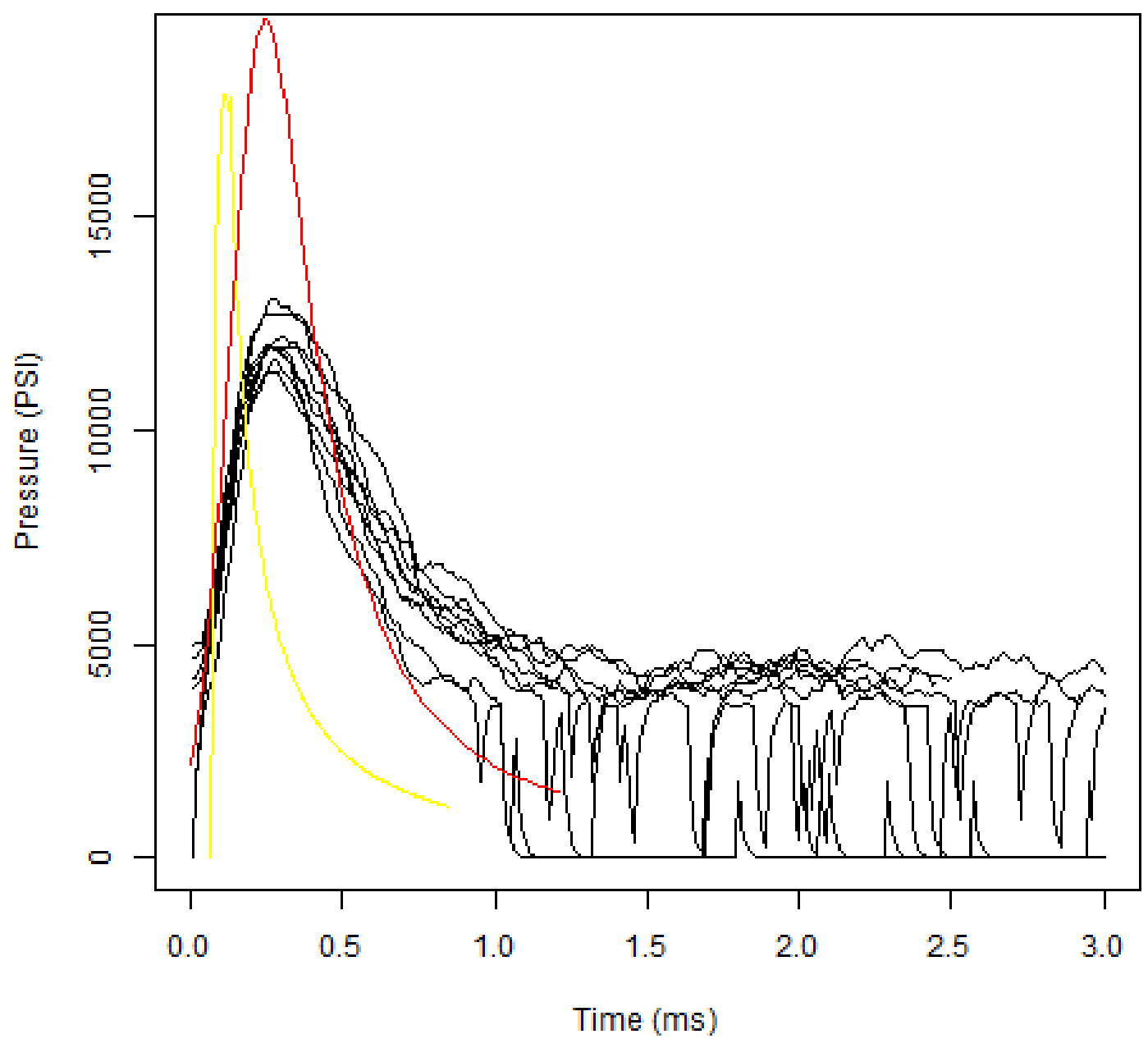

Figure 7.2: Pressure Trace graph showing the comparison of Quickload(red) trace, $\mathrm{R}$ script (yellow) trace, and traces obtained from firing (black) for .38 Special loaded with 4.8 grains of Bullseye powder. 


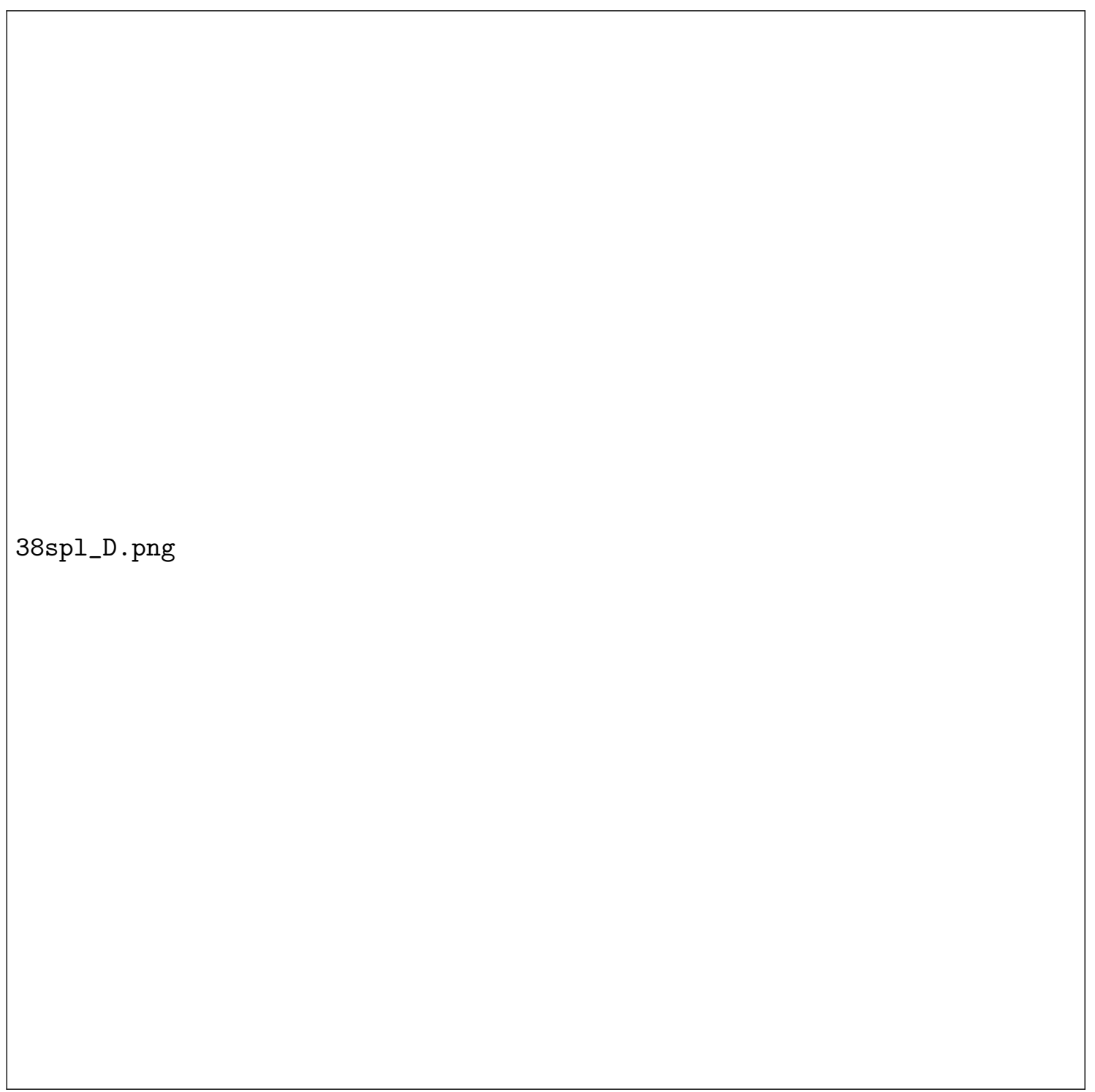

Figure 7.3: Pressure Trace graph showing the comparison of Quickload(red) trace, $\mathrm{R}$ script (yellow) trace, and traces obtained from firing (black) for a .38 Special loaded with 7.4 grains of Blue Dot powder. 


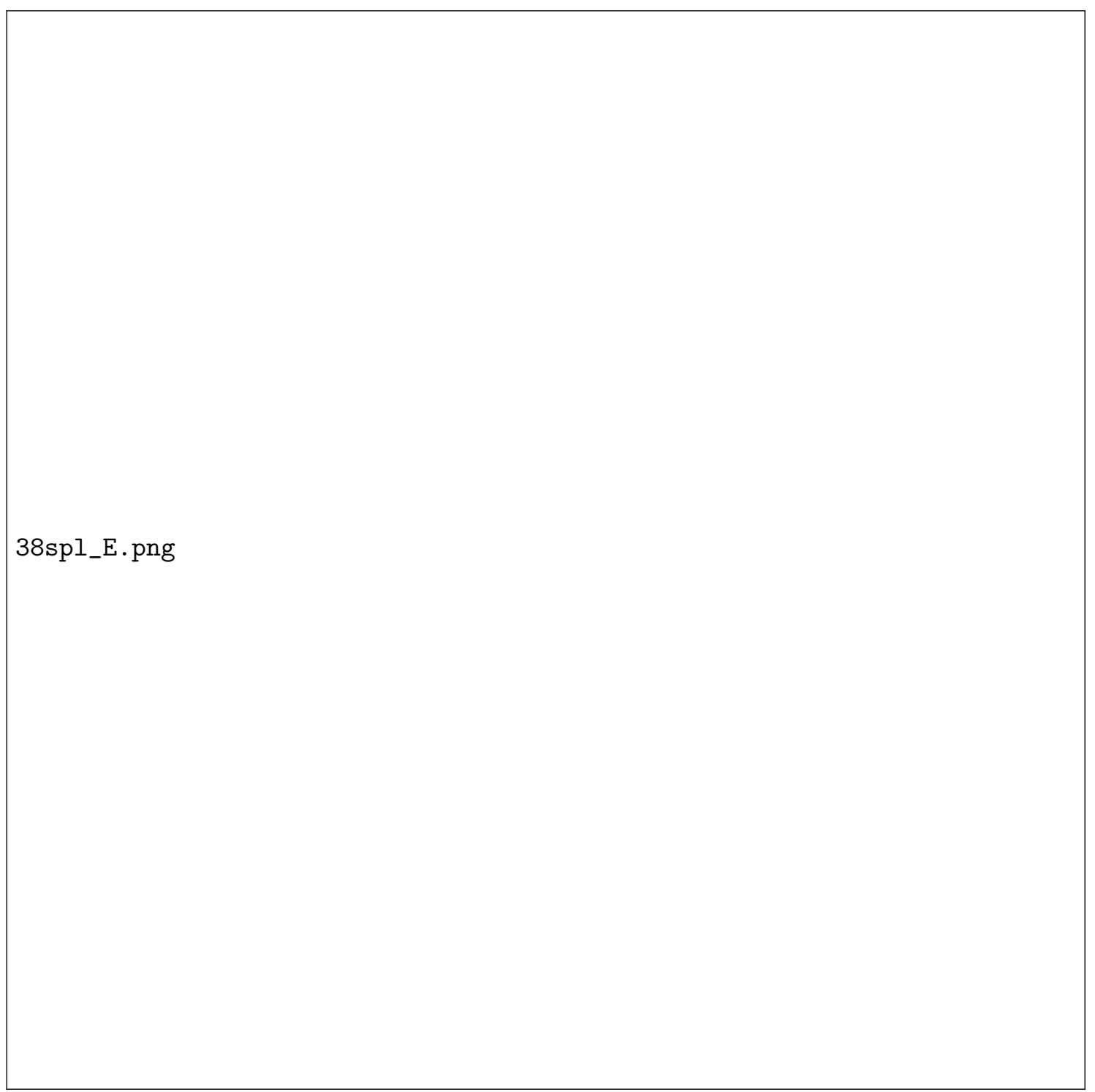

Figure 7.4: Pressure Trace graph showing the comparison of Quickload(red) trace, $\mathrm{R}$ script (yellow) trace, and traces obtained from firing (black) for a .38 Special loaded with 7.8 grains of Blue Dot powder. 


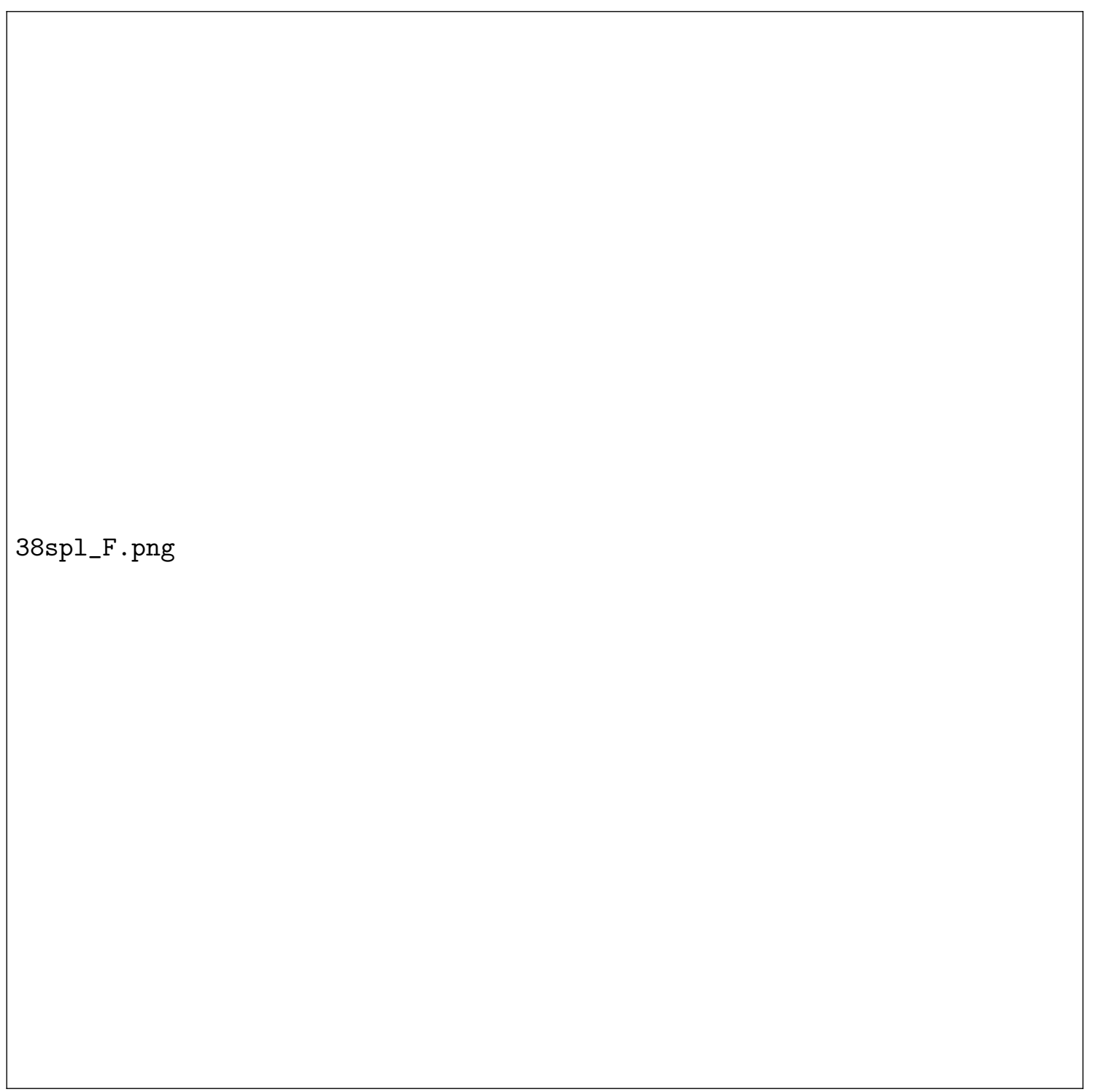

Figure 7.5: Pressure Trace graph showing the comparison of Quickload(red) trace, $\mathrm{R}$ script (yellow) trace, and traces obtained from firing (black) for a .38 Speical loaded with 8.2 grains of Blue Dot powder. 


\section{Magnum Comparison- 6.2 Grains Bullseye}

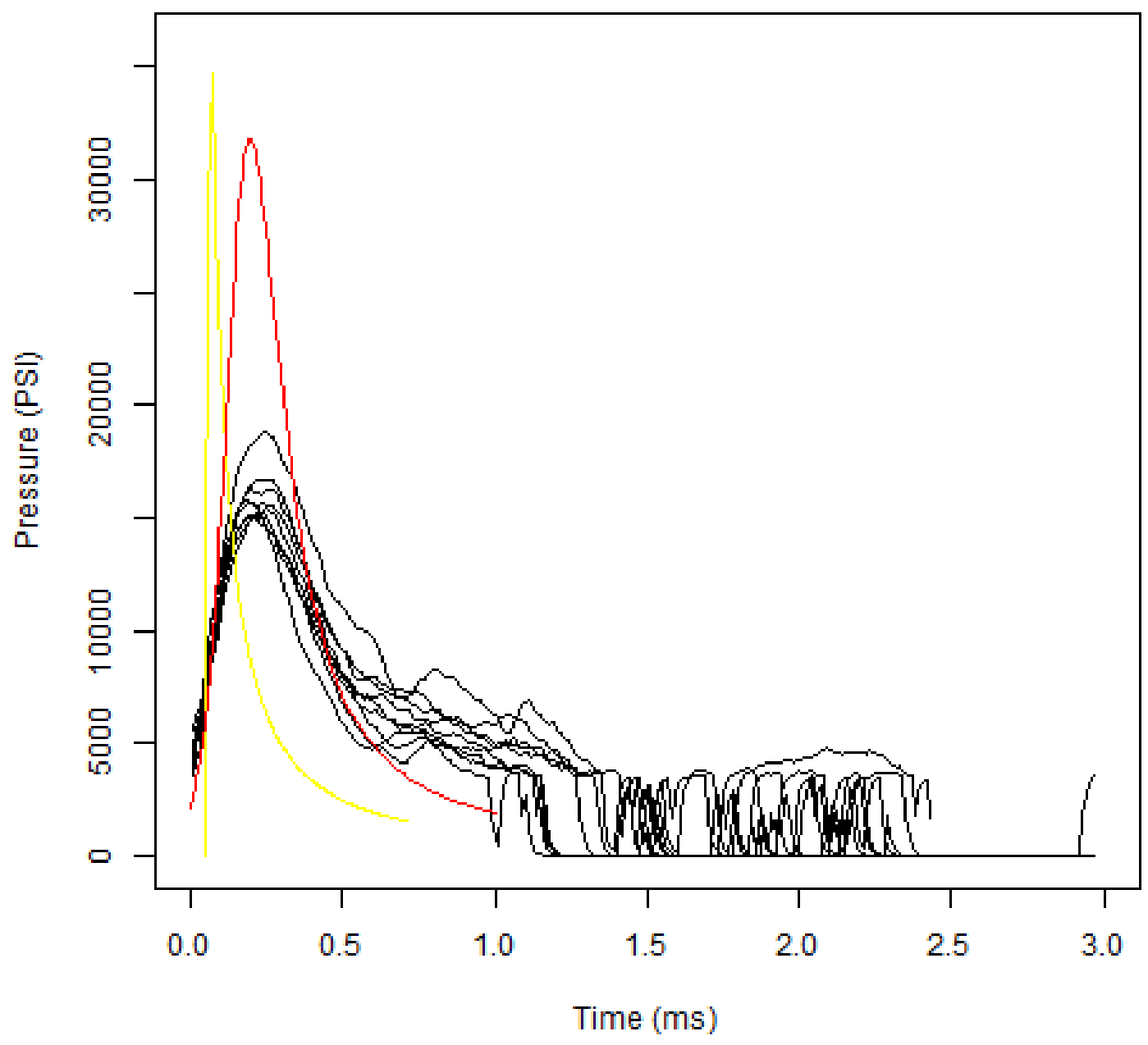

Figure 7.6: Pressure Trace graph showing the comparison of Quickload(red) trace, $\mathrm{R}$ script (yellow) trace, and traces obtained from firing (black) for a .357 Magnum loaded with 6.2 grains of Bullseye powder. 


\section{Magnum Comparison- 6.7 Grains Bullseye}

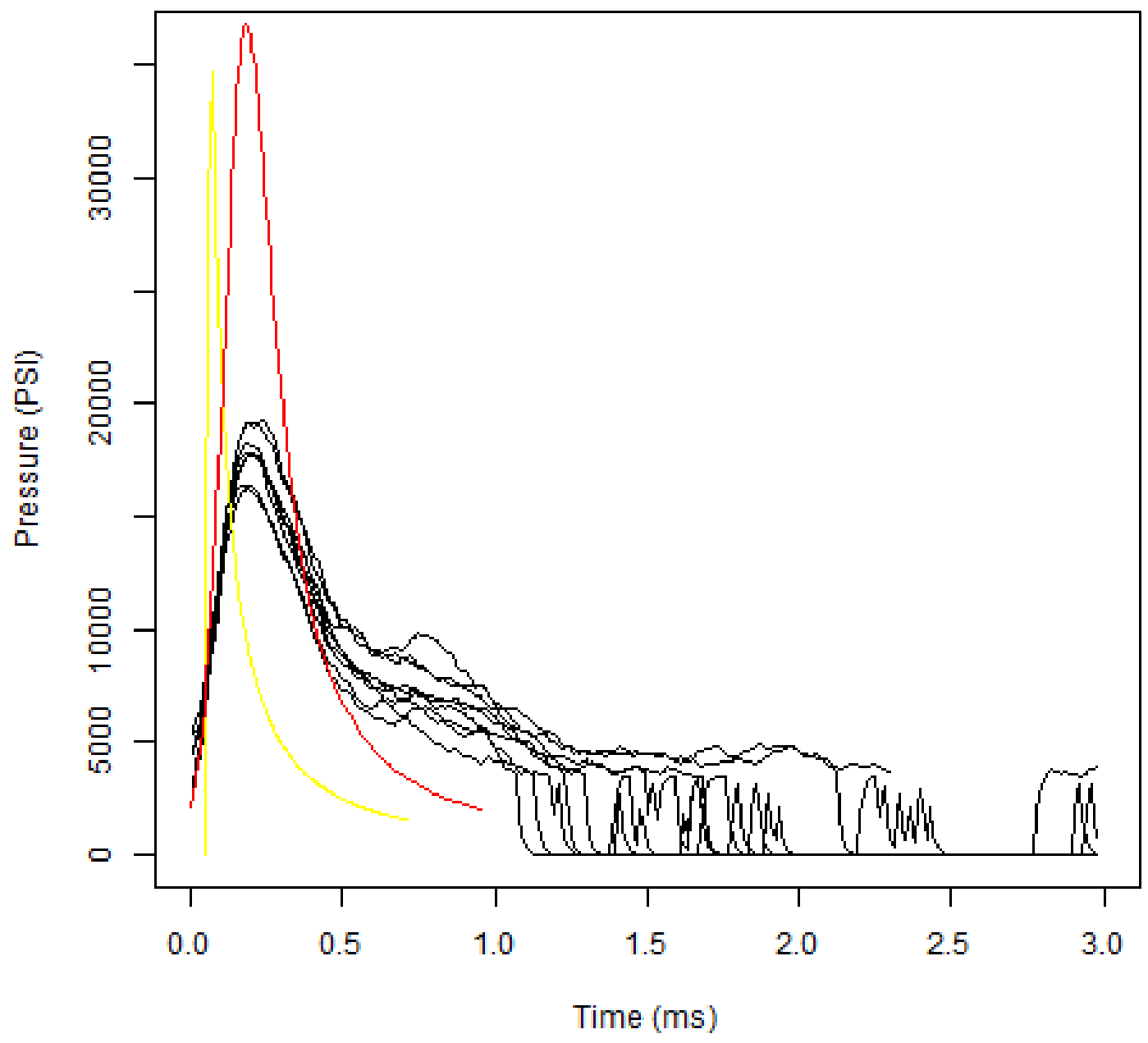

Figure 7.7: Pressure Trace graph showing the comparison of Quickload(red) trace, $\mathrm{R}$ script (yellow) trace, and traces obtained from firing (black) for a .357 Magnum loaded with 6.7 grains of Bullseye powder. 


\section{Magnum Comparison- 7.2 Grains Bullseye}

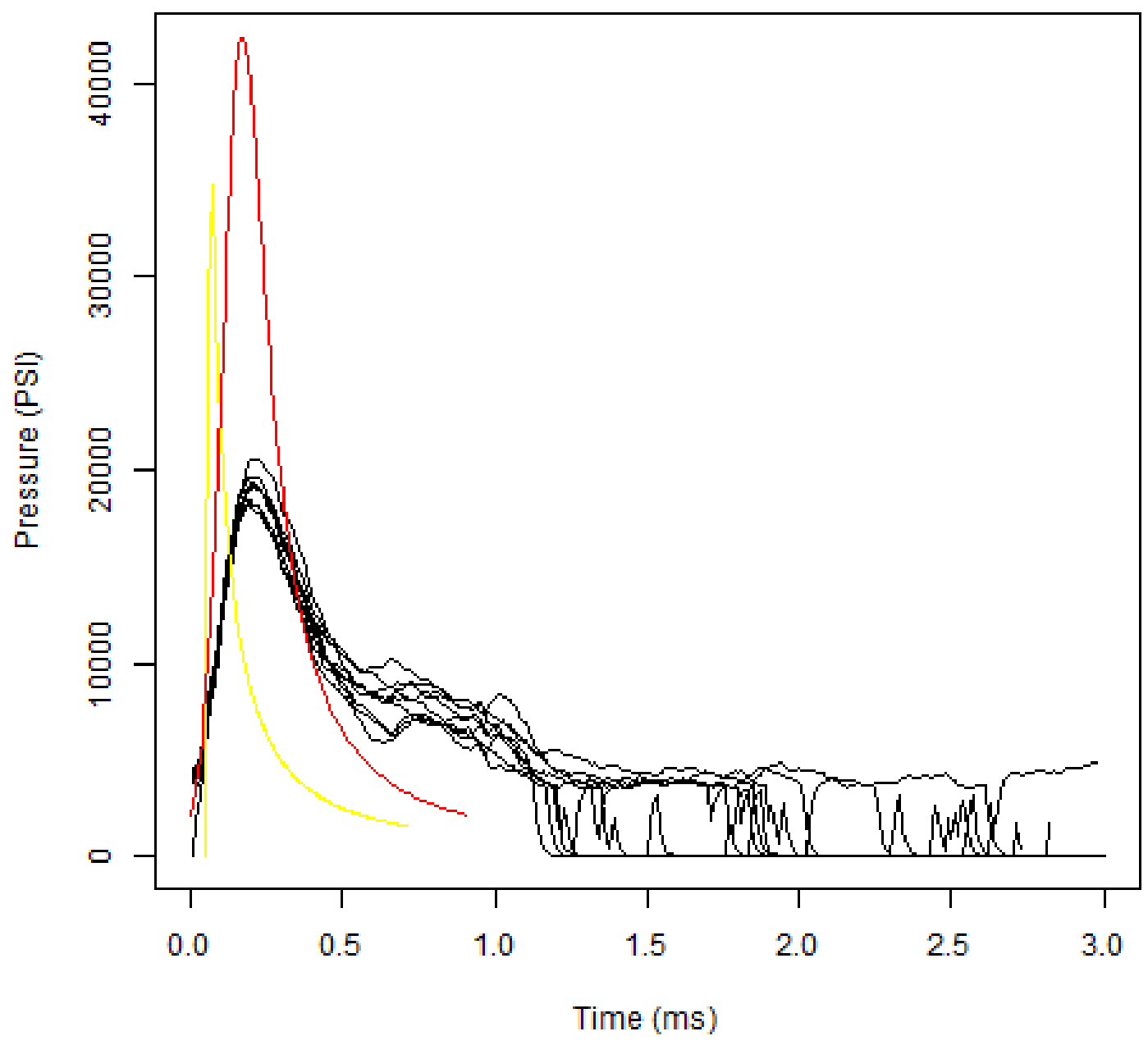

Figure 7.8: Pressure Trace graph showing the comparison of Quickload(red) trace, $\mathrm{R}$ script (yellow) trace, and traces obtained from firing (black) for a .357 Magnum loaded with 7.2 grains of Bullseye powder. 
357 Magnum Comparison- 11.6 Grains Bluedot

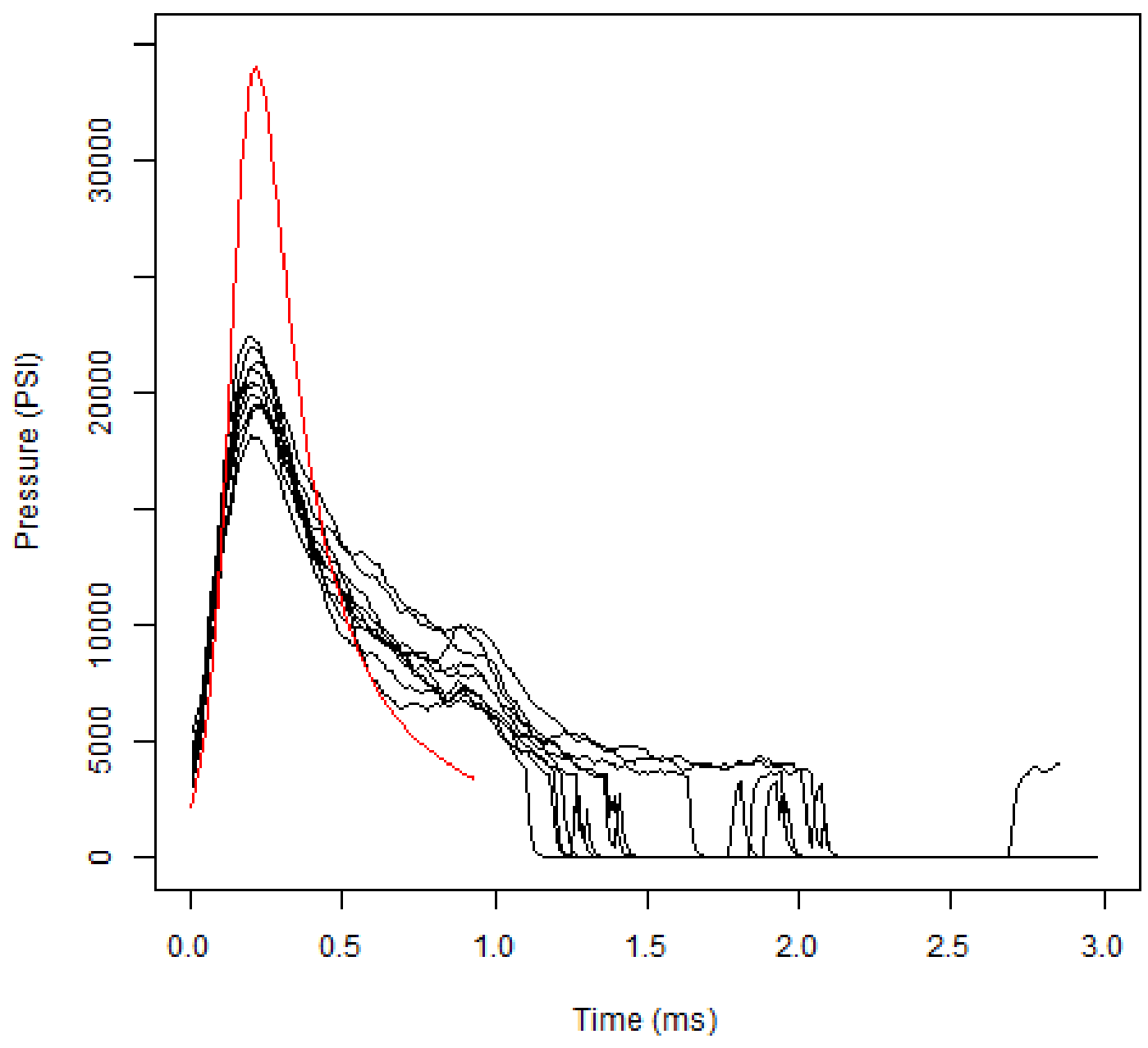

Figure 7.9: Pressure Trace graph showing the comparison of Quickload(red) trace, R script (yellow) trace, and traces obtained from firing (black) for a .357 Magnum loaded with 11.6 grains of Bluedot powder. 


\section{Magnum Comparison-12.0 Grains Bluedot}

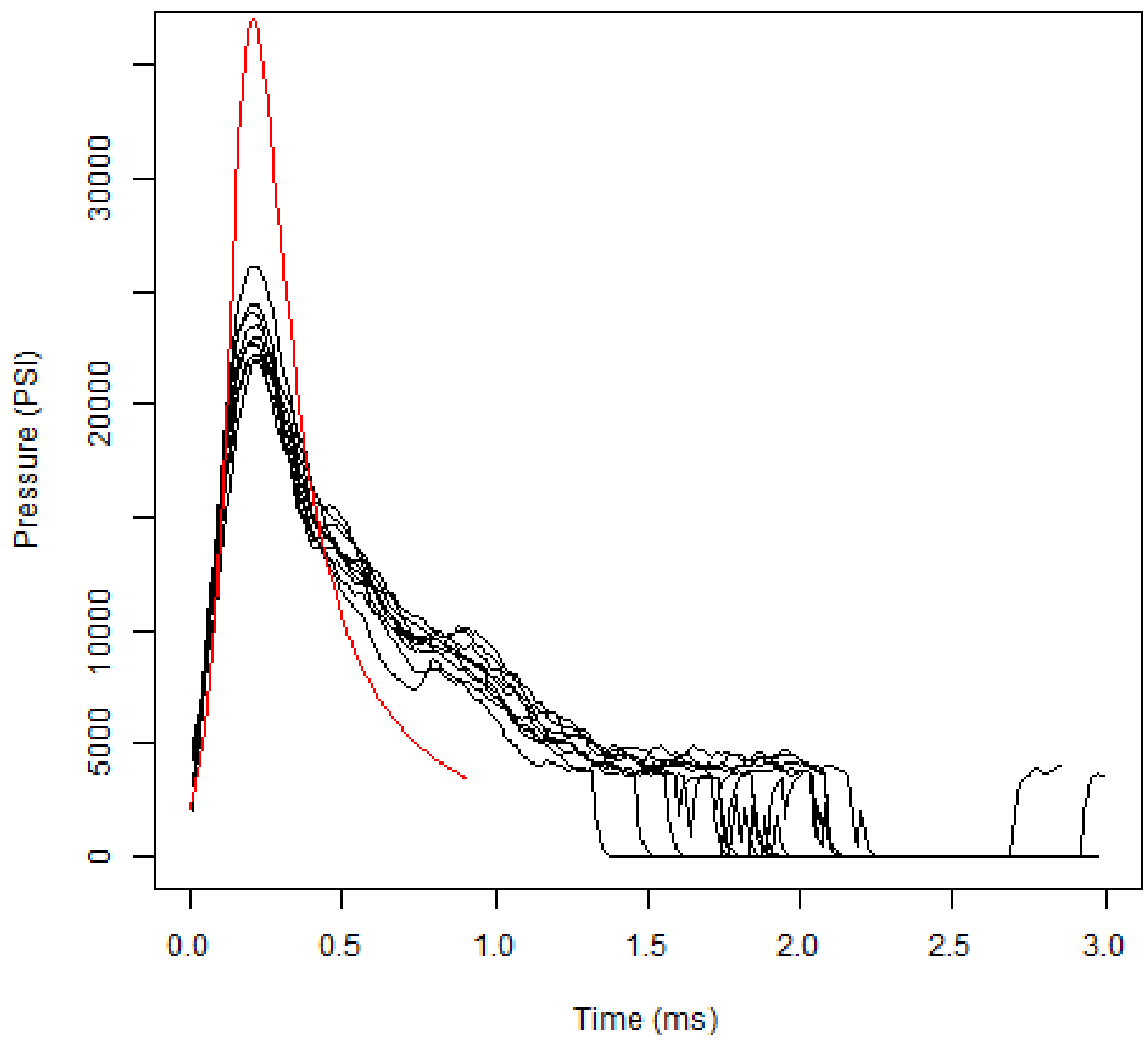

Figure 7.10: Pressure Trace graph showing the comparison of Quickload(red) trace, R script (yellow) trace, and traces obtained from firing (black) for a .357 Magnum loaded with 12.0 grains of Blue Dot powder. 
357 Magnum Comparison- 12.6 Grains Bluedot

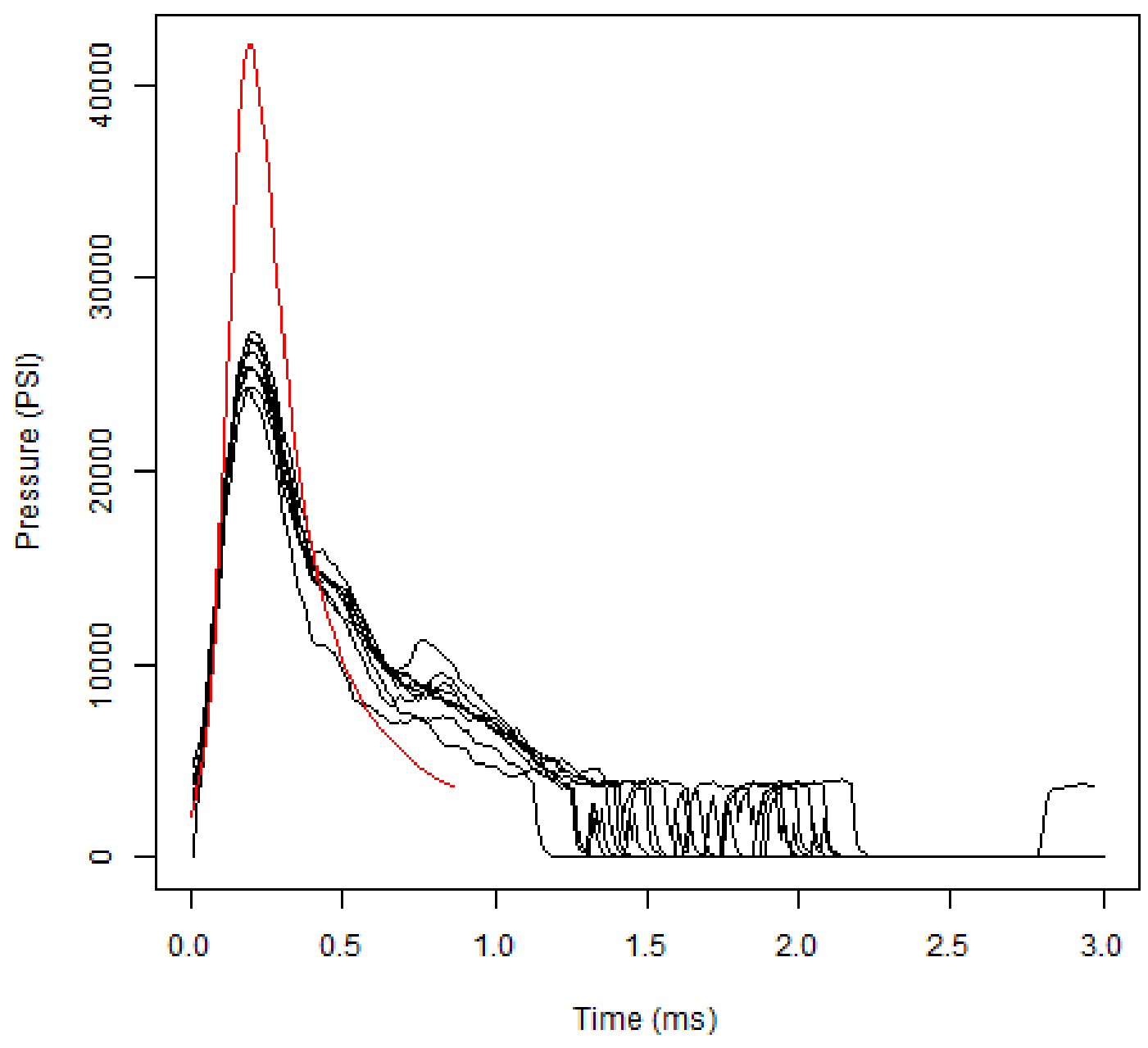

Figure 7.11: Pressure Trace graph showing the comparison of Quickload(red) trace, R script (yellow) trace, and traces obtained from firing (black) for a .357 Magnum loaded with 12.6 grains of Blue Dot powder. 


\section{Appendix B: R Scripts}

\subsection{Handbook on Weaponry Internal Ballistics Estimation}

\# \#\# A: Interior ballistics data

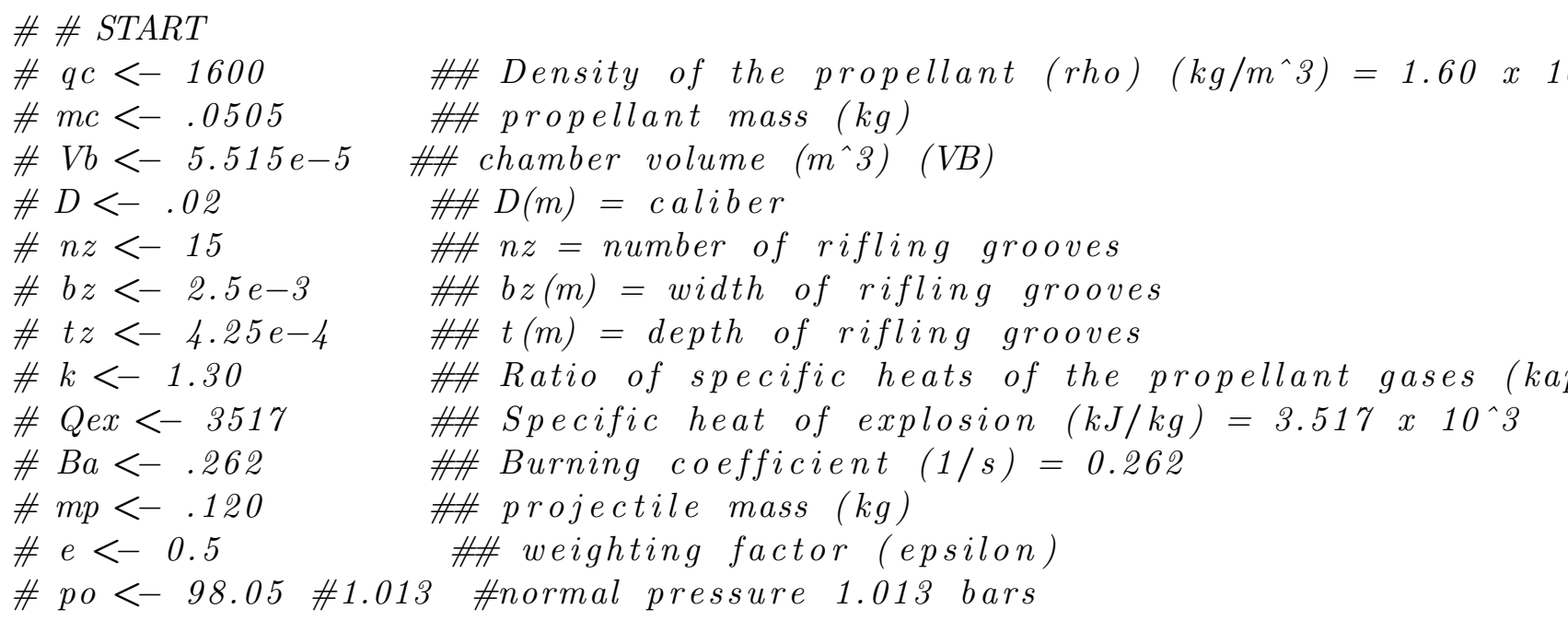

\#\# START

$\mathrm{qc}<-1630$

\# Density of the propellant (rho) $\left(\mathrm{kg} / \mathrm{m}^{\wedge} 3\right)=1.60 \times 10^{\wedge}$ $\mathrm{mc}<-0.22 / 1000$ \#\# propellant mass ( $\mathrm{kg})$

$\mathrm{Vb}<-1 \mathrm{e}-6 \quad$ \# chamber volume ( $\left.m^{\wedge} 3\right)$ (VB)

$\mathbf{D}<-.357 * 25.4 / 1000 \quad$ \#\# $D(m)=$ caliber

$\mathrm{nz}<-6 \quad$ \#\# $n z=$ number of rifling grooves

bz $<-2.3 \mathrm{e}-3$

\#\#z $(m)=$ width of rifling grooves

$\mathrm{tz}<-3 \mathrm{e}-4$ \#\# $t(m)=$ depth of rifling grooves

$\mathrm{k}<-1.209$

Qex $<-5158$ \#\# Ratio of specific heats of the propellant gases (kap. \#\# Specific heat of explosion $(\mathrm{kJ} / \mathrm{kg})=3.517 \times 10^{\wedge} 3$ 


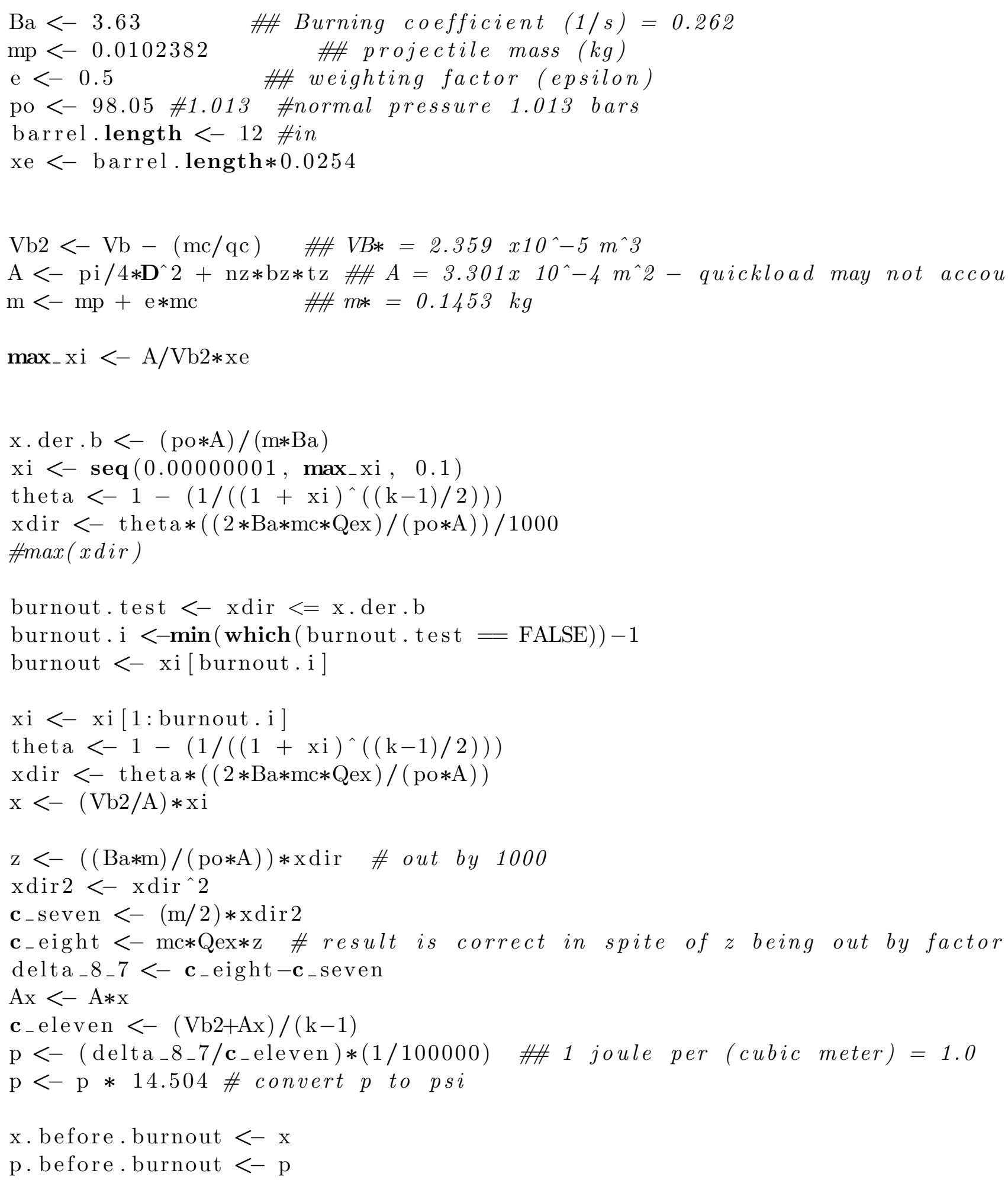


t. before. burnout $<-\mathrm{x} / \mathrm{xdir}$

$\# p l o t(x, p$, type $=" n ", x \lim =c(0,1.8), \quad y \lim =c(0,10000), \quad x l a b="$ distance, yla lines (t. before . burnout, p. before.burnout, col="green")

\#plot(times, $p$, type $=" l ", x \lim =c(0,0.003), y \lim =c(0,10000)$, xlab="time,$y$ \#plot(x.before.burnout, p.before.burnout, type="l")

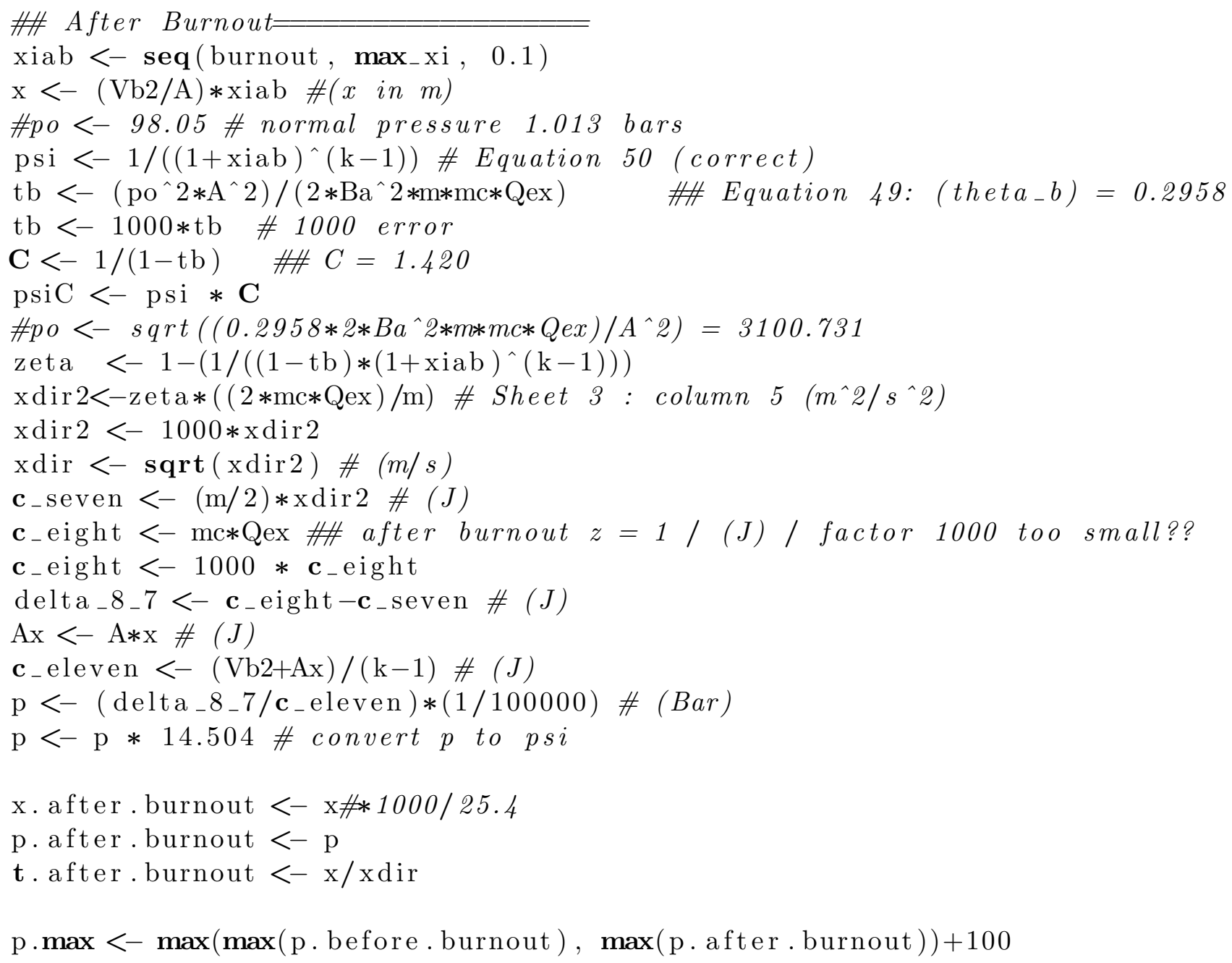


t. complete $<-\mathbf{c}(\mathbf{t}$. before.burnout, t.after.burnout)

plot (x.complete, p.complete, type=" l", $x \lim =\mathbf{c}(0,12), \quad y \lim =\mathbf{c}(0, p \cdot \max ), \quad x \operatorname{lab}$ ylab="pressure $\lrcorner($ psi $) ")$

plot (t.complete $* 1000$, p.complete, type="l", $x \lim =\mathbf{c}(0,3), \quad y \lim =\mathbf{c}(0,11000)$, ylab="pressure $\left.\_(\text {psi }) "\right)$

$\#$

$\#$ newt $<-(t$. complete $/ \max (t$. complete $)) * \max \left(Q L \$ t_{-} m s\right)$

$\#$

\#

\# plot(newt, p.complete, type $=" l ", x \lim =c(0,3), \quad y \lim =c(0,11000), \quad x l a b=" t$ \# ylab="pressure (psi)")

\#lines (t.complete*1000, p.complete, col="yellow")

\# main.dir <- "Z:/James Hamilton/PressureTrace/Traces/CSV2/Output"

$\#$

\# file_name_back <-"38sp_02_Shot5"

\# file_name $<-$ "38sp_02_Shotr"

$\#$

\# fname <-paste0(main.dir," /", file_name,".csv")

\# shotter $<-$ read.csv(fname)

$\#$

\# fname <- paste0(main.dir,"/", file_name_back,".csv")

\# background $<-$ read.csv(fname)

$\#$

$\#$

\# shotter\$time $<-$ shotter $\$$ time* 1000

$\#$

\# pressures <- shotter $\$$ pressure-background $\$$ pressure

lines ( shotter \$time, shotter \$pressure, col=" blue")

abline $(\mathrm{h}=4000$, lt $\mathrm{y}=$ 'dashed ')

\# QL_name $<-"$ testlod"

\# old.dir <- "Z:/James Hamilton/PressureTrace/Traces/CSV/Output"

\# fname <- paste0(old.dir,"/", QL_name,".csv")

\# $Q L<-$ read.csv(fname)

\# names $(Q L)$ 
lines $\left(\mathrm{QL} \$ \mathbf{t}_{-} \mathrm{ms}, \mathrm{QL} \$ \mathrm{p}_{-}\right.$psi , col="green") $\max \left(\mathrm{QL} \$ \mathrm{t}_{-} \mathrm{ms}\right)$

\subsection{Plot Exported Quickload ${ }^{\mathrm{TM}}$ Data}

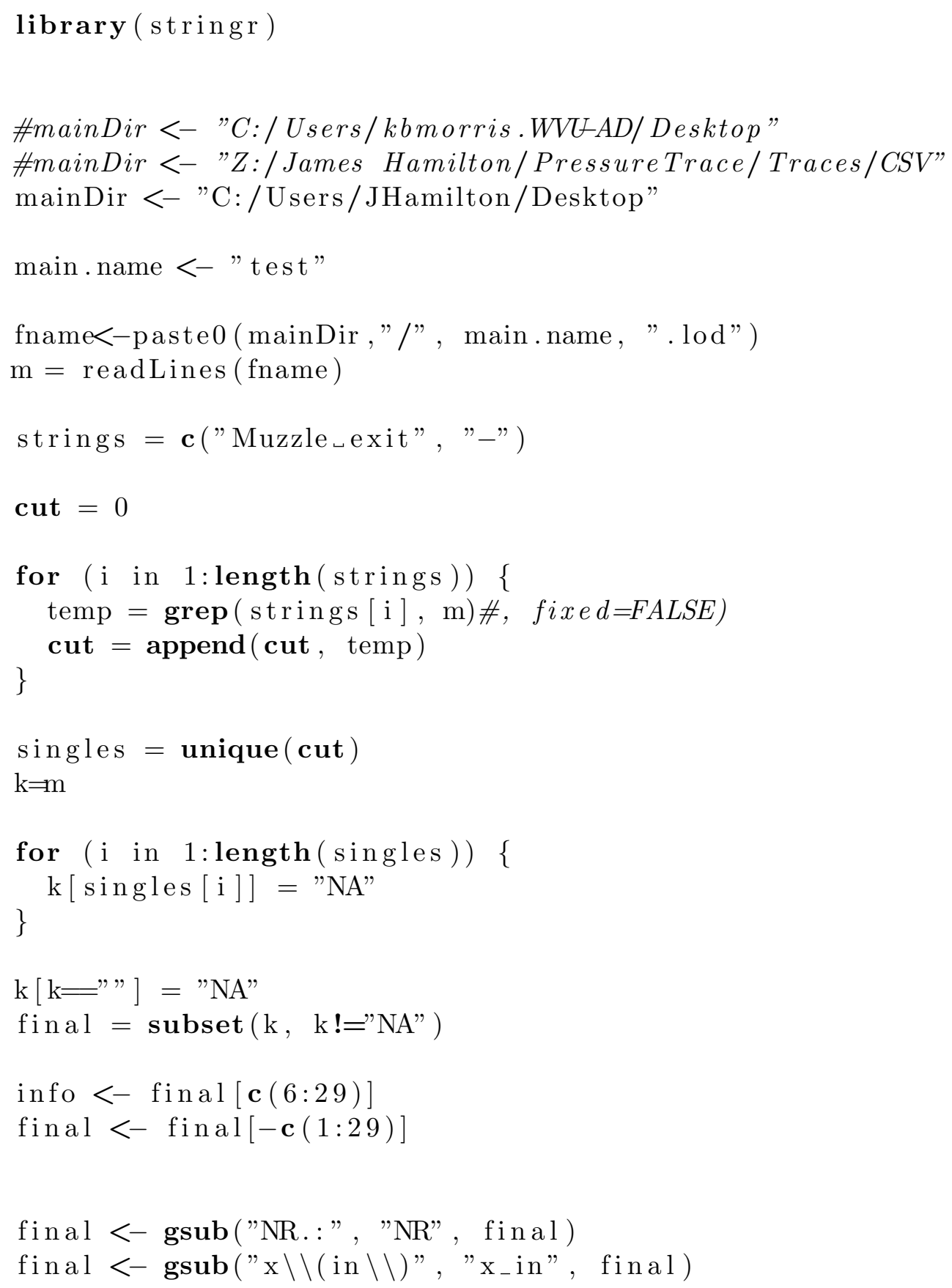




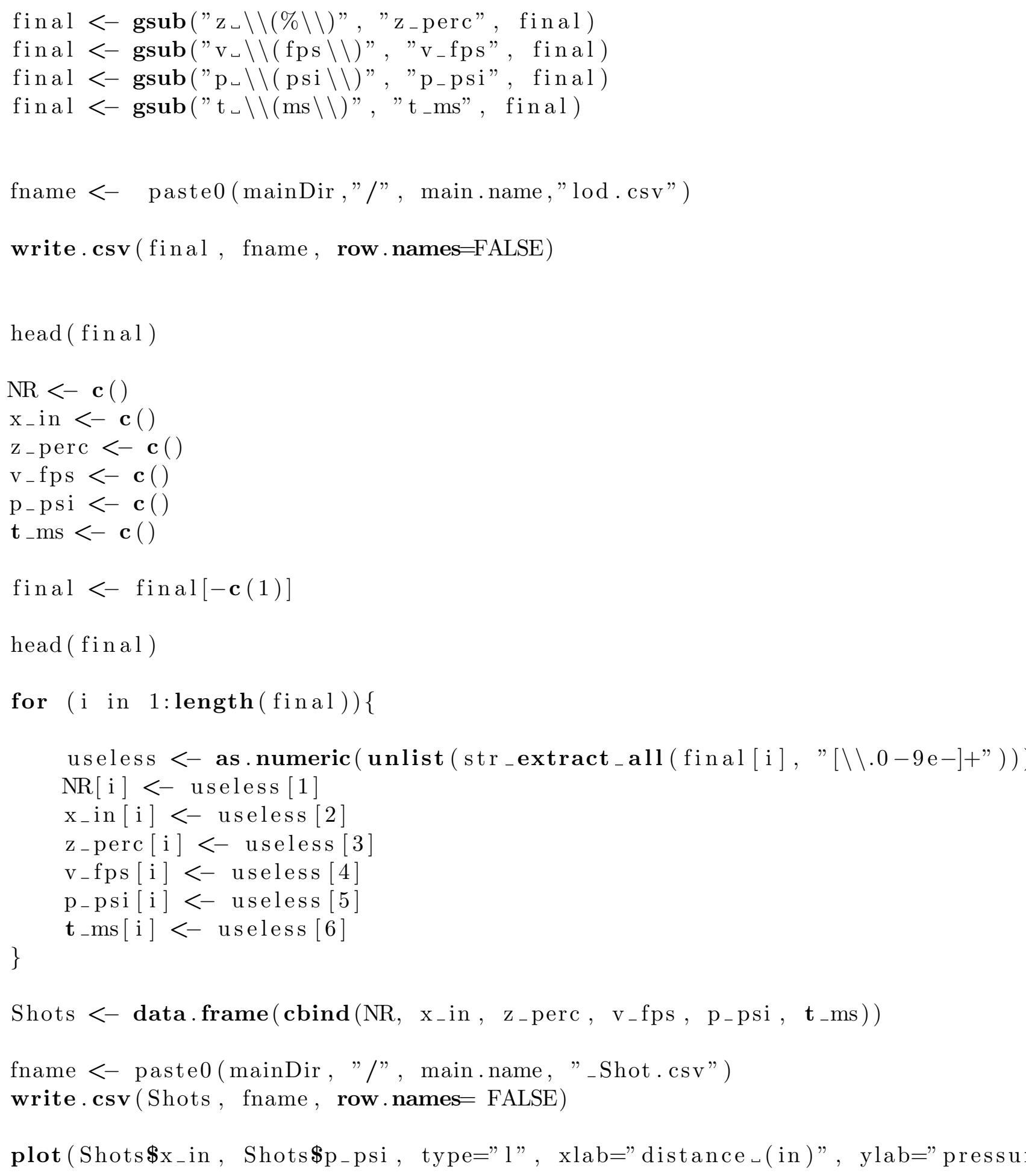


Propel $<-\operatorname{substr}($ info $[13]$, as numeric (nchar $("$ Propellant type $\ldots . . . . .=$ "

outs $<-$ regexpr("Load $\lrcorner$ density", info [14])

powder.weight $<-$ substr (info [14], as .numeric (nchar ("Charge ${ }$ weight

powder.weight <- as.numeric (gsub(" _",", , powder.weight))

cartridge $<-\operatorname{substr}($ info $[2]$, as . numeric $(\operatorname{nchar}("$ Cartridge $\ldots \ldots \ldots \ldots \ldots$

outs $2<-$ regexpr("Groove", info [5])

bullet.weight $<-$ substr (info [5], as numeric (nchar("Projectile ${ }_{-}$weight

bullet.weight <- as.numeric (gsub(" _",", , bullet.weight))

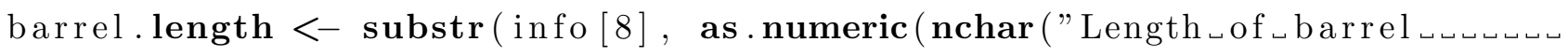

title $<-$ paste0 ("Chamber $\sqcup$ pressure: $"$ ", cartridge)

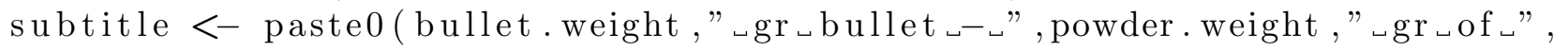

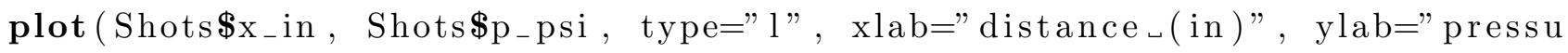
main $=$ title, $\mathbf{s u b}=$ subtitle,$\quad y \lim =\mathbf{c}\left(0, \max \left(\right.\right.$ Shots $\$ p_{-}$psi $\left.\left.)+100\right)\right)$

\subsection{Process Exported IBIS ${ }^{\circledR}$ data}

mainDir <- "Z:/James_Hamilton/IBIS /38_357_Data/"

\#info<-read.csv("Z:/Firearms/Firearms/Files - CSV and Excel/CSV/38Sp and 3

\#Pay attention to the file dates in the datafiles in info that are read in \#Change wider to how every many characters the date contains

\#For file RUG9 wider $=6$

\#wider<-2

filer $<-$ paste0 (mainDir,"CAT.csv")

info<-read.csv (filer)

FileStrings $<-$ info $\$$ Files

GunFile<-"CAT" 


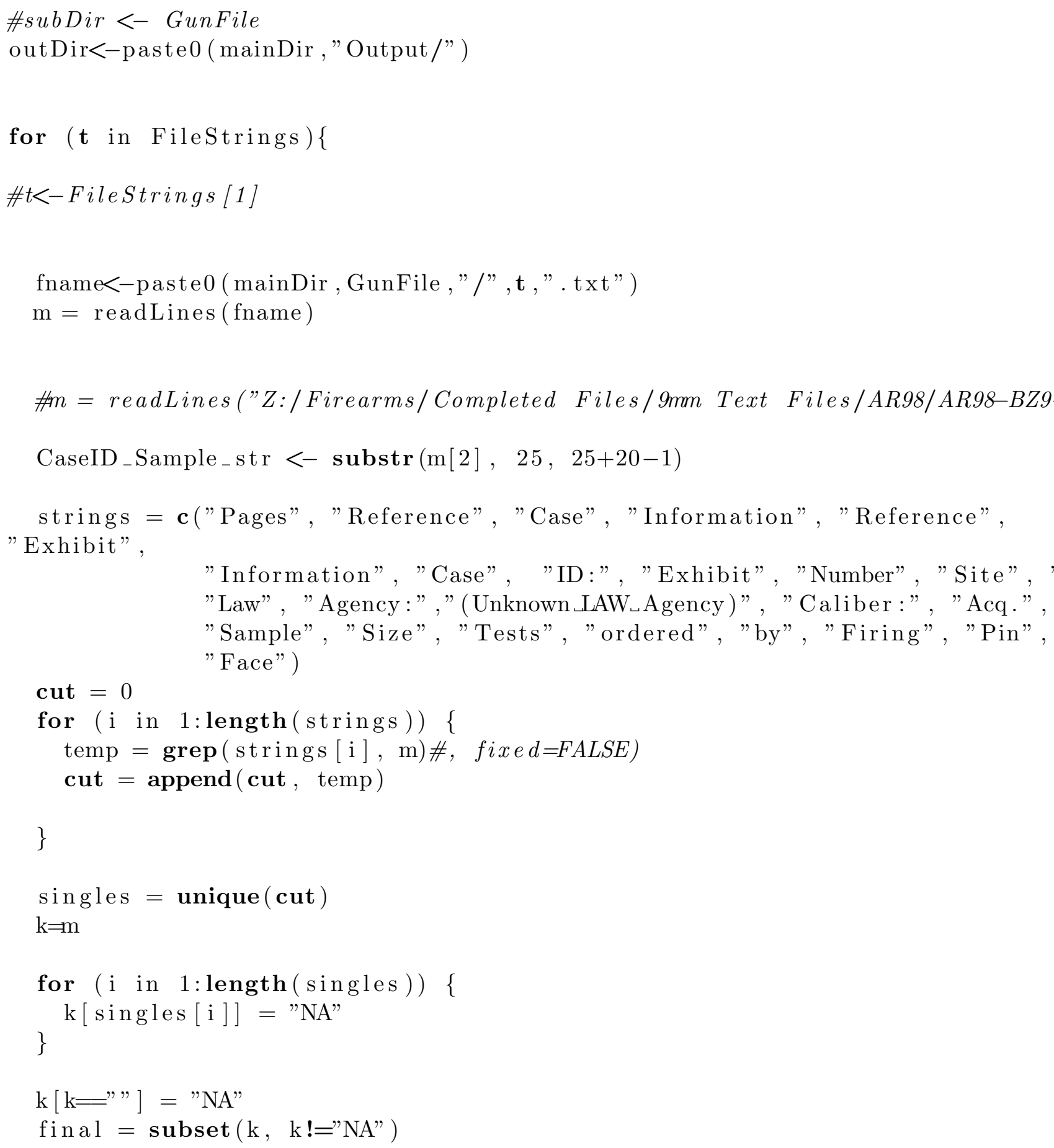




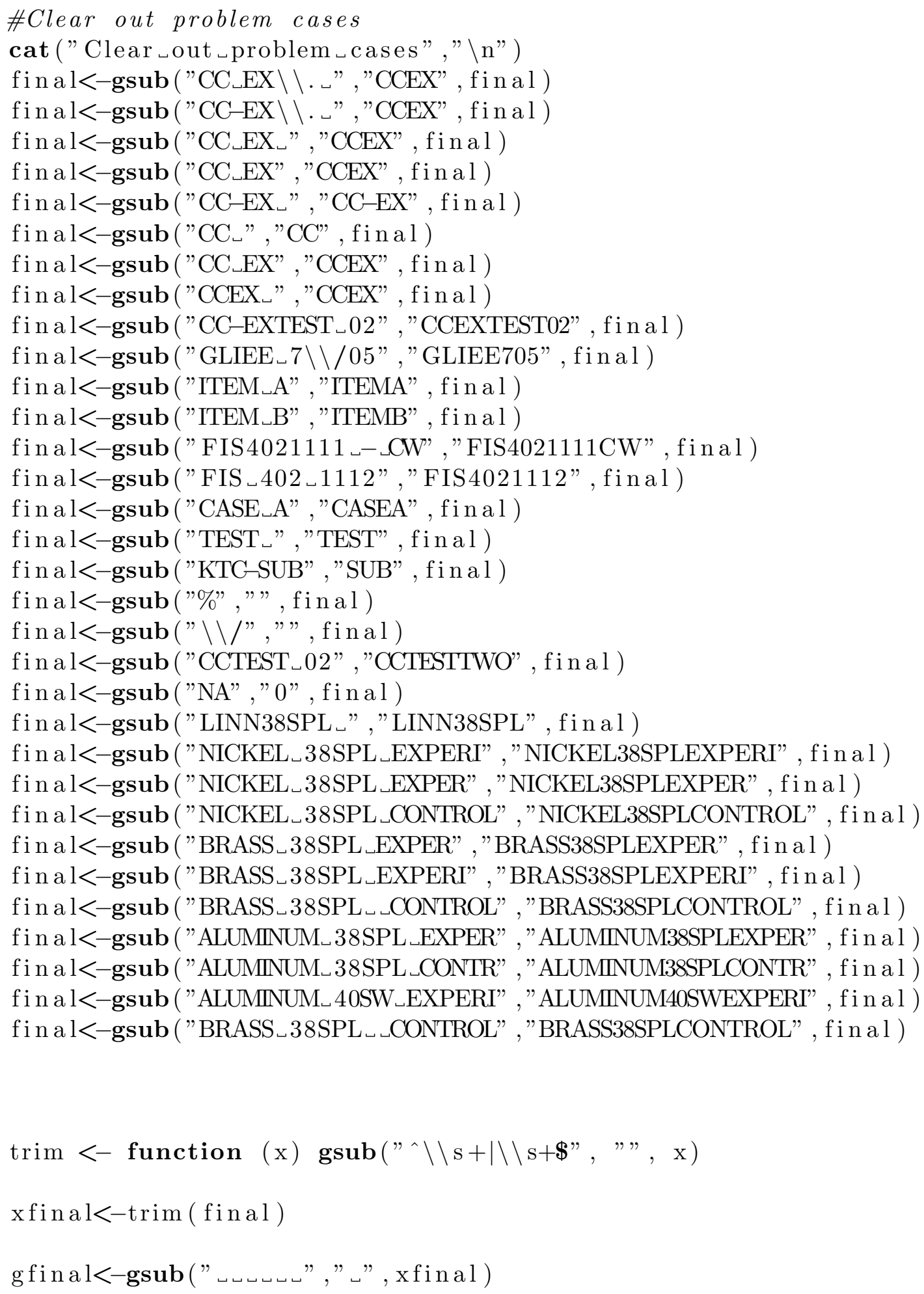




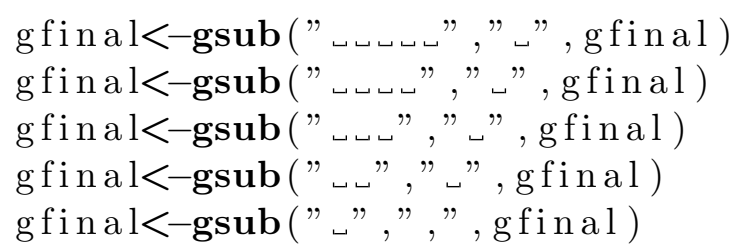




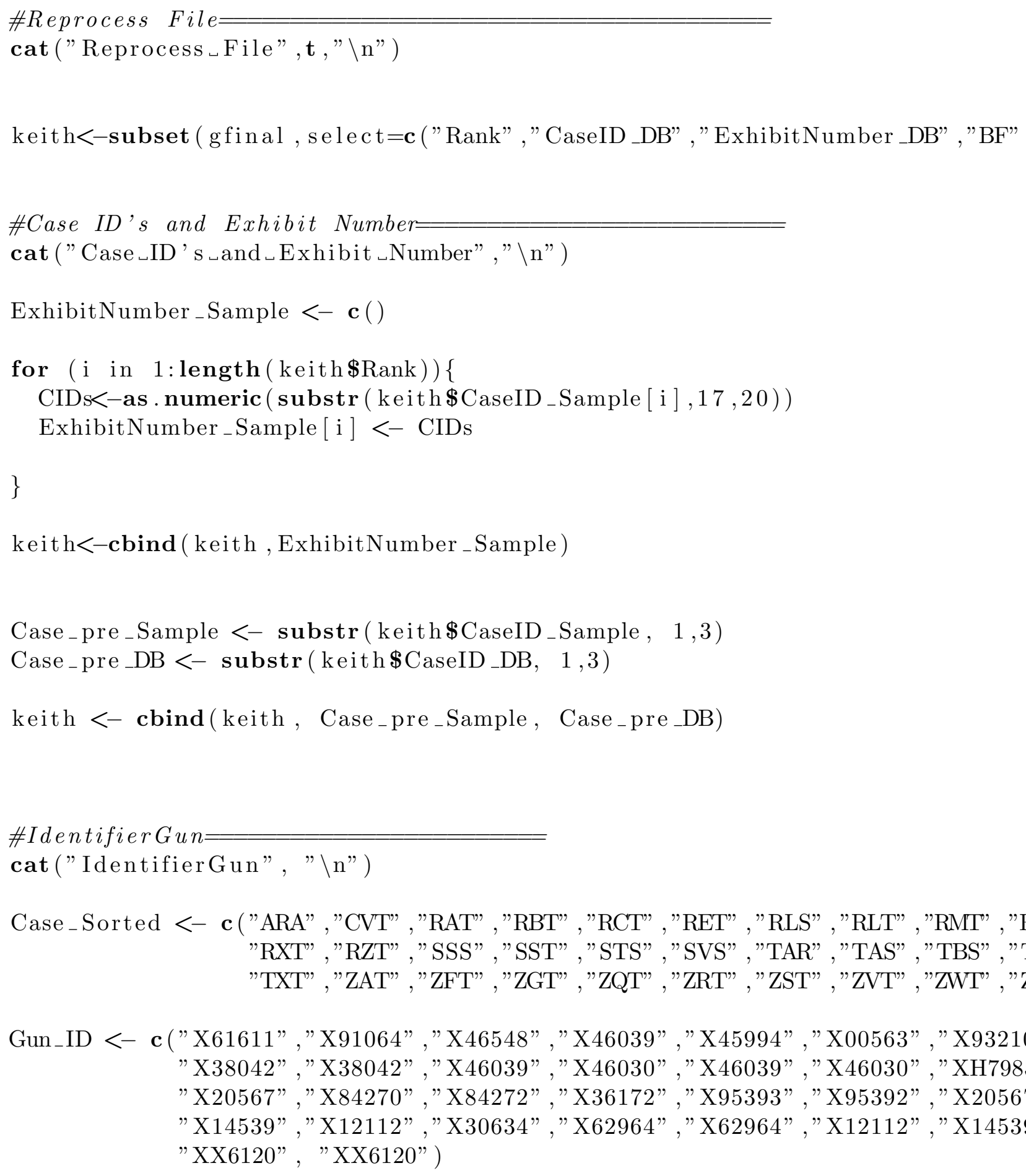




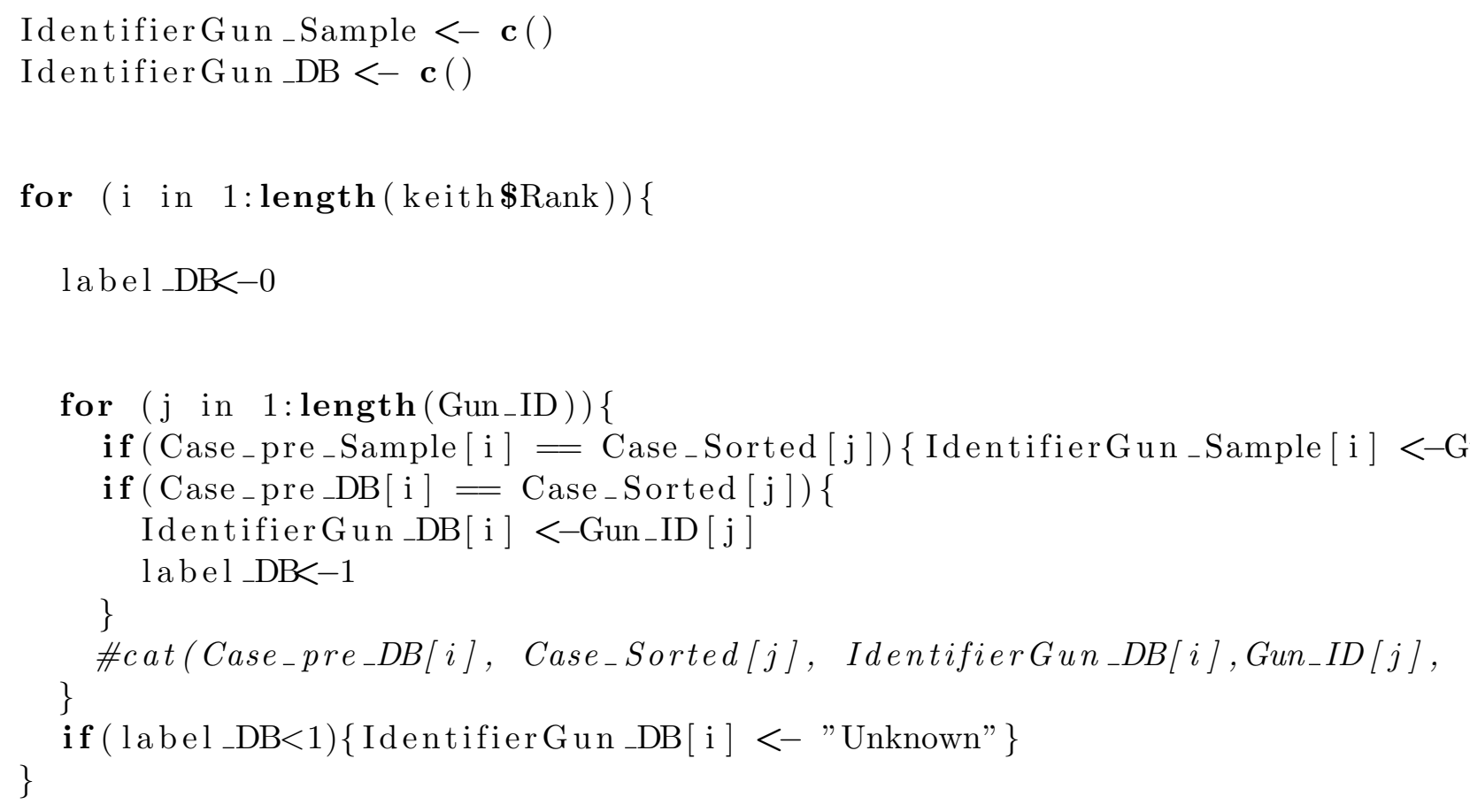


keith <- cbind (keith, Model_Sample, Make_Sample, Model_DB, Make_DB)

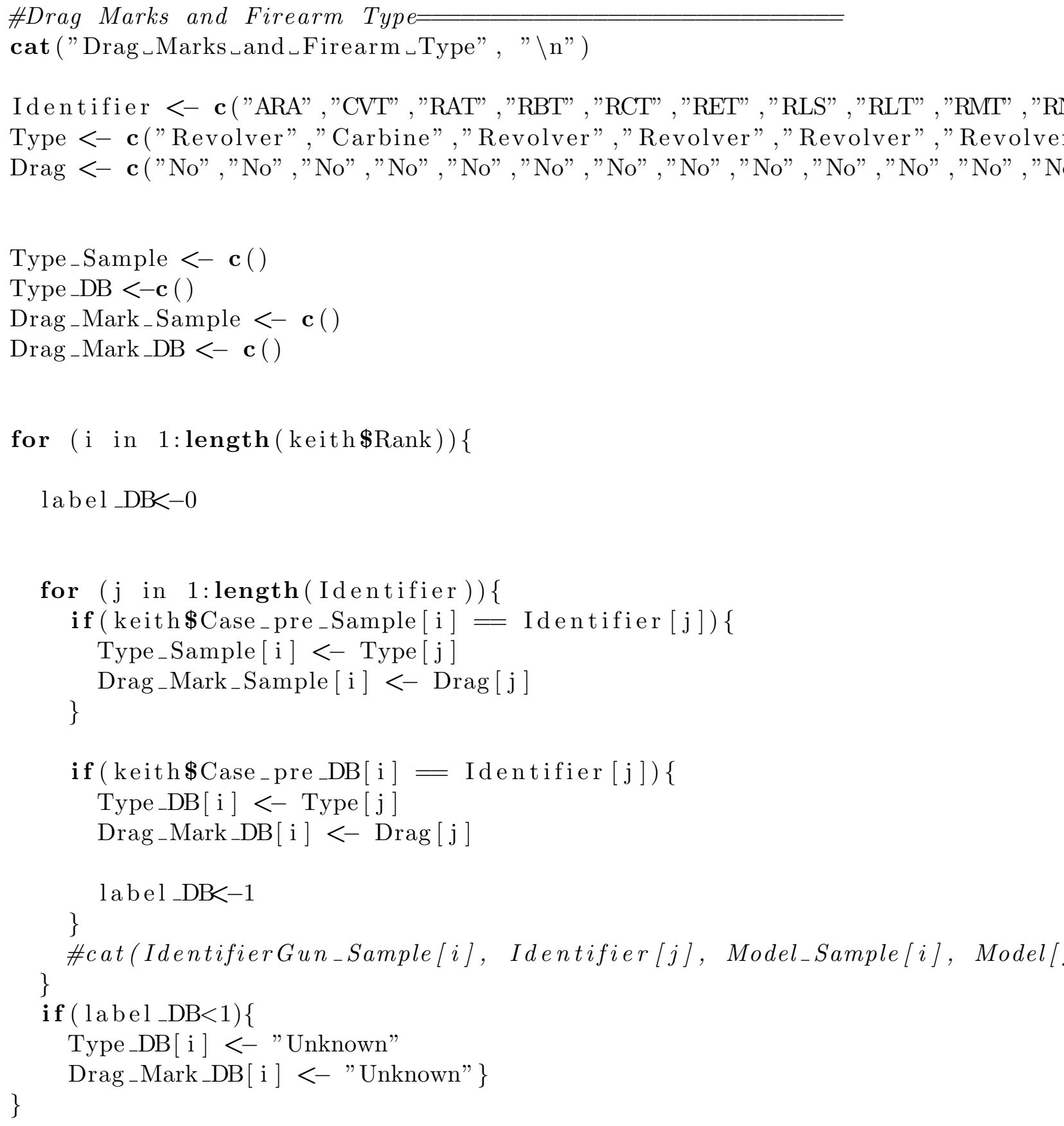


keith $<-$ cbind $($ keith, Type_Sample, Type_DB, Drag_Mark_Sample, Drag_Mark_I

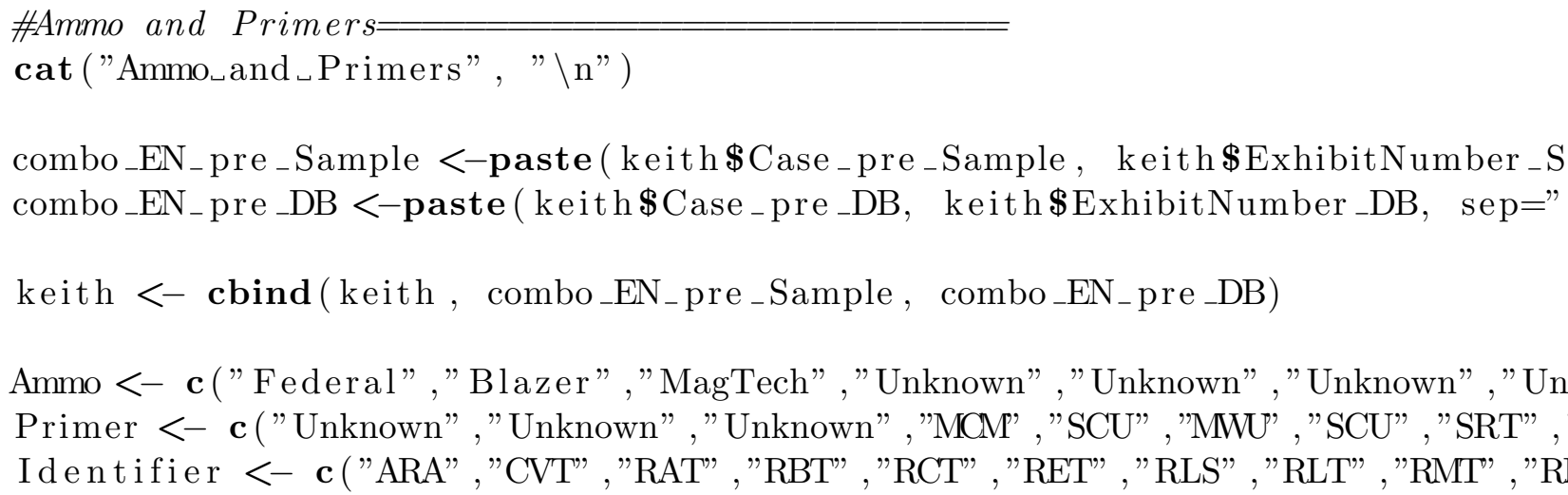

Ammo_Sample $<-\mathbf{c}()$

Ammo_DB $<-\mathbf{c}()$

Primer_Sample $<-\mathbf{c}()$

Primer $\_$DB $<-\mathbf{c}()$

for ( $i$ in $1:$ length $($ keith $\$$ Rank $))\{$

label $\_$DB< -0

for ( $\mathrm{j}$ in 1 : length(Identifier $))\{$

if (keith $\$$ Case_pre_Sample [i] $=$ Identifier $[\mathbf{j}])\{$

Ammo_Sample [i ] $<-$ Ammo[j]

Primer_Sample $[\mathrm{i}]<-$ Primer $[\mathrm{j}]$

\}

if (keith\$Case _ pre_DB $[\mathrm{i}]=$ Identifier $[\mathrm{j}])\{$

Ammo_DB $[\mathrm{i}]<-$ Ammo $[\mathrm{j}]$

Primer_DB $[\mathrm{i}]<-$ Primer $[\mathrm{j}]$

label_DB<-1

\} 


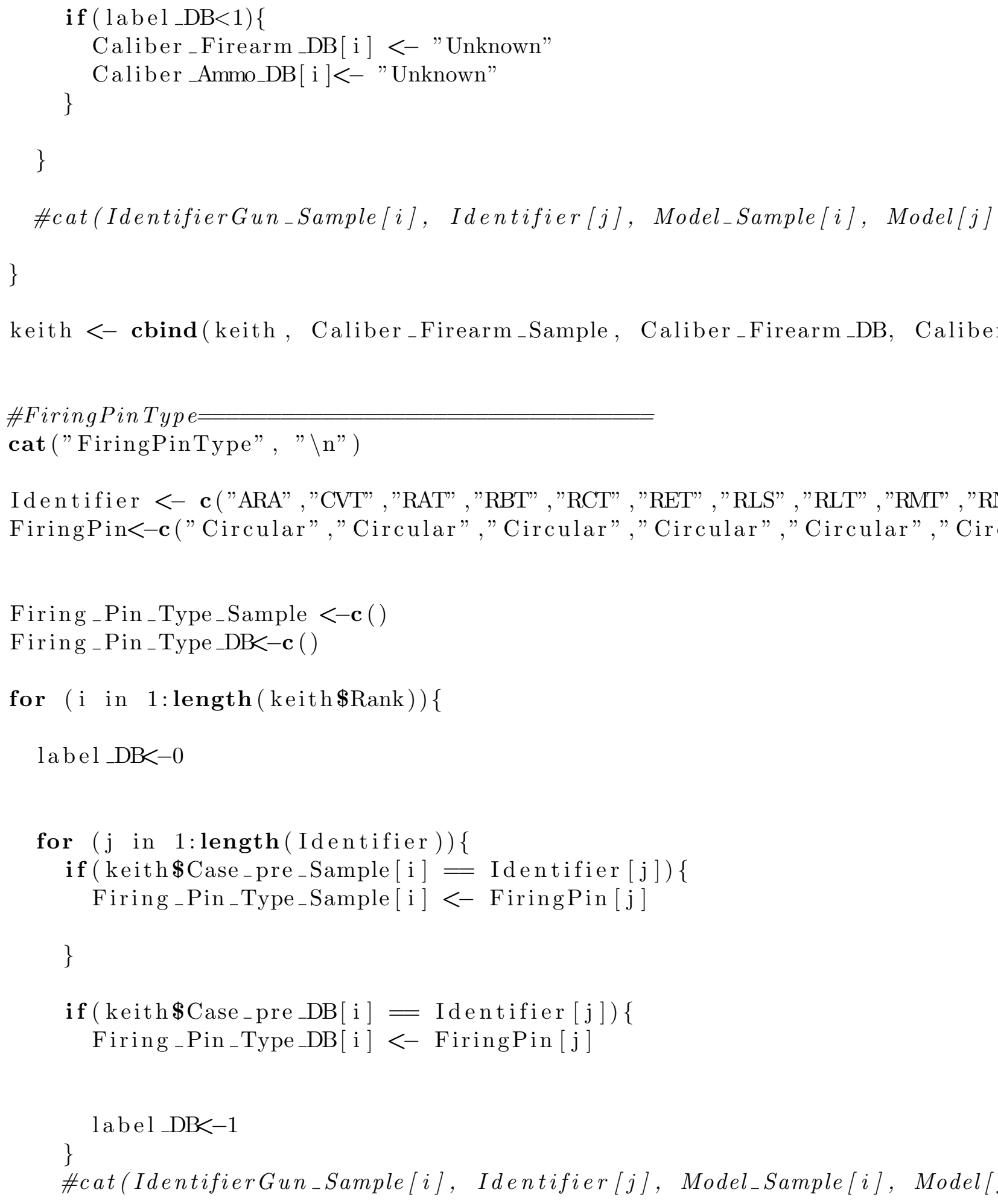




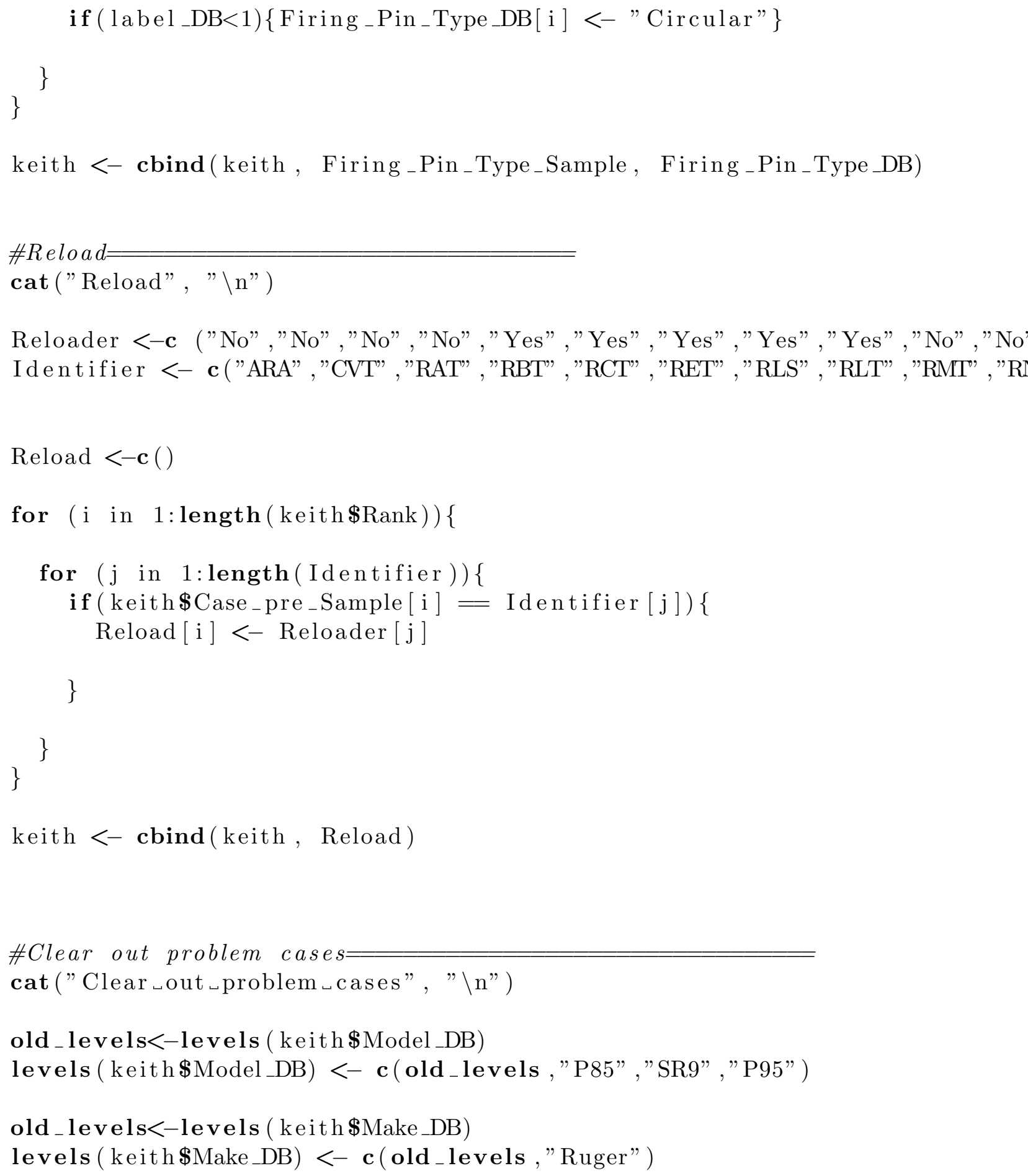


old _levels $<-$ levels ( keith $\$$ Drag_Mark_DB)

levels ( keith\$Drag_Mark_DB) <- c (old_levels, "Yes")

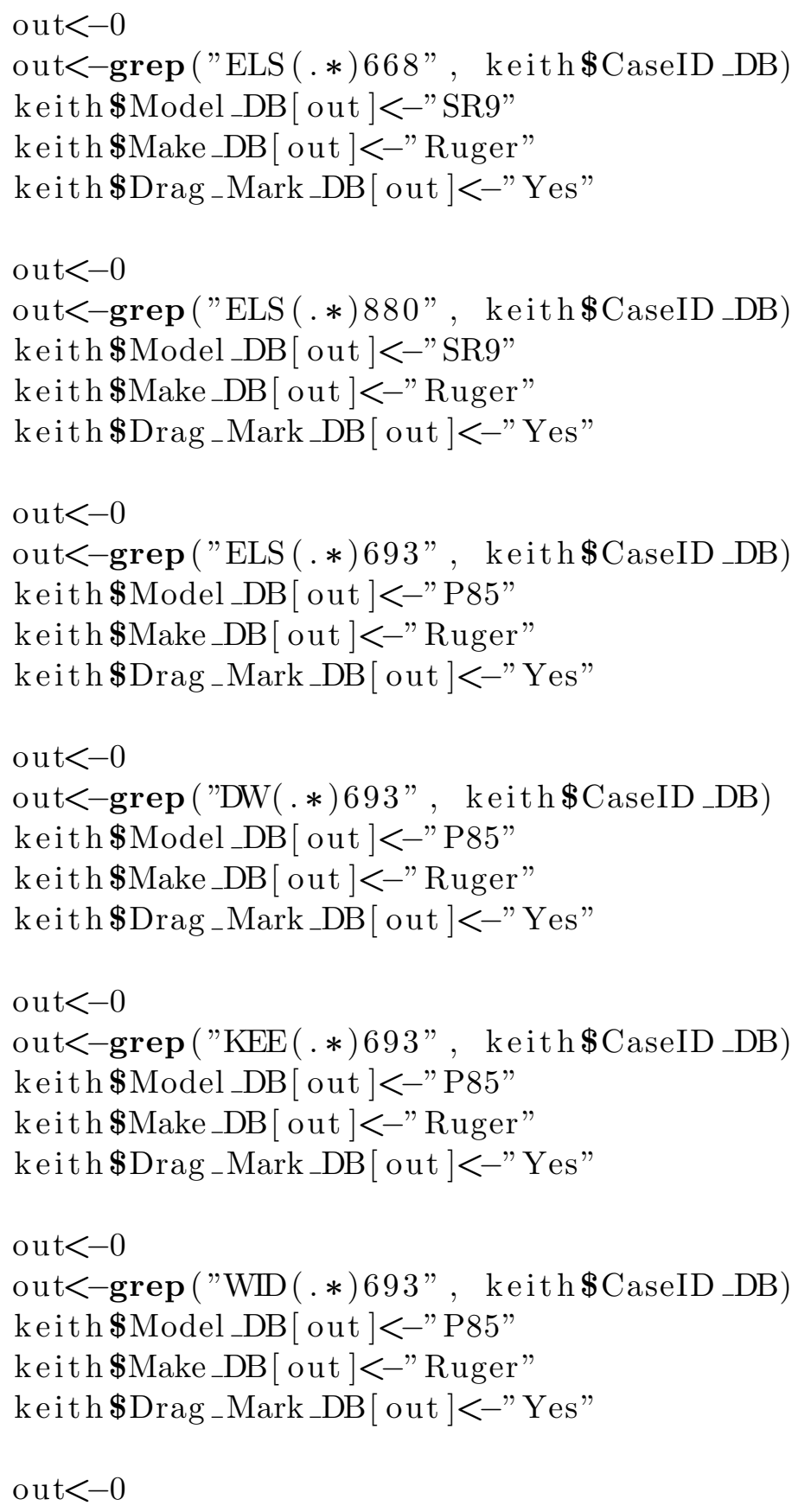




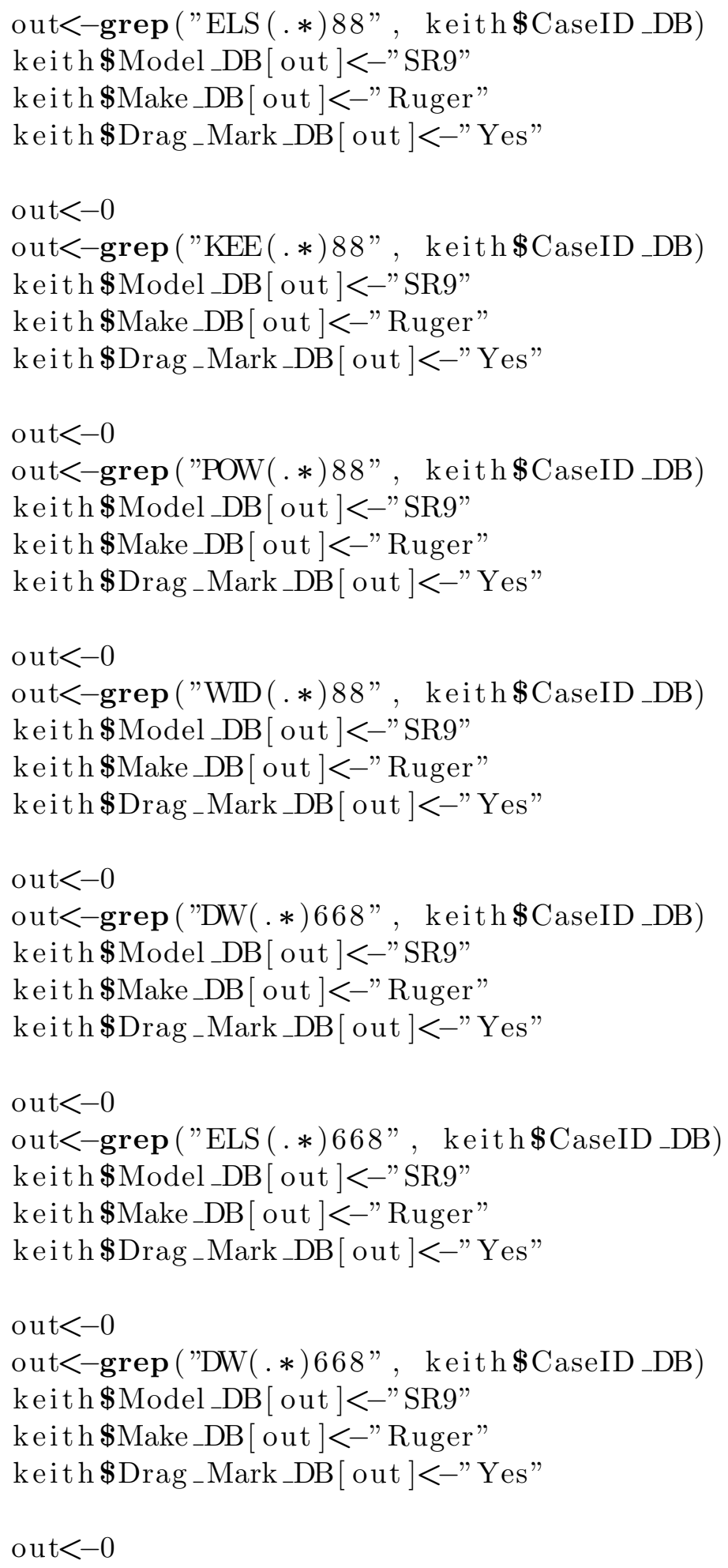




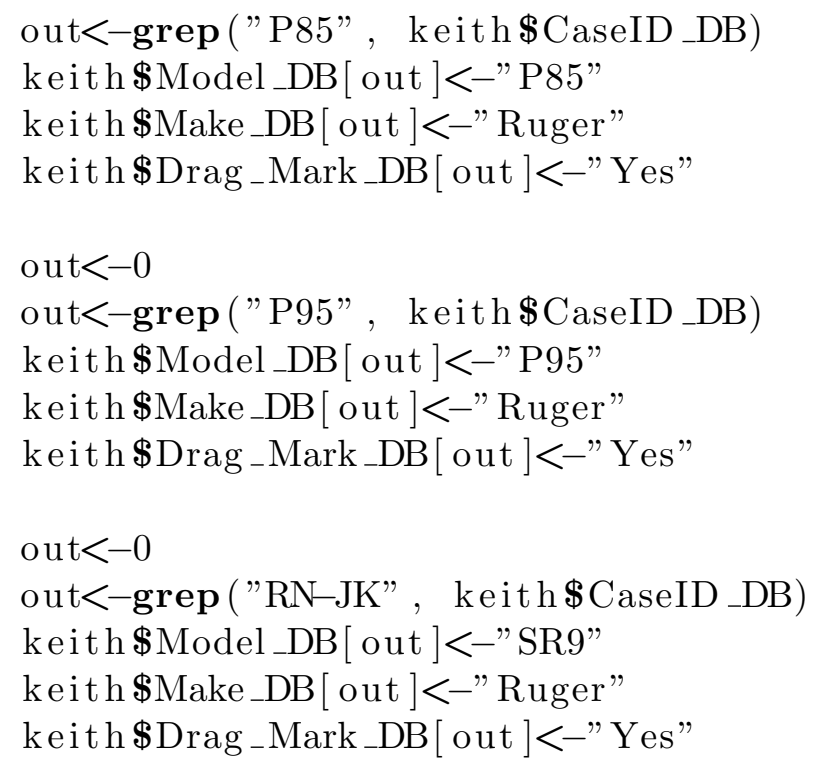

\subsection{Process Pressure Trace II $^{\mathrm{TM}}$ Shot Data}

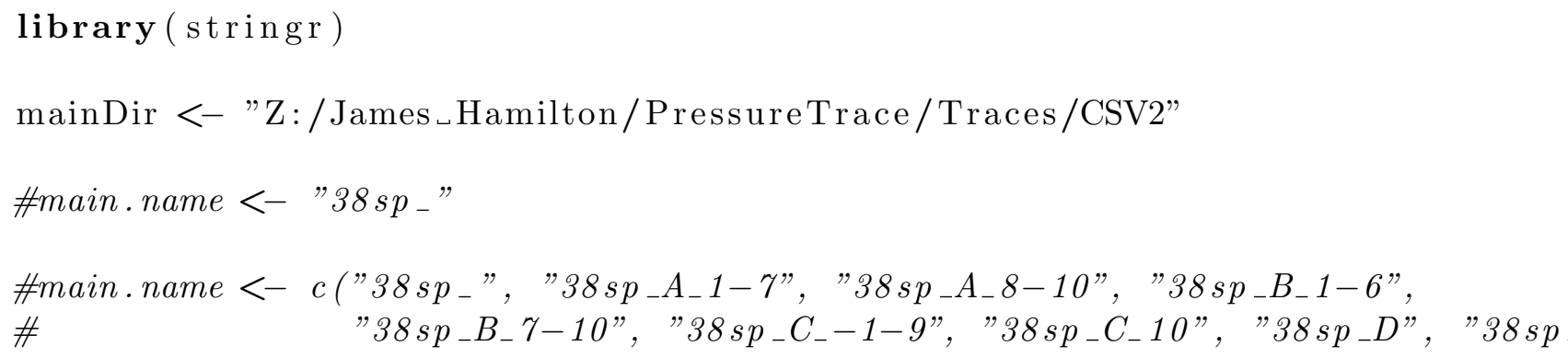




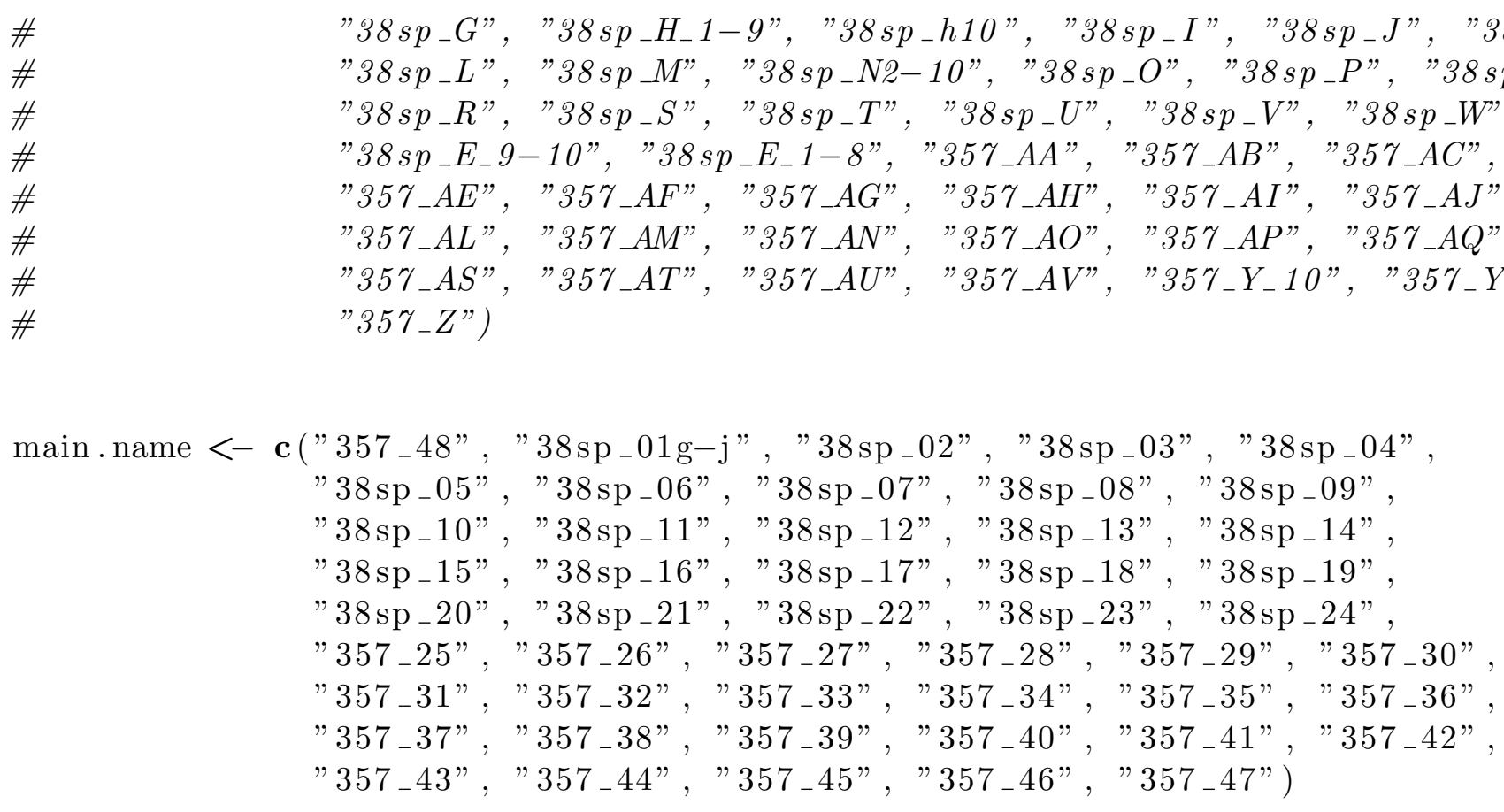

for ( $\mathrm{z}$ in main.name) \{

fname<-paste0 (mainDir, "/, , z, " .csv")

$\mathrm{m}=$ readLines (fname)

$\# m=$ readLines ("Z:/Firearms/Completed Files/9mm Text Files/AR98/AR98-BZ9

\#CaseID_Sample_str $<-$ substr(m[2], 25, 25+20-1)

strings $=\mathbf{c}\left("\right.$ Barrel ${ }_{\llcorner}=\mathrm{T} / \mathrm{C}_{\llcorner}$Contender $.357 "$,

$"$ Gage $\_$Factor $\_=\_2.1 "$,

"Bullet $\_$Diameter ${ }_{\lrcorner}=0.358 "$,

$"$ Barrel $\_$OD_= $=0.81 "$,

$"$ Barrel $\_$ID $\_=\_0.375 "$,

"Case $\_$Wall $\_$Thickness $\_=\_0.01 "$,

"Pressure ${ }_{\llcorner}$Correction ${ }={ }_{\llcorner} 0 "$,

"Barrel $\_$Length $=_{\llcorner} 12 "$,

"Case $\llcorner$ Length $\llcorner=\llcorner 1 "$,

"PSI $\left\llcorner\right.$ Average $=_{\sqcup}$ ",

"PSI $\lrcorner$ SD $=\llcorner "$, 
"PSI $\_$Prob $\_95 \% \_="$,

"PSI $\sqcup$ High ${ }_{\sqcup}="$,

"PSI $\llcorner$ Low $\llcorner="$ ",

"PSI $\_$Extreme Spread $_{\llcorner}=$S",

"Area $\_$Under $\_$Curve $\lrcorner$Average ${ }_{\lrcorner}="$,

"Area $\_$Under $\_$Curve $\_S D \_="$,

"Area $\_$Under $\_$Curve $\lrcorner$Prob $\_95 \%_{\lrcorner}={ }_{\lrcorner} "$,

"Area $\_$Under $\_$Curve $\_$High $\_=\_$",

"Area ${ }_{\lrcorner}$Under $\_$Curve $\_$Low $=_{\llcorner} "$,

"Area $\_$Under $\_$Curve $\_$Extreme $\_$Spread $=_{\triangleleft} "$,

"Rise $\_$Time $\_$Average $\_=\_"$,

"Rise $\_$Time $\_\mathrm{SD}={ }_{\llcorner} "$,

"Rise ${ }^{2}$ Time $\_$Prob $\_95 \% \_=$=",

"Rise $\_$Time $\_$High $\_=\_"$,

"Rise $\_$Time $\_$Low $\_=\_$",

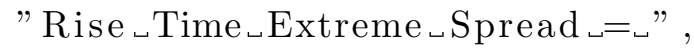

"Ambient $\_$Temperature ${ }_{\sqcup}="$ ",

"Barometric $\sqcup$ Pressure $==529.53$ ",

"Relative $\sqcup$ Humidity $\lrcorner=\sqcup 78$ ",

"Peak $\lrcorner$ Pressure $\lrcorner=\sqcup$,

"Peak Time $_{\llcorner}=\llcorner "$,

"Muzzle $\sqcup$ Velocity $\lrcorner={ } 0 "$,

"Bullet $\sqcup$ Weight $=_{\llcorner} 0 "$,

"Area $\_$Under ${ }_{\llcorner}$Curve ${ }_{\llcorner}={ }_{\llcorner} 1 "$,

"Rise $\sqcup$ Time $_{\sqcup}="$ ",

"Load $\_$Efficiency $==100 "$,

"End ¿Data",

"Chart_Notes",

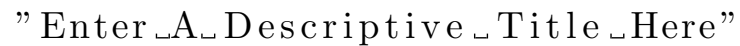

)

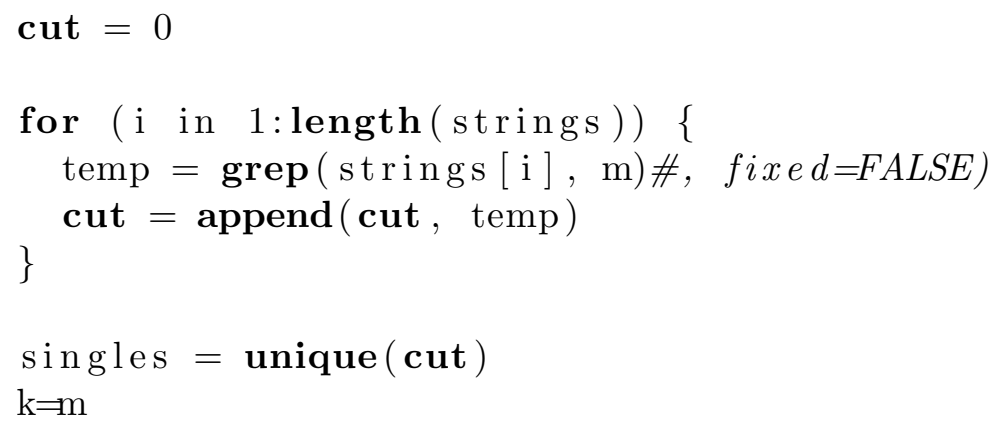




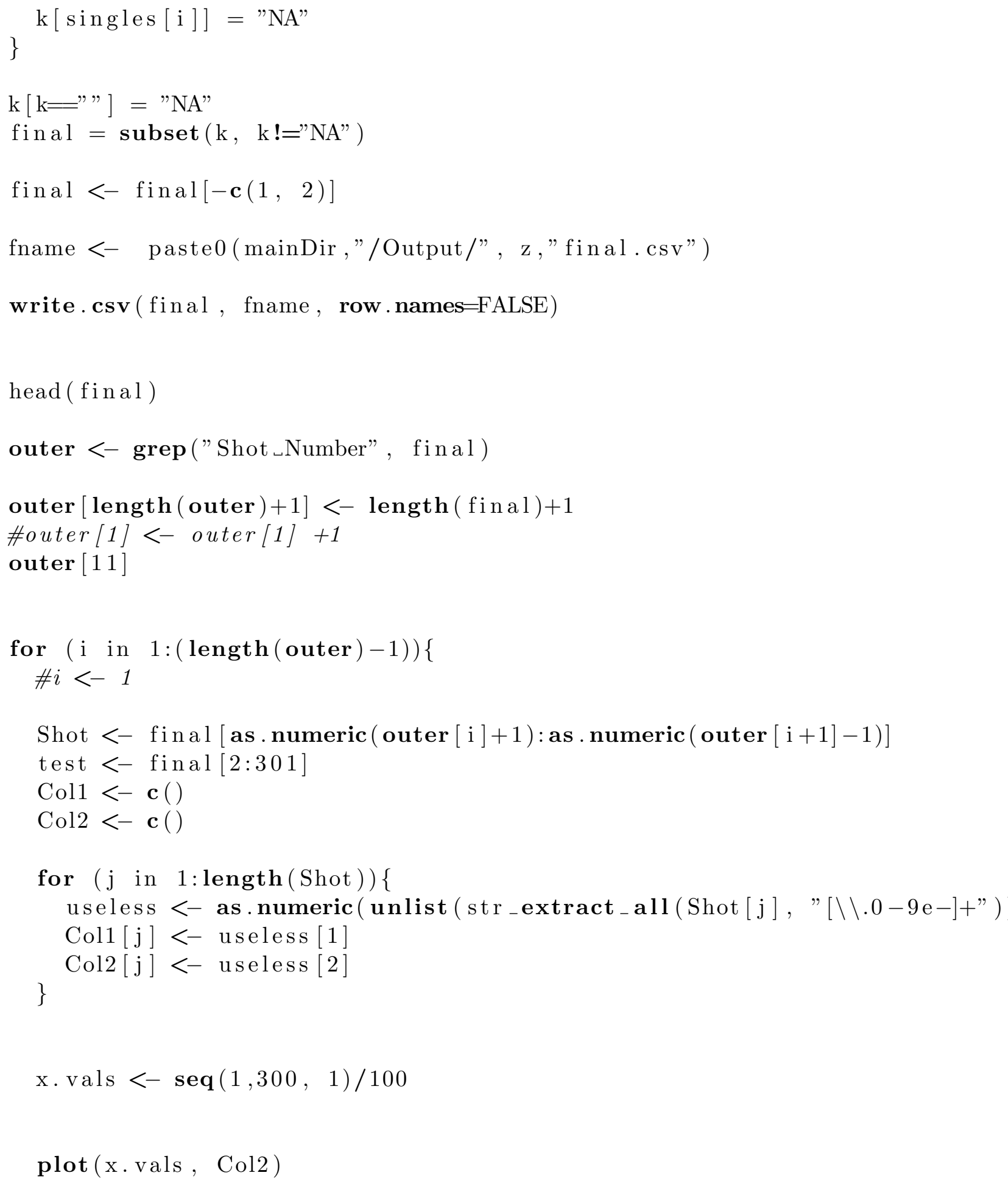




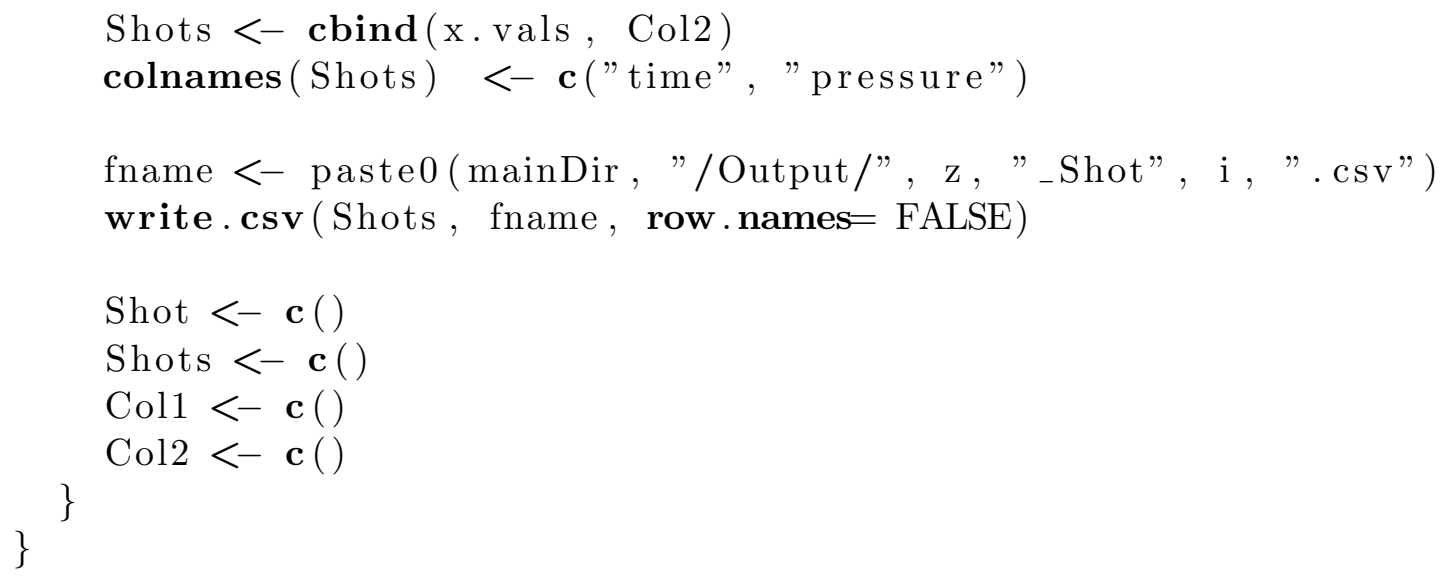

\subsection{Adjust Pressure Trace $\mathrm{II}^{\mathrm{TM}}$ Trace Data}

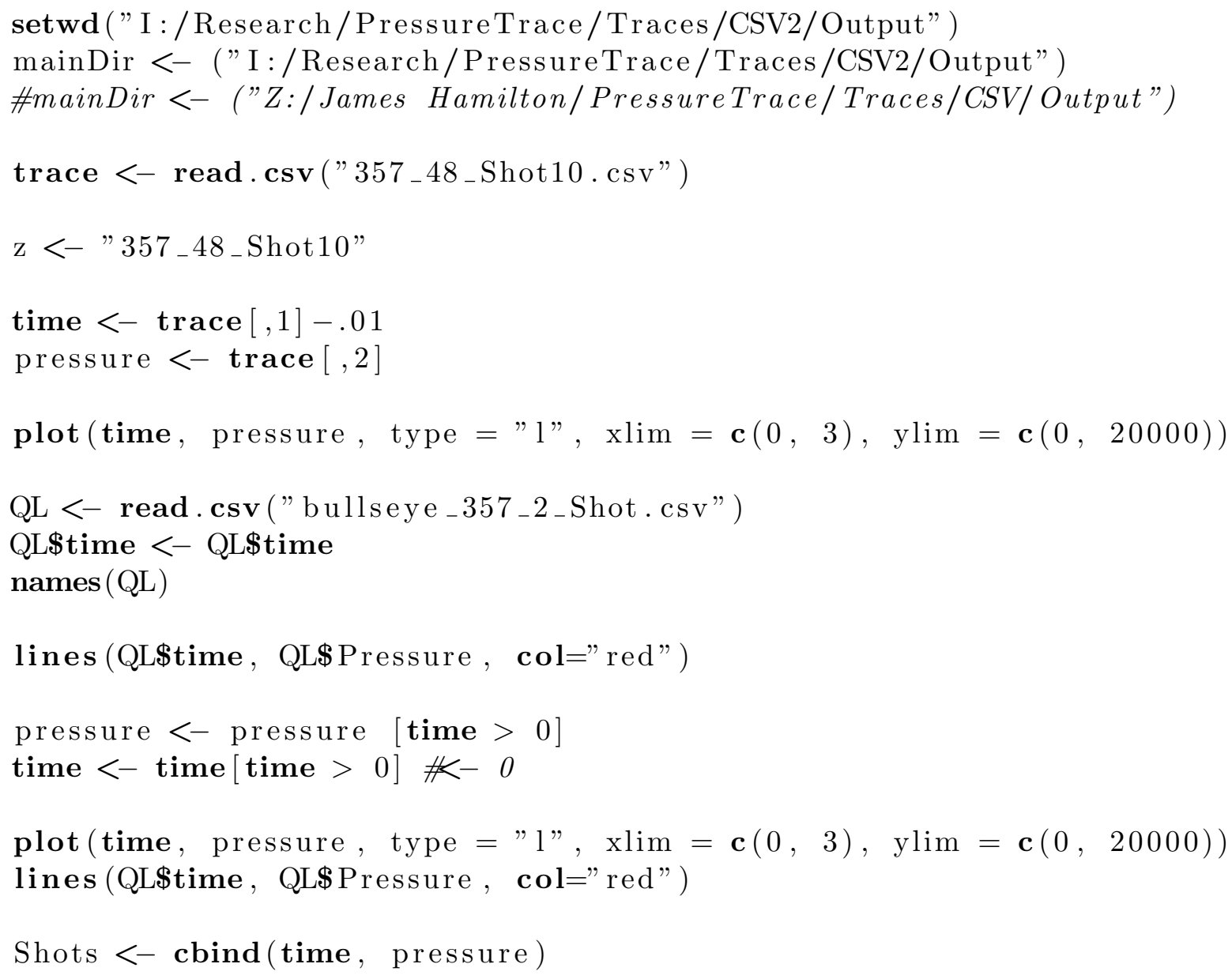


fname <- paste0 (mainDir, "/Adjusted/", z, "-Adjusted", ".csv") write.csv (Shots, fname, row.names= FALSE)

\subsection{Compile Pressure Trace II $^{\mathrm{TM}}$, Quickload ${ }^{\mathrm{TM}}$, and R Script Traces}

setwd("I : / Research / PressureTrace / Traces /CSV/Output/Adjusted") mainDir <- ("I : /Research/PressureTrace/Traces/CSV/Output/Adjusted") QL $<-\operatorname{read}$.csv ("bullseye_357_2_Shot.csv")

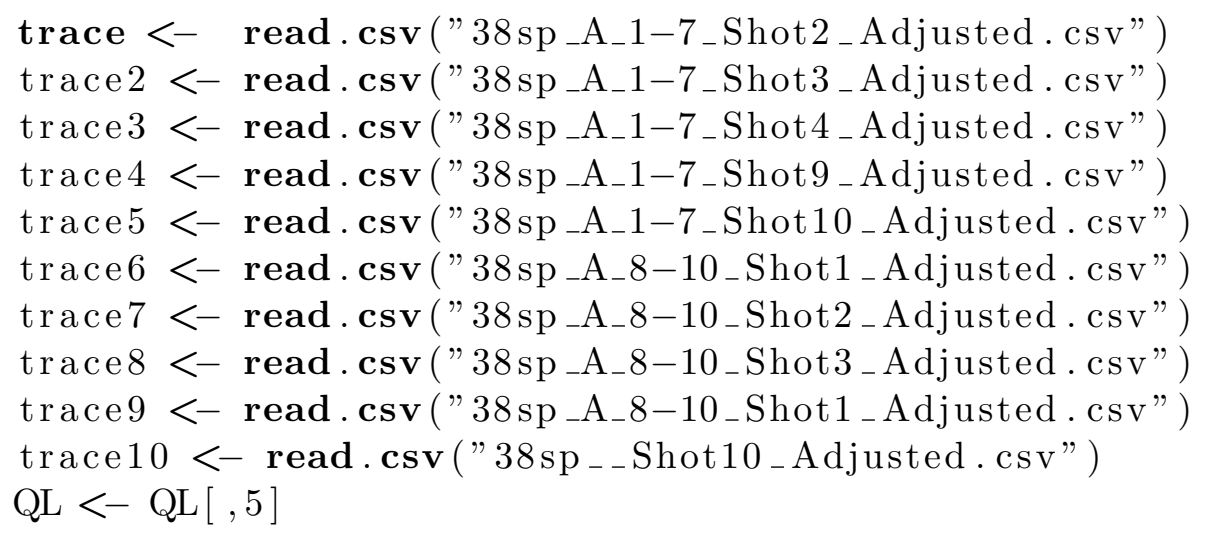




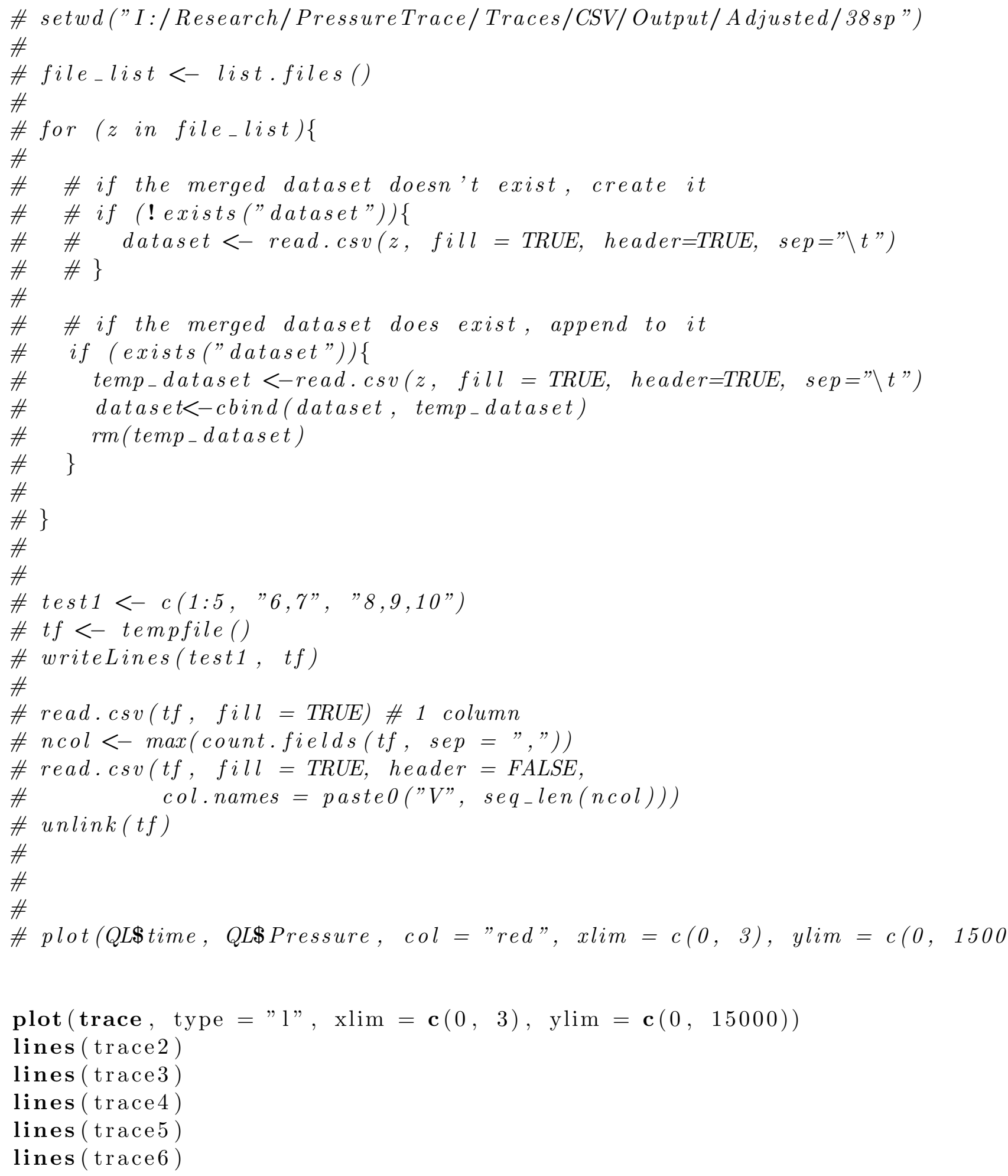




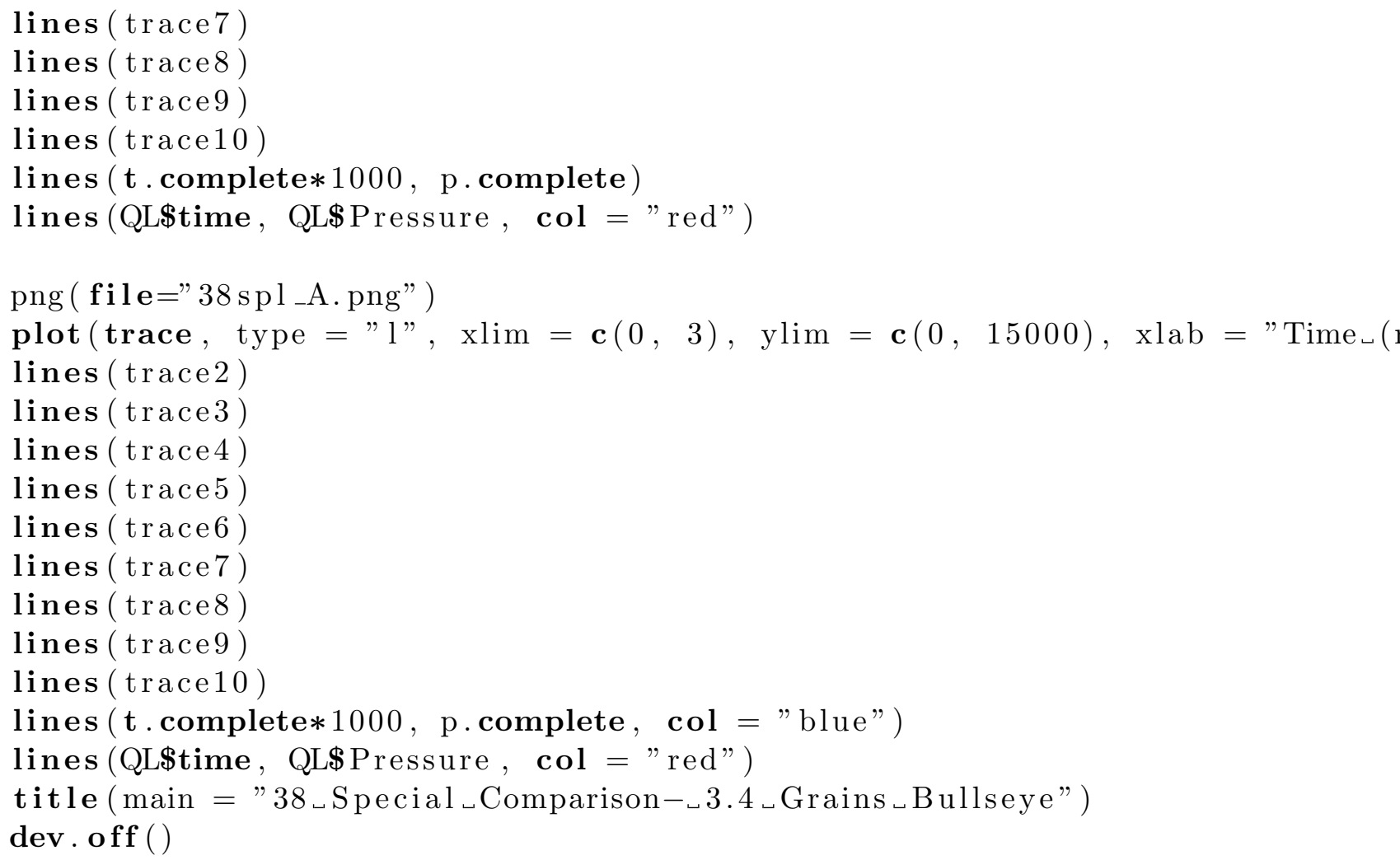

\subsection{Evaluation of IBIS ${ }^{\circledR}$ Data}

setwd ("I : / Research / IBIS /JH/PW_Excel_ Sheets")

mainDir <- " I : / Research/IBIS/JH/PW_Excel_Sheets"

library ( stringr)

library ( lattice)

memory. size $(\max =$ TRUE $)$

a $<-$ read.csv("newfile.csv")

a

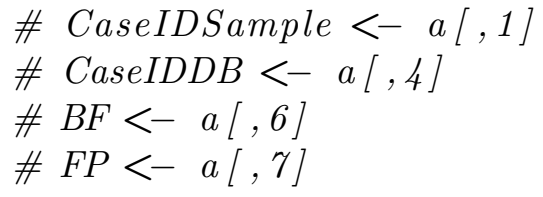




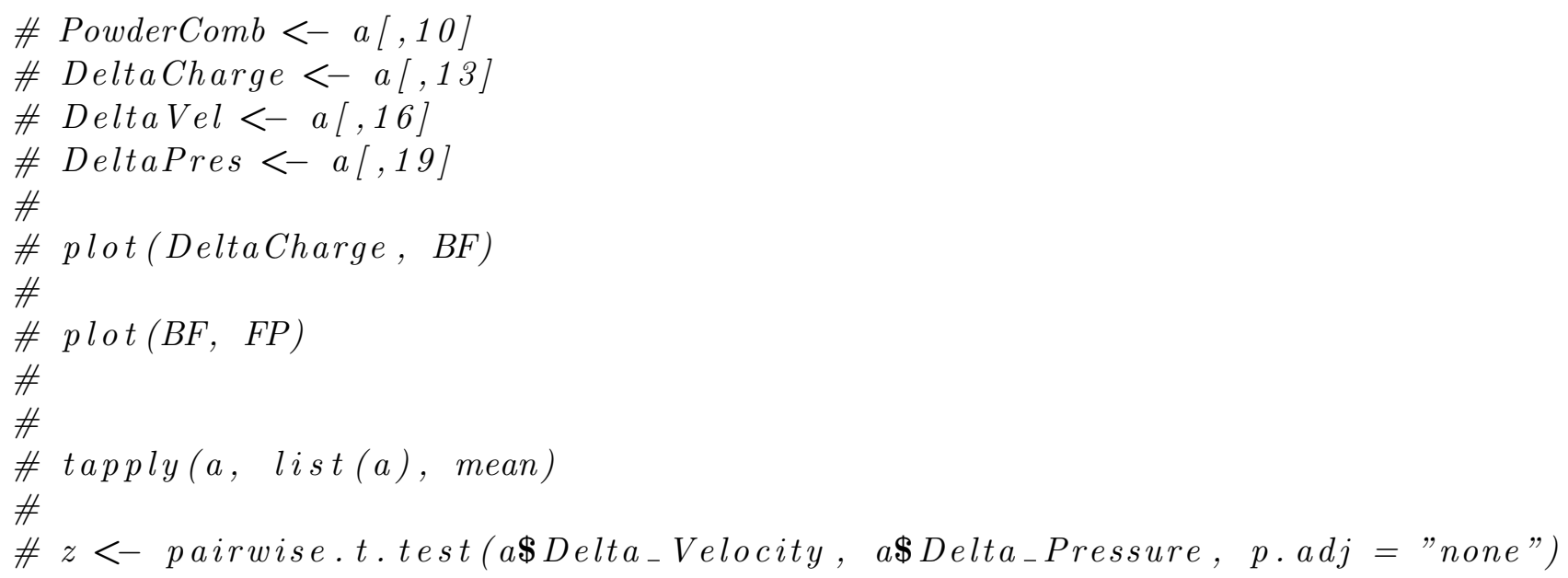


"CAT-UK-SRD-0511-0011",

"CAT-UK-SRD-0511-0015",

"CAT-UK-SRD-0511-0019",

"CAT-UK-SRD-0511-0023",

"CAT-UK-SRD-0511-0027",

"CAT-UK-SSB-0511-0001",

"CAT-UK-SSB-0511-0005",

"CAT-UK-SSB-0511-0009",

"CAT-UK-SSB-0511-0013",

"CAT-UK-SSB-0511-0017",

"CAT-UK-SSB-0511-0021",

"CAT-UK-SSB-0511-0025",

"CAT-UK-SSB-0511-0029",

"CAT-UK-SSD-0511-0003",

"CAT-UK-SSD-0511-0007",

"CAT-UK-SSD-0511-0011",

"CAT-UK-SSD-0511-0015",

"CAT-UK-SSD-0511-0019",

"CAT-UK-SSD-0511-0023",

"CAT-UK-SSD-0511-0027",

"CAT-UK-STB-0511-0001",

"CAT-UK-STB-0511-0005",

"CAT-UK-STB-0511-0009",

"CAT-UK-STB-0511-0013",

"CAT-UK-STB-0511-0017",

"CAT-UK-STB-0511-0021",

"CAT-UK-STB-0511-0025",

"CAT-UK-STB-0511-0029",

"CAT-UK-STD-0511-0003",

"CAT-UK-STD-0511-0007",

"CAT-UK-STD-0511-0011",

"CAT-UK-STD-0511-0015",

"CAT-UK-STD-0511-0019",

"CAT-UK-STD-0511-0023",

"CAT-UK-STD-0511-0027",

"CBT-UK-SFB-0511-0001",

"CBT-UK-SFB-0511-0005",

"CBT-UK-SFB-0511-0009",

"CBT-UK-SFB-0511-0013",

"CBT-UK-SFB-0511-0017",

"CBT-UK-SFB-0511-0021",

"CBT-UK-SFB-0511-0025",
"CAT-UK-SRD-0511-0012", "CAT-UK-SRD-0511-0016", "CAT-UK-SRD-0511-0020", "CAT-UK-SRD-0511-0024", "CAT-UK-SRD-0511-0028", "CAT-UK-SSB-0511-0002", "CAT-UK-SSB-0511-0006", "CAT-UK-SSB-0511-0010", "CAT-UK-SSB-0511-0014", "CAT-UK-SSB-0511-0018", "CAT-UK-SSB-0511-0022", "CAT-UK-SSB-0511-0026", "CAT-UK-SSB-0511-0030", "CAT-UK-SSD-0511-0004", "CAT-UK-SSD-0511-0008", "CAT-UK-SSD-0511-0012", "CAT-UK-SSD-0511-0016", "CAT-UK-SSD-0511-0020", "CAT-UK-SSD-0511-0024", "CAT-UK-SSD-0511-0028", "CAT-UK-STB-0511-0002", "CAT-UK-STB-0511-0006", "CAT-UK-STB-0511-0010", "CAT-UK-STB-0511-0014", "CAT-UK-STB-0511-0018", "CAT-UK-STB-0511-0022", "CAT-UK-STB-0511-0026", "CAT-UK-STB-0511-0030", "CAT-UK-STD-0511-0004", "CAT-UK-STD-0511-0008", "CAT-UK-STD-0511-0012", "CAT-UK-STD-0511-0016", "CAT-UK-STD-0511-0020", "CAT-UK-STD-0511-0024", "CAT-UK-STD-0511-0028", "CBT-UK-SFB-0511-0002", "CBT-UK-SFB-0511-0006", "CBT-UK-SFB-0511-0010", "CBT-UK-SFB-0511-0014", "CBT-UK-SFB-0511-0018", "CBT-UK-SFB-0511-0022", "CBT-UK-SFB-0511-0026",
"CAT-UK-SRD-05 "CAT-UK-SRD-05 "CAT-UK-SRD-05 "CAT-UK-SRD-05 "CAT-UK-SRD-05 "CAT-UK-SSB-05 "CAT-UK-SSB-05 "CAT-UK-SSB-05 "CAT-UK-SSB-05 "CAT-UK-SSB-0 5 "CAT-UK-SSB-05 "CAT-UK-SSB-05 "CAT-UK-SSD-05 "CAT-UK-SSD-05 "CAT-UK-SSD-05 "CAT-UK-SSD-05 "CAT-UK-SSD-05 "CAT-UK-SSD-05 "CAT-UK-SSD-05 "CAT-UK-SSD-05 "CAT-UK-STB-05 "CAT-UK-STB-05 "CAT-UK-STB-05 "CAT-UK-STB-05 "CAT-UK-STB-05 "CAT-UK-STB-05 "CAT-UK-STB-05 "CAT-UK-STD-05 "CAT-UK-STD-05 "CAT-UK-STD-05 "CAT-UK-STD-05 "CAT-UK-STD-05 "CAT-UK-STD-05 "CAT-UK-STD-05 "CAT-UK-STD-05 "CBT-UK-SFB-05 "CBT-UK-SFB-05 "CBT-UK-SFB-05 "CBT-UK-SFB-05 "CBT-UK-SFB-05 "CBT-UK-SFB-05 "CBT-UK-SFB-05 
"CBT-UK-SFB-0511-0029",

"CBT-UK-SFD-0511-0003",

"CBT-UK-SFD-0511-0007",

"CBT-UK-SFD-0511-0011",

"CBT-UK-SFD-0511-0015",

"CBT-UK-SFD-0511-0019",

"CBT-UK-SFD-0511-0023",

"CBT-UK-SFD-0511-0027",

"CBT-UK-SRB-0511-0001",

"CBT-UK-SRB-0511-0005",

"CBT-UK-SRB-0511-0009",

"CBT-UK-SRB-0511-0013",

"CBT-UK-SRB-0511-0017",

"CBT-UK-SRB-0511-0021",

"CBT-UK-SRB-0511-0025",

"CBT-UK-SRB-0511-0029",

"CBT-UK-SRD-0511-0003",

"CBT-UK-SRD-0511-0007",

"CBT-UK-SRD-0511-0011",

"CBT-UK-SRD-0511-0015" ,

"CBT-UK-SRD-0511-0019",

"CBT-UK-SRD-0511-0023",

"CBT-UK-SRD-0511-0027",

"CBT-UK-SSB-0511-0001",

"CBT-UK-SSB-0511-0005",

"CBT-UK-SSB-0511-0009",

"CBT-UK-SSB-0511-0013",

"CBT-UK-SSB-0511-0017",

"CBT-UK-SSB-0511-0021",

"CBT-UK-SSB-0511-0025",

"CBT-UK-SSB-0511-0029",

"CBT-UK-SSD-0511-0003",

"CBT-UK-SSD-0511-0007",

"CBT-UK-SSD-0511-0011",

"CBT-UK-SSD-0511-0015",

"CBT-UK-SSD-0511-0019",

"CBT-UK-SSD-0511-0023",

"CBT-UK-SSD-0511-0027",

"CBT-UK-STB-0511-0001",

"CBT-UK-STB-0511-0005",

"CBT-UK-STB-0511-0009",

"CBT-UK-STB-0511-0013",
"CBT-UK-SFB-0511-0030",

"CBT-UK-SFD-0511-0004",

"CBT-UK-SFD-0511-0008",

"CBT-UK-SFD-0511-0012",

"CBT-UK-SFD-0511-0016",

"CBT-UK-SFD-0511-0020",

"CBT-UK-SFD-0511-0024",

"CBT-UK-SFD-0511-0028",

"CBT-UK-SRB-0511-0002",

"CBT-UK-SRB-0511-0006",

"CBT-UK-SRB-0511-0010",

"CBT-UK-SRB-0511-0014",

"CBT-UK-SRB-0511-0018",

"CBT-UK-SRB-0511-0022",

"CBT-UK-SRB-0511-0026",

"CBT-UK-SRB-0511-0030",

"CBT-UK-SRD-0511-0004",

"CBT-UK-SRD-0511-0008",

"CBT-UK-SRD-0511-0012",

"CBT-UK-SRD-0511-0016",

"CBT-UK-SRD-0511-0020",

"CBT-UK-SRD-0511-0024",

"CBT-UK-SRD-0511-0028",

"CBT-UK-SSB-0511-0002",

"CBT-UK-SSB-0511-0006",

"CBT-UK-SSB-0511-0010",

"CBT-UK-SSB-0511-0014",

"CBT-UK-SSB-0511-0018",

"CBT-UK-SSB-0511-0022",

"CBT-UK-SSB-0511-0026",

"CBT-UK-SSB-0511-0030",

"CBT-UK-SSD-0511-0004",

"CBT-UK-SSD-0511-0008",

"CBT-UK-SSD-0511-0012",

"CBT-UK-SSD-0511-0016",

"CBT-UK-SSD-0511-0020",

"CBT-UK-SSD-0511-0024",

"CBT-UK-SSD-0511-0028",

"CBT-UK-STB-0511-0002",

"CBT-UK-STB-0511-0006",

"CBT-UK-STB-0511-0010",

"CBT-UK-STB-0511-0014",
"CBT-UK-SFD-05

"CBT-UK-SFD-05

"CBT-UK-SFD-05

"CBT-UK-SFD-0 5

"CBT-UK-SFD-05

"CBT-UK-SFD-05

"CBT-UK-SFD-05

"CBT-UK-SFD-05

"CBT-UK-SRB-05

"CBT-UK-SRB-05

"CBT-UK-SRB-05

"CBT-UK-SRB-05

"CBT-UK-SRB-05

"CBT-UK-SRB-05

"CBT-UK-SRB-0 5

"CBT-UK-SRD-05

"CBT-UK-SRD-05

"CBT-UK-SRD-05

"CBT-UK-SRD-05

"CBT-UK-SRD-05

"CBT-UK-SRD-0 5

"CBT-UK-SRD-05

"CBT-UK-SRD-0 5

"CBT-UK-SSB-0 5

"CBT-UK-SSB-05

"CBT-UK-SSB-05

"CBT-UK-SSB-05

"CBT-UK-SSB-05

"CBT-UK-SSB-05

"CBT-UK-SSB-0 05

"CBT-UK-SSD-05

"CBT-UK-SSD-05

"CBT-UK-SSD-05

"CBT-UK-SSD-05

"CBT-UK-SSD-05

"CBT-UK-SSD-05

"CBT-UK-SSD-05

"CBT-UK-SSD-05

"CBT-UK-STB-05

"CBT-UK-STB-05

"CBT-UK-STB-05

"CBT-UK-STB-05 
"CBT-UK-STB-0511-0017",

"CBT-UK-STB-0511-0021",

"CBT-UK-STB-0511-0025",

"CBT-UK-STB-0511-0029",

"CBT-UK-STD-0511-0003",

"CBT-UK-STD-0511-0007",

"CBT-UK-STD-0511-0011",

"CBT-UK-STD-0511-0015",

"CBT-UK-STD-0511-0019",

"CBT-UK-STD-0511-0023",

"CBT-UK-STD-0511-0027",

"CCT-UK-SFB-0614-0001",

"CCT-UK-SFB-0614-0005",

"CCT-UK-SFB-0614-0009", ,

"CCT-UK-SFB-0614-0013",

"CCT-UK-SFB-0614-0017",

"CCT-UK-SFB-0614-0021",

"CCT-UK-SFB-0614-0025",

"CCT-UK-SFB-0614-0029",

"CCT-UK-SFD-0614-0003",

"CCT-UK-SFD-0614-0007",

"CCT-UK-SFD-0614-0011",

"CCT-UK-SFD-0614-0015",

"CCT-UK-SFD-0614-0019",

"CCT-UK-SFD-0614-0023",

"CCT-UK-SFD-0614-0027",

"CCT-UK-SRB-0614-0001",

"CCT-UK-SRB-0614-0005",

"CCT-UK-SRB-0614-0009",

"CCT-UK-SRB-0614-0013",

"CCT-UK-SRB-0614-0017",

"CCT-UK-SRB-0614-0021",

"CCT-UK-SRB-0614-0025",

"CCT-UK-SRB-0614-0029",

"CCT-UK-SRD-0614-0003",

"CCT-UK-SRD-0614-0007",

"CCT-UK-SRD-0614-0011",

"CCT-UK-SRD-0614-0015",

"CCT-UK-SRD-0614-0019",

"CCT-UK-SRD-0614-0023",

"CCT-UK-SRD-0614-0027",

"CCT-UK-SSB-0614-0001",
"CBT-UK-STB-0511-0018",

"CBT-UK-STB-0511-0022",

"CBT-UK-STB-0511-0026",

"CBT-UK-STB-0511-0030",

"CBT-UK-STD-0511-0004",

"CBT-UK-STD-0511-0008",

"CBT-UK-STD-0511-0012",

"CBT-UK-STD-0511-0016",

"CBT-UK-STD-0511-0020",

"CBT-UK-STD-0511-0024",

"CBT-UK-STD-0511-0028",

"CCT-UK-SFB-0614-0002",

"CCT-UK-SFB-0614-0006",

"CCT-UK-SFB-0614-0010",

"CCT-UK-SFB-0614-0014",

"CCT-UK-SFB-0614-0018",

"CCT-UK-SFB-0614-0022" ,

"CCT-UK-SFB-0614-0026",

"CCT-UK-SFB-0614-0030",

"CCT-UK-SFD-0614-0004",

"CCT-UK-SFD-0614-0008",

"CCT-UK-SFD-0614-0012",

"CCT-UK-SFD-0614-0016",

"CCT-UK-SFD-0614-0020",

"CCT-UK-SFD-0614-0024",

"CCT-UK-SFD-0614-0028",

"CCT-UK-SRB-0614-0002" ,

"CCT-UK-SRB-0614-0006",

"CCT-UK-SRB-0614-0010",

"CCT-UK-SRB-0614-0014",

"CCT-UK-SRB-0614-0018",

"CCT-UK-SRB-0614-0022",

"CCT-UK-SRB-0614-0026",

"CCT-UK-SRB-0614-0030",

"CCT-UK-SRD-0614-0004",

"CCT-UK-SRD-0614-0008",

"CCT-UK-SRD-0614-0012",

"CCT-UK-SRD-0614-0016",

"CCT-UK-SRD-0614-0020" ,

"CCT-UK-SRD-0614-0024",

"CCT-UK-SRD-0614-0028",

"CCT-UK-SSB-0614-0002",
"CBT-UK-STB-05

"CBT-UK-STB-05

"CBT-UK-STB-05

"CBT-UK-STD-05

"CBT-UK-STD-05

"CBT-UK-STD-05

"CBT-UK-STD-0 5

"CBT-UK-STD-05

"CBT-UK-STD-05

"CBT-UK-STD-05

"CBT-UK-STD-05

"CCT-UK-SFB-06

"CCT-UK-SFB-06

"CCT-UK-SFB-06

"CCT-UK-SFB-06

"CCT-UK-SFB-06

"CCT-UK-SFB-06

"CCT-UK-SFB-06

"CCT-UK-SFD-06

"CCT-UK-SFD-06

"CCT-UK-SFD-06

"CCT-UK-SFD-06

"CCT-UK-SFD-06

"CCT-UK-SFD-06

"CCT-UK-SFD-06

"CCT-UK-SFD-06

"CCT-UK-SRB-06

"CCT-UK-SRB-06

"CCT-UK-SRB-06

"CCT-UK-SRB-06

"CCT-UK-SRB-06

"CCT-UK-SRB-06

"CCT-UK-SRB-06

"CCT-UK-SRD-06

"CCT-UK-SRD-06

"CCT-UK-SRD-06

"CCT-UK-SRD-06

"CCT-UK-SRD-06

"CCT-UK-SRD-06

"CCT-UK-SRD-06

"CCT-UK-SRD-06

"CCT-UK-SSB-06 
"CCT-UK-SSB-0614-0005" ,

"CCT-UK-SSB-0614-0009",

"CCT-UK-SSB-0614-0013",

"CCT-UK-SSB-0614-0017",

"CCT-UK-SSB-0614-0021",

"CCT-UK-SSB-0614-0025",

"CCT-UK-SSB-0614-0029",

"CCT-UK-SSD-0614-0003",

"CCT-UK-SSD-0614-0007",

"CCT-UK-SSD-0614-0011",

"CCT-UK-SSD-0614-0015",

"CCT-UK-SSD-0614-0019",

"CCT-UK-SSD-0614-0023",

"CCT-UK-SSD-0614-0027",

"CCT-UK-STB-0614-0001",

"CCT-UK-STB-0614-0005",

"CCT-UK-STB-0614-0009",

"CCT-UK-STB-0614-0013",

"CCT-UK-STB-0614-0017",

"CCT-UK-STB-0614-0021",

"CCT-UK-STB-0614-0025",

"CCT-UK-STB-0614-0029",

"CCT-UK-STD-0614-0003",

"CCT-UK-STD-0614-0007",

"CCT-UK-STD-0614-0011",

"CCT-UK-STD-0614-0015",

"CCT-UK-STD-0614-0019",

"CCT-UK-STD-0614-0023",

"CCT-UK-STD-0614-0027",

"CDT-UK-SFB-0614-0001",

"CDT-UK-SFB-0614-0005",

"CDT-UK-SFB-0614-0009",

"CDT-UK-SFB-0614-0013",

"CDT-UK-SFB-0614-0017",

"CDT-UK-SFB-0614-0021",

"CDT-UK-SFB-0614-0025",

"CDT-UK-SFB-0614-0029",

"CDT-UK-SFD-0614-0003",

"CDT-UK-SFD-0614-0007",

"CDT-UK-SFD-0614-0011",

"CDT-UK-SFD-0614-0015",

"CDT-UK-SFD-0614-0019",
"CCT-UK-SSB-0614-0006" , "CCT-UK-SSB-0614-0010", "CCT-UK-SSB-0614-0014", "CCT-UK-SSB-0614-0018", "CCT-UK-SSB-0614-0022", "CCT-UK-SSB-0614-0026", "CCT-UK-SSB-0614-0030", "CCT-UK-SSD-0614-0004", "CCT-UK-SSD-0614-0008", "CCT-UK-SSD-0614-0012", "CCT-UK-SSD-0614-0016", "CCT-UK-SSD-0614-0020", "CCT-UK-SSD-0614-0024", "CCT-UK-SSD-0614-0028", "CCT-UK-STB-0614-0002", "CCT-UK-STB-0614-0006", "CCT-UK-STB-0614-0010" , "CCT-UK-STB-0614-0014", "CCT-UK-STB-0614-0018", "CCT-UK-STB-0614-0022", "CCT-UK-STB-0614-0026", "CCT-UK-STB-0614-0030" , "CCT-UK-STD-0614-0004", "CCT-UK-STD-0614-0008", "CCT-UK-STD-0614-0012", "CCT-UK-STD-0614-0016", "CCT-UK-STD-0614-0020", "CCT-UK-STD-0614-0024", "CCT-UK-STD-0614-0028", "CDT-UK-SFB-0614-0002", "CDT-UK-SFB-0614-0006", "CDT-UK-SFB-0614-0010", "CDT-UK-SFB-0614-0014", "CDT-UK-SFB-0614-0018", "CDT-UK-SFB-0614-0022", "CDT-UK-SFB-0614-0026", "CDT-UK-SFB-0614-0030", "CDT-UK-SFD-0614-0004", "CDT-UK-SFD-0614-0008", "CDT-UK-SFD-0614-0012", "CDT-UK-SFD-0614-0016", "CDT-UK-SFD-0614-0020",
"CCT-UK-SSB-06 "CCT-UK-SSB-06 "CCT-UK-SSB-06 "CCT-UK-SSB-06 "CCT-UK-SSB-06 "CCT-UK-SSB-06 "CCT-UK-SSD-06 "CCT-UK-SSD-06 "CCT-UK-SSD-06 "CCT-UK-SSD-06 "CCT-UK-SSD-06 "CCT-UK-SSD-06 "CCT-UK-SSD-06 "CCT-UK-SSD-06 "CCT-UK-STB-06 "CCT-UK-STB-06 "CCT-UK-STB-06 "CCT-UK-STB-06 "CCT-UK-STB-06 "CCT-UK-STB-06 "CCT-UK-STB-06 "CCT-UK-STD-06 "CCT-UK-STD-06 "CCT-UK-STD-06 "CCT-UK-STD-06 "CCT-UK-STD-06 "CCT-UK-STD-06 "CCT-UK-STD-06 "CCT-UK-STD-06 "CDT-UK-SFB-06 "CDT-UK-SFB-06 "CDT-UK-SFB-06 "CDT-UK-SFB-06 "CDT-UK-SFB-06 "CDT-UK-SFB-06 "CDT-UK-SFB-06 "CDT-UK-SFD-06 "CDT-UK-SFD-06 "CDT-UK-SFD-06 "CDT-UK-SFD-06 "CDT-UK-SFD-06 "CDT-UK-SFD-06 
"CDT-UK-SFD-0614-0023",

"CDT-UK-SFD-0614-0027",

"CDT-UK-SRB-0614-0001",

"CDT-UK-SRB-0614-0005",

"CDT-UK-SRB-0614-0009",

"CDT-UK-SRB-0614-0013",

"CDT-UK-SRB-0614-0017",

"CDT-UK-SRB-0614-0021",

"CDT-UK-SRB-0614-0025",

"CDT-UK-SRB-0614-0029",

"CDT-UK-SRD-0614-0003",

"CDT-UK-SRD-0614-0007",

"CDT-UK-SRD-0614-0011",

"CDT-UK-SRD-0614-0015",

"CDT-UK-SRD-0614-0019",

"CDT-UK-SRD-0614-0023",

"CDT-UK-SRD-0614-0027",

"CDT-UK-SSB-0614-0001",

"CDT-UK-SSB-0614-0005",

"CDT-UK-SSB-0614-0009",

"CDT-UK-SSB-0614-0013",

"CDT-UK-SSB-0614-0017",

"CDT-UK-SSB-0614-0021",

"CDT-UK-SSB-0614-0025",

"CDT-UK-SSB-0614-0029",

"CDT-UK-SSD-0614-0003",

"CDT-UK-SSD-0614-0007",

"CDT-UK-SSD-0614-0011",

"CDT-UK-SSD-0614-0015",

"CDT-UK-SSD-0614-0019",

"CDT-UK-SSD-0614-0023",

"CDT-UK-SSD-0614-0027",

"CDT-UK-STB-0614-0001",

"CDT-UK-STB-0614-0005",

"CDT-UK-STB-0614-0009",

"CDT-UK-STB-0614-0013",

"CDT-UK-STB-0614-0017",

"CDT-UK-STB-0614-0021",

"CDT-UK-STB-0614-0025",

"CDT-UK-STB-0614-0029",

"CDT-UK-STD-0614-0003",

"CDT-UK-STD-0614-0007",
"CDT-UK-SFD-0614-0024", "CDT-UK-SFD-0614-0028", "CDT-UK-SRB-0614-0002", "CDT-UK-SRB-0614-0006", "CDT-UK-SRB-0614-0010", "CDT-UK-SRB-0614-0014", "CDT-UK-SRB-0614-0018", "CDT-UK-SRB-0614-0022", "CDT-UK-SRB-0614-0026", "CDT-UK-SRB-0614-0030", "CDT-UK-SRD-0614-0004", "CDT-UK-SRD-0614-0008", "CDT-UK-SRD-0614-0012", "CDT-UK-SRD-0614-0016", "CDT-UK-SRD-0614-0020", "CDT-UK-SRD-0614-0024", "CDT-UK-SRD-0614-0028", "CDT-UK-SSB-0614-0002", "CDT-UK-SSB-0614-0006", "CDT-UK-SSB-0614-0010", "CDT-UK-SSB-0614-0014", "CDT-UK-SSB-0614-0018", "CDT-UK-SSB-0614-0022", "CDT-UK-SSB-0614-0026", "CDT-UK-SSB-0614-0030", "CDT-UK-SSD-0614-0004", "CDT-UK-SSD-0614-0008", "CDT-UK-SSD-0614-0012", "CDT-UK-SSD-0614-0016", "CDT-UK-SSD-0614-0020", "CDT-UK-SSD-0614-0024", "CDT-UK-SSD-0614-0028", "CDT-UK-STB-0614-0002", "CDT-UK-STB-0614-0006", "CDT-UK-STB-0614-0010", "CDT-UK-STB-0614-0014", "CDT-UK-STB-0614-0018", "CDT-UK-STB-0614-0022", "CDT-UK-STB-0614-0026", "CDT-UK-STB-0614-0030", "CDT-UK-STD-0614-0004", "CDT-UK-STD-0614-0008",
"CDT-UK-SFD-06 "CDT-UK-SFD-06 "CDT-UK-SRB-06 "CDT-UK-SRB-06 "CDT-UK-SRB-06 "CDT-UK-SRB-06 "CDT-UK-SRB-06 "CDT-UK-SRB-06 "CDT-UK-SRB-06 "CDT-UK-SRD-06 "CDT-UK-SRD-06 "CDT-UK-SRD-06 "CDT-UK-SRD-06 "CDT-UK-SRD-06 "CDT-UK-SRD-06 "CDT-UK-SRD-06 "CDT-UK-SRD-06 "CDT-UK-SSB-06 "CDT-UK-SSB-06 "CDT-UK-SSB-06 "CDT-UK-SSB-06 "CDT-UK-SSB-06 "CDT-UK-SSB-06 "CDT-UK-SSB-06 "CDT-UK-SSD-06 "CDT-UK-SSD-06 "CDT-UK-SSD-06 "CDT-UK-SSD-06 "CDT-UK-SSD-06 "CDT-UK-SSD-06 "CDT-UK-SSD-06 "CDT-UK-SSD-06 "CDT-UK-STB-06 "CDT-UK-STB-06 "CDT-UK-STB-06 "CDT-UK-STB-06 "CDT-UK-STB-06 "CDT-UK-STB-06 "CDT-UK-STB-06 "CDT-UK-STD-06 "CDT-UK-STD-06 "CDT-UK-STD-06 


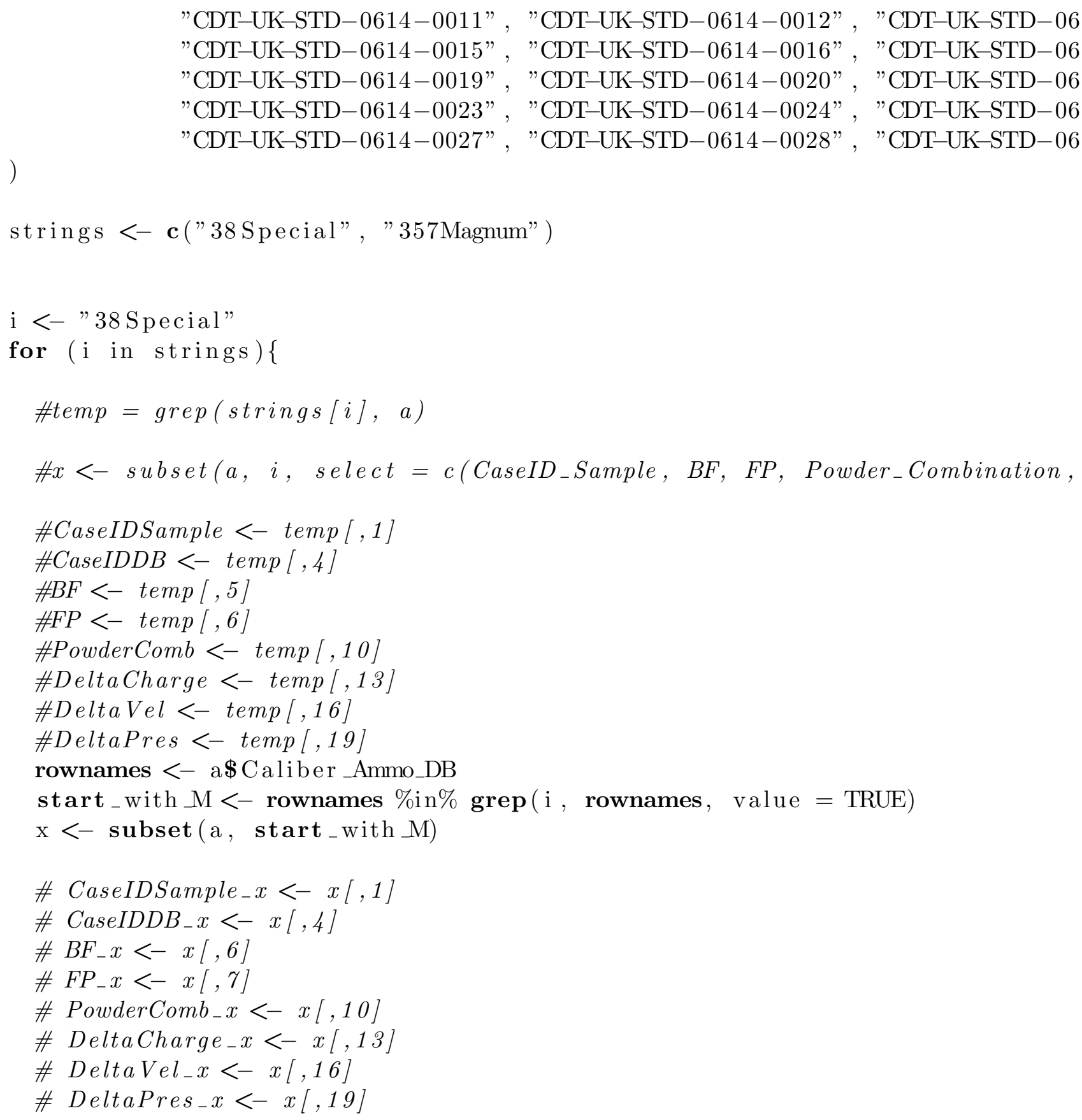




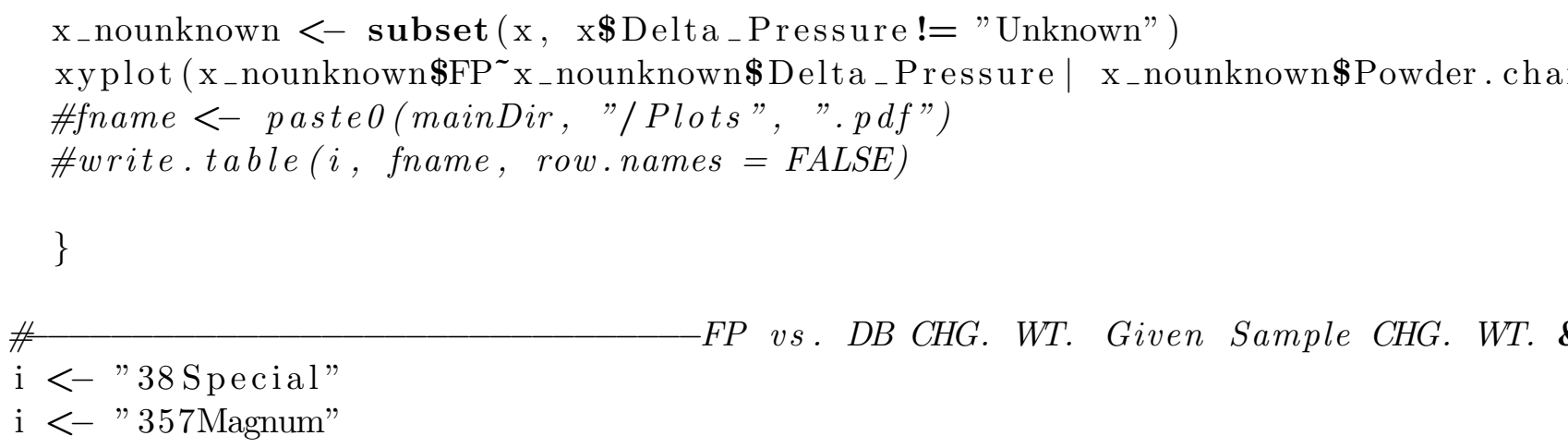

rownames <- a\$Caliber_Ammo_Sample start_with $\_<-$rownames $\%$ in $\% \operatorname{grep}(i$, rownames, value $=$ TRUE) $\mathrm{x}<-\operatorname{subset}\left(\mathrm{a}, \quad\right.$ start $\_$with $\_$)

Powder . charge_DB $<-$ paste0 (x\$Powder_Sample, "-" , x\$Powder_Charge_Sample) $\mathrm{x}<-\operatorname{cbind}\left(\mathrm{x}\right.$, Powder. charge $\left.\_\mathrm{DB}\right)$

$\# p l o t\left(B F_{-} x, F P_{-} x, \operatorname{main}=i, \quad x l a b=" B F "\right.$, ylab $=" F P "$, col $=$ ifelse $(x[, 20$

$\mathrm{x}_{-}$nounknown $<-\operatorname{subset}(\mathrm{x}, \mathrm{x} \$$ Powder_Charge _ Database $!=$ "Unknown" $)$

xyplot ( $\mathrm{x}_{-}$nounknown $\$ \mathrm{FP}^{\sim} \mathrm{x}_{-}$nounknown $\$$ Powder_Charge _ Database $\mid \mathrm{x}_{-}$nounknown $\$$ Powd bwplot ( $\mathrm{x}_{-}$nounknown $\$ \mathrm{FP}^{\sim} \mathrm{x}_{-}$nounknown $\$$ Powder_Charge _ Database $\mid \mathrm{x}_{-}$nounknown $\$$ Powd \#fname <-paste0 (mainDir, "/ Plots", ".pdf")

\#write.table (i, fname, row.names $=$ FALSE)

png (file="FP_vs_DB_Charge_Weight_38Spl.png", width = 11, height = 8, units= xyplot $\left(\mathrm{x}_{-}\right.$nounknown $\$ \mathrm{FP}^{\sim} \mathrm{x}_{-}$nounknown $\$$ Powder_Charge _ Database $\mid \mathrm{x}_{-}$nounknown $\$$ Powd $\operatorname{dev}$. off ()

png ( fille="FP_vs_DB_Charg_Weight_357Mag_Box.png", width = 11, height $=8, u$ bwplot ( $x_{-}$nounknown $\$ F^{\sim} \mathrm{x}_{-}$nounknown $\$$ Powder_Charge _ Database $\mid \mathrm{x}_{-}$nounknown $\$$ Powd dev. off ()

\#—BF vs. DB CHG. WT. Given Sample CHG. WT.

i $<-" 38$ Special"

i $<-" 357$ Magnum"

rownames $<-$ a\$ Caliber_Ammo_Sample

start - with $M<-$ rownames $\%$ in $\%$ grep $(i$, rownames, value $=$ TRUE)

$\mathrm{x}<-\operatorname{subset}\left(\mathrm{a}, \quad\right.$ start - with $\_$) 
Powder . charge_DB $<-$ paste0 $(x \$$ Powder_Sample, "-" , x\$Powder_Charge_Sample) $\mathrm{x}<-\operatorname{cbind}\left(\mathrm{x}\right.$, Powder . charge $\left.\_\mathrm{DB}\right)$

$\#$ plot $\left(B F_{-} x, F P_{-} x, \operatorname{main}=i, \quad x l a b=" B F "\right.$, ylab $=" F P "$, col $=$ ifelse $(x[, 20$

$\mathrm{x}_{-}$nounknown $<-\operatorname{subset}(\mathrm{x}, \mathrm{x} \$$ Powder_Charge _ Database $!=$ "Unknown")

xyplot ( $x_{-}$nounknown $\$ B F^{\sim} x_{-}$nounknown $\$$ Powder_Charge_Database $\mid x_{-}$nounknown\$Powd bwplot (x_nounknown $\$ \mathrm{BF}^{\sim} \mathrm{x}_{-}$nounknown $\$$ Powder_Charge_Database $\mid \mathrm{x}_{-}$nounknown $\$$ Powd \#fname <- paste0 (mainDir, "/ Plots", ".pdf")

$\#$ write.table $(i$, fname, row.names $=$ FALSE)

png ( fille="BF_vs_DB_Charge_Weight_38Spl.png", width = 11, height = 8, units xyplot ( $x_{-}$nounknown $\$ B F^{\sim} x_{-}$nounknown $\$$ Powder_Charge_Database $\mid x_{-}$nounknown $\$$ Powd dev. off ()

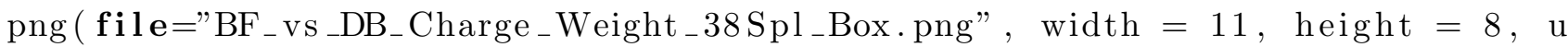
bwplot ( $\mathrm{x}_{-}$nounknown $\$ \mathrm{BF}^{\sim} \mathrm{x}_{-}$nounknown $\$$ Powder_Charge _Database $\mid \mathrm{x}_{-}$nounknown $\$$ Powd dev. off ()

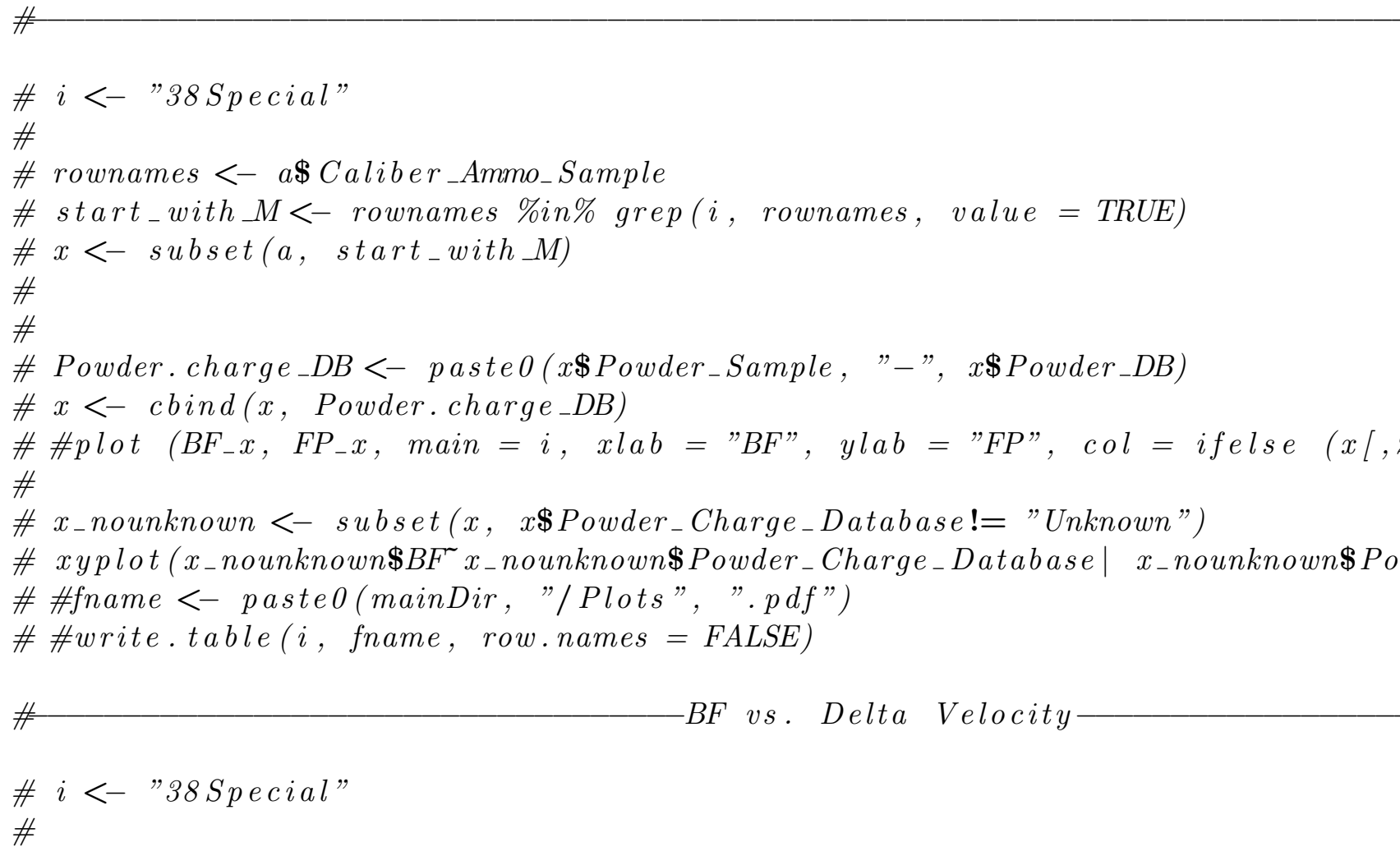




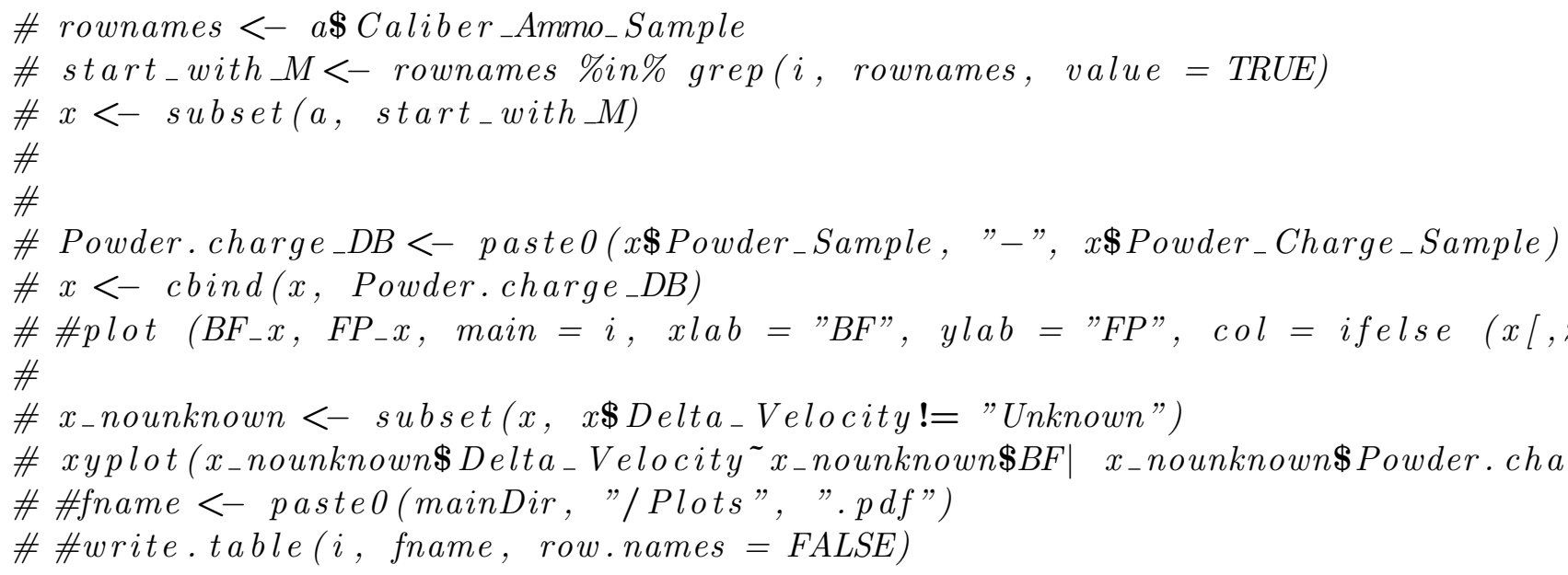

rownames <- a\$Caliber_Ammo_Sample start - with $\mathrm{M}<-$ rownames $\%$ in $\% \operatorname{grep}(\mathrm{i}$, rownames, value $=$ TRUE) $\mathrm{x}<-\operatorname{subset}(\mathrm{a}, \quad$ start - with $\mathrm{M})$ 
Powder . charge_DB $<-$ paste0 (x\$Powder_Sample, "-" , x\$Powder_Charge_Sample) $\mathrm{x}<-\operatorname{cbind}\left(\mathrm{x}\right.$, Powder . charge $\left.\_\mathrm{DB}\right)$

$\#$ plot $\left(B F_{-} x, F P_{-} x, \operatorname{main}=i, \quad x l a b=" B F "\right.$, ylab $=" F P "$, col $=$ ifelse $(x[, 20$

$\mathrm{x}_{-}$nounknown $<-\operatorname{subset}(\mathrm{x}, \mathrm{x} \$$ Delta_Pressure $!=$ "Unknown")

xyplot (x_nounknown $\$$ Delta_Pressure $\tilde{x}_{-}$nounknown $\$ B F \mid x_{-}$nounknown\$Powder. charg \#fname <- paste0(mainDir, "/ Plots", ".pdf")

\#write.table ( $i$, fname, row.names $=F A L S E)$

png (file=" Delta_ Pressure_vs_BF_357Mag.png", width = 11, height = 8, units=" xyplot ( $x_{-}$nounknown $\$$ Delta_Pressure $\tilde{x}_{-}$nounknown $\$ B F \mid x_{-}$nounknown $\$$ Powder. charg dev. off ()

\#—Delta Pressure vs. FP-

i $<-" 38$ Special"

i $<-" 357$ Magnum"

rownames <- a\$Caliber_Ammo_Sample

start with $_{-} \mathrm{M}<-$ rownames $\%$ in\% grep(i , rownames, value $=$ TRUE)

$\mathrm{x}<-\operatorname{subset}\left(\mathrm{a}\right.$, start with $\left._{\mathrm{M}}\right)$

Powder . charge_DB $<-$ paste0 (x\$Powder_Sample, "-" , x\$Powder_Charge_Sample)

$\mathrm{x}<-\operatorname{cbind}\left(\mathrm{x}\right.$, Powder . charge $\left.\_\mathrm{DB}\right)$

$\# p l o t\left(B F_{-} x, F P_{-} x\right.$, main $=i$, xlab $=" B F "$, ylab $=" F P "$, col $=$ ifelse $(x[, 20$

$x_{-}$nounknown $<-\operatorname{subset}(\mathrm{x}, \mathrm{x} \$$ Delta_Pressure $!=$ "Unknown" $)$

xyplot (x_nounknown\$Delta_Pressure $\tilde{x}_{-}$_nounknown $\$ F P \mid x_{-}$nounknown $\$$ Powder.charg \#fname <- paste0 (mainDir, "/Plots", ".pdf")

$\#$ write.table (i, fname, row.names $=$ FALSE)

png ( fille=" Delta_Pressure_vs_FP_357Mag.png", width = 11, height = 8, units= xyplot ( $x_{-}$nounknown $\$$ Delta_Pressure ${ }^{\sim} x_{-}$nounknown $\$ F P \mid \quad x_{-}$nounknown $\$$ Powder. charg $\operatorname{dev}$. off ()

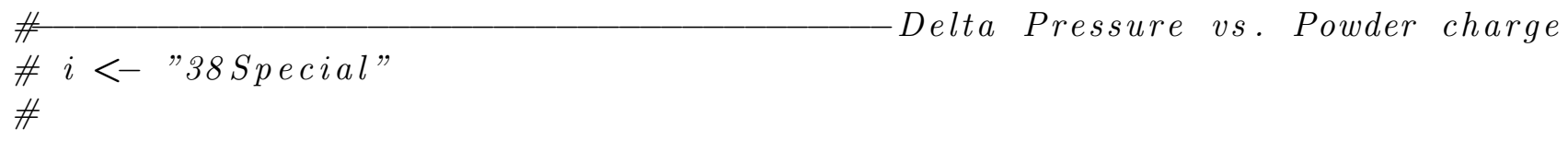




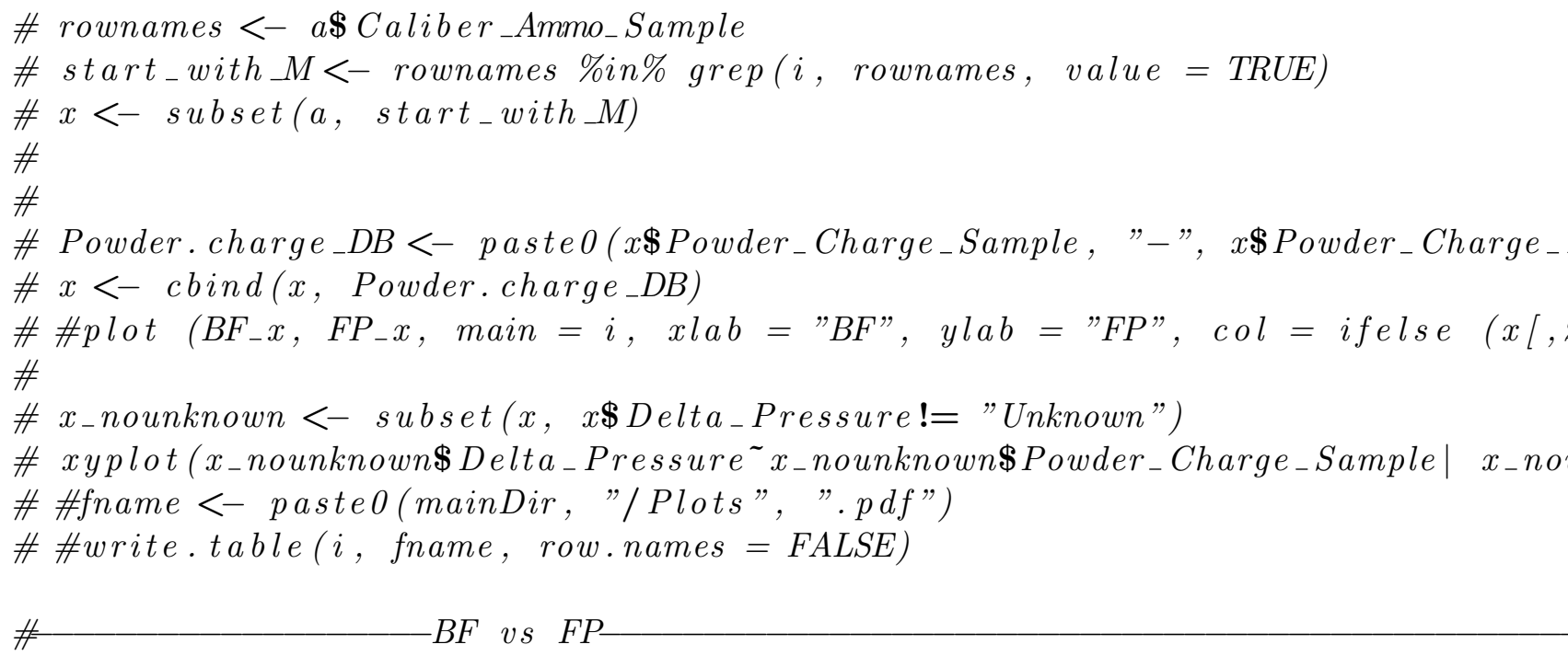

rownames $<-$ a\$Caliber_Ammo_Sample

start with $_{\mathrm{M}}<-$ rownames $\%$ in $\% \operatorname{grep}(\mathrm{i}$, rownames, value $=$ TRUE)

$\mathrm{x}<-\operatorname{subset}(\mathrm{a}$, start - with $\mathrm{M})$

Powder . charge_DB $<-$ paste0 (x\$Powder_Charge_Sample, "-" , x\$Powder_Charge_Da $\mathrm{x}<-\operatorname{cbind}(\mathrm{x}$, Powder . charge $\mathrm{DB})$

\#plot $\left(B F_{-} x, F_{-} x, \operatorname{main}=i, \quad x l a b=" B F ", y l a b=" F P "\right.$, col $=$ ifelse $(x[, 20$

$\mathrm{x}_{-}$nounknown $<-\operatorname{subset}(\mathrm{x}, \mathrm{x} \$$ Delta_Pressure $!=$ "Unknown" $)$

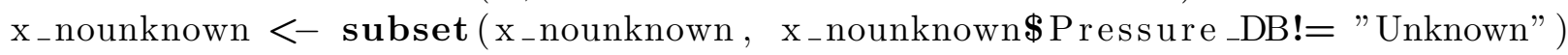
xyplot ( $\mathrm{x}_{-}$nounknown $\$ \mathrm{BF}^{\sim} \mathrm{x}_{-}$nounknown $\$ \mathrm{FP} \mid \mathrm{x}_{-}$nounknown $\$$ Powder . charge_DB, groups \#fname <- paste0 (mainDir, "/Plots", ".pdf")

$\#$ \#rite.table $(i$, fname, row.names $=$ FALSE)

png $\left(\mathbf{f i l e}=" \mathrm{BF}_{-}\right.$vs_FP_38Spl.png", width $=11$, height $=8$, units="in", res= 12 xyplot ( $\mathrm{x}_{-}$nounknown $\$ \mathrm{BF}^{\sim} \mathrm{x}_{-}$nounknown $\$ \mathrm{FP} \mid \mathrm{x}_{-}$nounknown$\$$ Powder. charge_DB, groups $\operatorname{dev}$. off () 